\title{
On the Resolvent of Differential Operators on Conic Manifolds
}

\author{
PAUL LOYA
}

\begin{abstract}
We describe the structure of the resolvent kernel of an elliptic cone (or Fuchs type) differential operator and give a precise description of the asymptotics of the kernel as the spectral parameter tends to infinity. The structure of the resolvent is investigated through a class of parameter-dependent pseudodifferential operators that incorporate the particular degeneracies of cone operators and their resolvents.
\end{abstract}

\section{Introduction.}

\subsection{Parameter-Dependent Operators.}

In this paper, we describe the structure of the resolvent of a cone differential operator acting between weighted Sobolev spaces on a compact manifold with boundary $X$, including a precise description of its asymptotics as the spectral parameter tends to infinity. Here, an $m$-th order cone differential operator is an operator $A \in x^{-m} \operatorname{Diff}_{b}^{m}(X)$, where $x$ is a boundary defining function on $X$ and where $\operatorname{Diff}_{b}^{m}(X)$ is the space of $m$-th order totally characteristic (or $b$-) differential operators. Thus, $A=x^{-m} P$, where on some collar neighborhood of $Y:=\partial X$,

$$
P=\sum_{k=0}^{m} P_{m-k}(x)\left(x D_{x}\right)^{k}, \quad D_{x}=\frac{1}{i} \partial_{x},
$$

where $P_{m-k}(x)$ is a smooth family of differential operators of order $m-k$ on $Y$. If $\Lambda$ denotes the spectral parameter domain, then under certain conditions on $A$ (called "full-ellipticity", see Section 1.3), we show that the resolvent $(A-\lambda)^{-1}$ exists on weighted Sobolev spaces and that its Schwartz kernel can be realized as a polyhomogeneous distribution on a "blown-up" manifold constructed from $\Lambda \times X^{2}$. The exponents of the polyhomogeneous expansions at the boundary faces of the blown-up manifold are given explicitly in terms of the boundary spectrum of $A$. In order to capture the precise 
asymptotics of the resolvent kernel, we define a class of cone pseudodifferential operators that depend on the spectral parameter $\lambda$ in a certain way. We then show that the resolvent is in this calculus. We will describe this calculus in Section 1.4. Throughout this paper, we confine ourselves to operators acting on functions in order to simplify the presentation; however, all the results in this paper have extensions to operators acting between sections of vector bundles.

The development of parameter-dependent pseudodifferential operators has a long history. Following Agmon's ideas $[1,2]$ in treating $\lambda$ as a covariable, a systematic formulation of such operators was initiated by Seeley [44] to investigate the structure of the complex powers of differential operators on manifolds without boundary. Closely related operators can be found in Gilkey [17], McOwen [32], Shubin [45], and undoubtedly others. Parameterdependent operators have been developed in other contexts. For instance, Gil [15] for cone differential operators, Grubb and Seeley [18, 19] and Rempel and Schulze [39] for boundary value problems, and Schrohe [41] for certain types of noncompact manifolds. The work of Gil is the closest to that of this paper, but it uses a very different methodology. It relies on techniques from the "edge theory" initiated by Schulze for the study of operators on manifolds with edge singularities, while our methods are based on the geometric "blow-up" techniques of Melrose. Further developments in the edge theory can be found in [31, 43], and a comparison of the methods of Schulze and Melrose can be found in [25].

There are two central reasons for defining parameter-dependent pseudodifferential operators. The first reason is that these operators provide a framework that allows one to understand the precise structure of operators defined using the classical functional calculus [40, 13, 12]. For example, using parameter-dependent operators, one can show that the complex powers of differential operators are entire families of pseudodifferential operators. See [44] for differential operators on closed manifolds, [41] on certain noncompact manifolds, $[39,18]$ on manifolds with boundary, and [27] on conic manifolds. The second, and perhaps the most important reason to study parameter-dependent operators is to understand the trace expansion of the resolvent: $\operatorname{Tr}(A-\lambda)^{-N}$ as the spectral parameter $\lambda$ tends to infinity. Here, $N$ is taken large enough to ensure that $(A-\lambda)^{-N}$ is of trace class. As the resolvent and heat operators are related by the Laplace and inverse Laplace transforms, the trace asymptotics of the resolvent are directly related to the small time heat trace asymptotics. The heat trace asymptotics generate many applications in noncommutative geometry, spectral asymptotics, and index theory to name a few, cf. Gilkey's book [17], especially the references 
in Ch. 5. In Section 1.2, we will discuss more on trace expansions.

Besides the work of Gil [15] already mentioned, other related works include that of Brüning and Seeley [6, 4], Cheeger [8], Lesch [26], and Mooers [38]. However, these authors analyze resolvents and heat operators without the formal development of parameter-dependent operators. Cheeger was the first to analyze the heat operator of the Laplacian on a conic manifold. The methods of Mooers are the closest to those of this paper: she also uses blow-up techniques, cf. [35, Ch. 7], to construct the Schwartz kernel of the heat operator for the cone Laplacian as a polyhomogeneous function on a blown-up manifold.

As an application of the methods of this paper, we show that the complex powers of a cone operator define an entire family of $b$-pseudodifferential operators [27]. In [28], we analyze the small time heat trace asymptotics of cone operators. We also attain (principle) asymptotic estimates for the eigenvalue counting function and the spectral function for arbitrary order self-adjoint cone operators. A possible future application of this paper includes sharpening these asymptotic formulas following Agmon and Kannai [2], Hörmander [20], Grubb [18], among others. The asymptotic formulas in [28] generalize those for the cone Laplacian obtained by Ivriu [21], Kalka and Ménikoff [22], and Phạm The Lại and Petkov [24]. Finally, in [16], we identify the noncommutative residues for pseudodifferential operators on conic manifolds, cf. [36, 42], via heat traces.

We note that in this paper, we focus on parabolic techniques for cone operators. See for instance, Cheeger and Taylor [10] and Melrose and Wunsch [37], for treatments of hyperbolic methods.

\subsection{Trace Expansions.}

Let $B \in x^{-\beta} \operatorname{Diff}_{b}^{m^{\prime}}(X)$, where $\beta \in \mathbb{R}, m^{\prime} \in \mathbb{N}_{0}$. Then given a fully elliptic cone differential operator $A$ of order $m$ (see Section 1.3), we will prove the following trace expansion, see Theorem 8.4:

Full Trace Expansion. If $m^{\prime}-m N<-n$ where $n=\operatorname{dim} X$ and if $\beta<m$, then $B(A-\lambda)^{-N}$ is trace class, and as $\lambda \rightarrow \infty$ in $\Lambda$, we have

$$
\operatorname{Tr} B(A-\lambda)^{-N} \sim \sum_{k=0}^{\infty} a_{k} \lambda^{\frac{m^{\prime}+n-k}{m}-N}+\sum_{k=0}^{\infty}\left\{b_{k} \log \lambda+c_{k}\right\} \lambda^{\frac{\beta-k}{m}-N},
$$

where $b_{k}=0$ unless $k \in \beta-m^{\prime}-n+\mathbb{N}_{0}$.

Once the asymptotics of the kernel of $B(A-\lambda)^{-N}$ are understood, this trace expansion follows from Melrose's pushforward theorem (see Lemma 1.1 
in the Appendix). If $\Lambda$ is the complement of a sector in the right-half plane, then (1.2) implies the following heat trace expansion: As $t \downarrow 0$,

$$
\operatorname{Tr} B e^{-t A} \sim \sum_{k=0}^{\infty} \alpha_{k} t^{\frac{k-m^{\prime}-n}{m}}+\sum_{k=0}^{\infty}\left\{\beta_{k} \log t+\gamma_{k}\right\} t^{\frac{k-\beta}{m}} .
$$

In [28], we give a formula for the constant term, and explicit local formulas for all the singular and log coefficients in the expansion (1.3). In joint work with Gil [16], we treat the case when $B$ is an arbitrary order cone pseudodifferential operator. In this case, the expansion contains $\log ^{2}$ terms, and using the techniques and results of [28], we give explicit formulas for all the log and $\log ^{2}$ coefficients in terms of residue trace functionals.

With $B=\mathrm{Id}$, the heat trace expansion has been proved in various contexts. In particular, Gil [15] was the first to prove such a heat trace expansion for arbitrary order fully elliptic cone operators. For second order operators, Brüning and Seeley [6, 4], Callias [7], Cheeger [8, 9], Chou [11], and Mooers [38] also obtain a full heat trace expansion. Cheeger was the first to obtain a full trace expansion for the cone Laplacian. Brüning and Seeley obtain the expansion for second order regular singular operators using the "Singular Asymptotics Lemma" of [5]. Lesch [26] generalizes the techniques of Brüning and Seeley to obtain the heat trace expansion for arbitrary order self-adjoint, constant coefficient cone operators; but in this case, all the $\log$ terms for $k>0$ vanish. Finally, under ellipticity conditions similar to ours, $\mathrm{Karol}^{\prime}$ [23] obtains the heat expansion for arbitrary even order cone operators.

\subsection{Full-Ellipticity.}

Let $\Lambda \subseteq \mathbb{C}$ be a closed angle, $\alpha \in \mathbb{R}$, and let $A$ be an $m$-th order cone differential operator. We review a condition introduced by Gil [15] that ensures the invertibility of $A-\lambda$ on weighted Sobolev spaces. Let $A=x^{-m} P$ where $P$ is an $m$-th order $b$-differential operator. Now for $\lambda$ sufficiently large in $\Lambda$, we want to determine when we can invert

$$
A-\lambda=x^{-m}\left(P-x^{m} \lambda\right): x^{\alpha} H_{b}^{p}(X) \longrightarrow x^{\alpha-m} H_{b}^{p-m}(X),
$$

for any $p \in \mathbb{R}$, where $H_{b}^{*}$ denotes the $b$-Sobolev spaces. There are three "degeneracies" of (1.4): 1) $\lambda$ near infinity with $x$ bounded away from zero; 2) $x=0$ with $\lambda$ bounded away from infinity; and 3) $x=0$ and $\lambda=\infty$.

Consider $\lambda$ near infinity with $x$ bounded away from zero. Then disregarding the $x$ factors, our first requirment is that the parameter-dependent 
symbol of $P-\lambda$ be invertible. That is, if ${ }^{b} \sigma_{m}(P)(\xi)$ represents the totally characteristic (or $b$-) principal symbol of $P$, see [35], then we require that

$$
{ }^{b} \sigma_{m}(P)(\xi)-\lambda \text { be invertible for all } \xi \neq 0 \text { and } \lambda \in \Lambda \text {. }
$$

Here, $\xi$ is an element of the $b$-cotangent bundle. In fact, we require that the condition (1.5) hold up to and including the boundary $Y$ of $X$.

We now deal with the singularity at $x=0$ with $\lambda$ bounded away from infinity. Then disregarding the term $x^{m} \lambda$, we need to handle the boundary degeneracy of $x^{-m} P$. To do so, write $P$ as in (1.1), and define $I(A):=$ $s^{-m} \sum_{k=0}^{m} P_{m-k}(0)\left(s D_{s}\right)^{k}$ for $s \in[0, \infty)$. Then $I(A)$ models the behavior of $A$ near $x=0$. Our second requirement is that

$$
I(A): s^{\alpha} H_{b}^{p}\left(Y^{\wedge}\right) \longrightarrow s^{\alpha-m} H_{b}^{p-m}\left(Y^{\wedge}\right)
$$

be invertible, where $Y^{\wedge}:=[0, \infty) \times Y$. Note that (1.5) and (1.6) imply that $A-\lambda$ in (1.4) is Fredholm for all $\lambda \in \Lambda$ (see Theorem 3.2). Taking the Mellin transform of $s^{m} I(A)$ in $s$, our second condition can be stated in terms of the invertibility of the conormal symbol (or normal operator) of $P$.

To ensure that $A-\lambda$ is invertible for $\lambda$ sufficiently large, we need to deal with our third and final singularity: $x=0, \lambda=\infty$. To do so, we introduce "blow-ups". Let $\lambda=r^{-m} a(r, \omega) \omega$, where $\omega=\lambda /|\lambda|$ and where $a(r, \omega)>0$ is a smooth positive function of $r \in[0, \infty)$ and $\omega$ (smooth and positive even down to $r=0$ ). Then $\lambda=\infty$ corresponds to $r=0$. We now blow-up $x=0$, $r=0$; that is, we introduce polar coordinates at $x=0, r=0$. Convenient coordinates to work with are projective coordinates. Thus, consider the coordinates $r, s=x / r$. Then $x=s r$ and $x D_{x}=s D_{s}$. Hence,

$$
\begin{aligned}
A-\lambda & =s^{-m} r^{-m} \sum_{k=0}^{m} P_{m-k}(s r)\left(s D_{s}\right)^{k}-r^{-m} a(r, \omega) \omega \\
& =r^{-m}\left\{s^{-m} \sum_{k=0}^{m} P_{m-k}(s r)\left(s D_{s}\right)^{k}-a(r, \omega) \omega\right\} .
\end{aligned}
$$

Note that $r=0$ in $(r, s)$ coordinates corresponds to $x=0, r=0$ in the original coordinates. Thus, as $a(r, \omega)>0,(1.7)$ suggests our third requirement for full-ellipticity:

$$
I(A)-\lambda \text { be invertible for all } \lambda \in \Lambda \text { sufficiently large. }
$$

Invertibility will be on certain "cone" Sobolev spaces, see Section 6 .

An operator $A$ is fully elliptic with respect to $\alpha$ on $\Lambda$ if the three "symbols" of $A-\lambda$ are invertible in the sense that conditions (1.5), (1.6), and (1.8) are satisfied. 


\subsection{Statement of Results and Outline of Paper.}

In Sections 2 and 3, we review some aspects of analysis on manifolds with corners, including asymptotic expansions, blow-ups, and $b$-pseudodifferential operators. We also review the Fredholm properties of cone differential operators. In Sections 4 and 5, we introduce our parameter-dependent spaces that allow $A-\lambda$ to be inverted for $A$ fully elliptic. We now describe these spaces by explaining how we plan to invert $A-\lambda$.

First, writing $A-\lambda=x^{-m}\left(P-x^{m} \lambda\right)$, we want to invert (that is, find a parametrix for) the "b-part": $P-x^{m} \lambda$. Observe that $x$ and $\lambda$ are coupled into the one parameter $x^{m} \lambda$. In Section 4, we consider $b$ pseudodifferential operators which have local symbols that depend on the "parameter" $\mu:=x^{m} \lambda$. Thus, roughly speaking, we consider parameterdependent $b$-pseudodifferential operators $B(\lambda)$ such that if $\mathcal{U}$ is a coordinate patch on $X$, then the local totally characteristic (or $b$-) symbol of $B(\lambda)$ is of the form $b\left(x^{m} \lambda, w, \xi\right)$ where $w=\left(w_{1}, \ldots, w_{n}\right)$ are the coordinates on $\mathcal{U}$. We call this space of operators the "small calculus" of tempered operators. We use the word "tempered" because we require that the local symbols $b(\mu, w, \xi)$ satisfy certain growth conditions in $\mu$ and $\xi$. We also discuss the mapping and composition properties of the small calculus. The proofs of the mapping and composition properties (Proposition 4.2, Lemma 4.3, and Theorem 4.4) are written in careful detail for those readers interested in how $b$-operators are manipulated. Unfortunately, they are quite technical as they involve analyzing pushforwards and pullbacks of conormal distributions on blown-up manifolds. The proofs may be skipped over at a first reading.

Second, we invert $A \approx I(A)$ near the boundary. This can be done within the usual calculus of $b$-pseudodifferential operators.

Finally, we want to invert the operator $I(A)-\lambda$ found in (1.8). In order to do this we introduce a blown-up manifold $\mathcal{T}$ connected to the arguments used to derive condition (1.8). Let $x$ and $x^{\prime}$ denote the boundary defining functions for the left and right factors of $X^{2}$. The first step is to blowup $x=x^{\prime}=0$ in $X^{2}$, which constructs the manifold $X_{b}^{2}$, the $b$-stretched product. Let $\rho$ be a boundary defining function for the blown-up face in $X_{b}^{2}$ (e.g. $\rho=x+x^{\prime}$ is such a function). The second step is to blow-up $\rho=0$, $r=0$, where for $\lambda$ large, $\lambda \sim r^{-m}$ for $r$ near 0 . This creates the manifold with corners $\mathcal{T}$. In Section 5, we define a space of parameter-dependent operators whose Schwartz kernels are polyhomogeneous functions on the blown-up manifold $\mathcal{T}$.

The "full calculus" of tempered operators consists of a sum of parameter dependent operators: $B(\lambda)+C(\lambda)+D(\lambda)$ where $B(\lambda)$ is in the small calculus 
of tempered operators, where $C(\lambda)$ is described in the previous paragraph, and where $D(\lambda)$ is a parameter-dependent operator whose Schwartz kernel is a polyhomogeneous function on $\Lambda \times X^{2}$, vanishing to infinite order at $\lambda=\infty$. In Section 5, we discuss the mapping and composition properties of the full calculus.

In Section 6, we prove the main result of this paper (see Theorem 6.1): For $\lambda$ sufficiently large, the resolvent $(A-\lambda)^{-1}$ exists and is an element of the full calculus of tempered operators. We emphasize that the composition properties of our calculus of parameter-dependent operators make the construction of $(A-\lambda)^{-1}$ very similar to the construction of a parametrix for an elliptic (non parameter-dependent) $b$-differential operator.

In Section 7, we show precisely how the singularities of the Schwartz kernel of $(A-\lambda)^{-1}$ accumulate near the diagonal as $\lambda \rightarrow \infty$. This is expressed by showing that the kernel of $(A-\lambda)^{-1}$ can be realized as a polyhomogeneous distribution on a blown-up manifold constructed from $\Lambda \times X^{2}$. The exponents of the polyhomogeneous expansions at the boundary faces of the blown-up manifold are given in terms of the boundary spectrum of $A$.

In Section 8, we prove the trace expansion (1.2), and finally, in the Appendix we collect various results about asymptotic expansions and about $b$-pseudodifferential operators.

In conclusion, I thank Juan Gil and the referee for helpful comments in improving this paper.

\section{Manifolds with corners.}

The main references for this section are [14] and [35]. Other references are $[29]$ and [30]. We define $\mathbb{N}_{0}:=\{0,1,2, \ldots\}$ and $\mathbb{N}:=\{1,2, \ldots\}$.

\subsection{Manifolds with corners and conormal functions.}

An $n$ dimensional manifold with corners $X$ is a paracompact topological space with local models of the form $\mathbb{R}^{n, k}:=[0, \infty)_{x} \times \mathbb{R}_{y}^{n-k}$, where $k$ can run between 0 and $n$, such that $X$ has only finitely many boundary hypersurfaces, say $\left\{H_{1}, \ldots, H_{r}\right\}$ for some $r \in \mathbb{N}_{0}$, where each $H_{i}$ is imbedded. In our applications, the main source of manifolds with corners will come from the process of blow-up (see Section 2.2). By definition, it follows that each boundary hypersurface $H_{i}$ is itself a manifold with corners, and that if $X$ is compact, then near $H_{i}$ there exists a local diffeomorphism $X \cong[0,1) \times H_{i}$. The set of boundary hypersurfaces is denoted by $M_{1}(X)$. A total boundary defining function is a function of the form $\rho=\prod_{i=1}^{r} \rho_{i}$, where $\rho_{i}$ is a 
boundary defining function for $H_{i}$.

The $b$-density bundle, $\Omega_{b}$, is the trivial line bundle with sections of the form $\rho^{-1} \mu$, where $\mu$ is a smooth density on $X$, and where $\rho$ is a total boundary defining function on $X$.

From now on, $X$ will always be compact. The space of $b$-differential operators of order $m$, Diff $b_{b}^{m}(X)$, is the space of operators $P$ on $C^{\infty}(X)$, such that locally on a patch $\mathbb{R}^{n, k}=[0, \infty)_{x}^{k} \times \mathbb{R}_{y}^{n-k}$, we can write

$$
P=\sum_{|\alpha|+|\beta| \leq m} a_{\alpha, \beta}(x, y)\left(x D_{x}\right)^{\alpha} D_{y}^{\beta}, \quad \text { where } a_{\alpha, \beta}(x, y) \in C^{\infty}\left(\mathbb{R}^{n, k}\right) .
$$

We now define various classes of conormal functions. A multi-index $\alpha$ on $X$ is a map $\alpha: M_{1}(X) \rightarrow \mathbb{R}$, or equivalently, an $r$-tuple $\left(\alpha_{1}, \ldots, \alpha_{r}\right)$ of real numbers. Let $\rho$ be a total boundary defining function for $X$ and $\alpha$ a multi-index. Then the space of symbols of order $\alpha, S^{\alpha}(X)$, consists of functions $u$ such that $\operatorname{Diff}_{b}^{*}(X) u \subseteq \rho^{-\alpha} L^{\infty}(X)$, where $\rho^{-\alpha}:=\prod_{i=1}^{r} \rho_{i}^{-\alpha_{i}}$. The Sobolev lemma implies that symbols are smooth on the interior of $X$; they may however, have singularities at $\partial X$. For example, given $a \in \mathbb{C}$ and $k \in \mathbb{N}_{0}, x^{a}(\log x)^{k} \in S^{\alpha}([0,1))$ for all $\alpha>-\operatorname{Re} a$.

An index set (or $C^{\infty}$ index set) is a discrete subset $E \subseteq \mathbb{C} \times \mathbb{N}_{0}$ satisfying the following conditions: if $(z, k) \in E$, then $(z+\ell, j) \in E$ for all $\ell \in \mathbb{N}_{0}$ and $0 \leq j \leq k$, and given any $N \in \mathbb{N}_{0},\{(z, k) \in E \mid \operatorname{Re} z \leq N\}$ is a finite set.

Given a multi-index $\alpha$ and a hypersurface $H$, we define a multi-index $\alpha_{H}$ on $H$ as follows. If $G \in M_{1}(H)$, then $G=H \cap H^{\prime}$ for some $H^{\prime} \in M_{1}(X)$. We define $\alpha_{H}(G):=\alpha\left(H^{\prime}\right)$. Let $E$ be an index set. A function $u \in S^{\alpha}(X)$ (for some $\alpha$ ) is said to have an asymptotic (or classical) expansion at $H$ with index set $E$, if given any product decomposition $X \cong[0,1)_{x} \times H_{y}$ of $X$ near $H$, for each $(z, k) \in E$, there exists a $u_{(z, k)} \in S^{\alpha_{H}}(H)$ such that for each $N \in \mathbb{N}$, there exists an $M \in \mathbb{N}$ with

$$
u(x, y)-\sum_{(z, k) \in E, \operatorname{Re} z \leq M} x^{z}(\log x)^{k} u_{(z, k)}(y) \in x^{N} S^{\left(0, \alpha_{H}\right)}([0,1) \times H) .
$$

We then write $u \sim \sum_{(z, k) \in E} x^{z}(\log x)^{k} u_{(z, k)}$. Thus, an asymptotic expansion is just a generalized "Taylor expansion". In the appendix of [29], it is shown that the expansion (2.1) is defined independent of the choice of product decomposition of $X$ near $H$. Note that if $E=\varnothing$, then (2.1) holds for all $N$ if and only if $u$ vanishes to infinite order at $H$. Also, if $E=\mathbb{N}_{0}$, then (2.1) holds for all $N$ if and only if $u$ is smooth up to $H$.

An index family associated to a subset $\mathcal{A} \subseteq M_{1}(X)$ is a set $\mathcal{E}=\left\{E_{H} \mid H \in\right.$ $\mathcal{A}\}$, where each $E_{H}$ is an index set. We define $\mathcal{A}^{\mathcal{E}}(X)$ to be the space of 

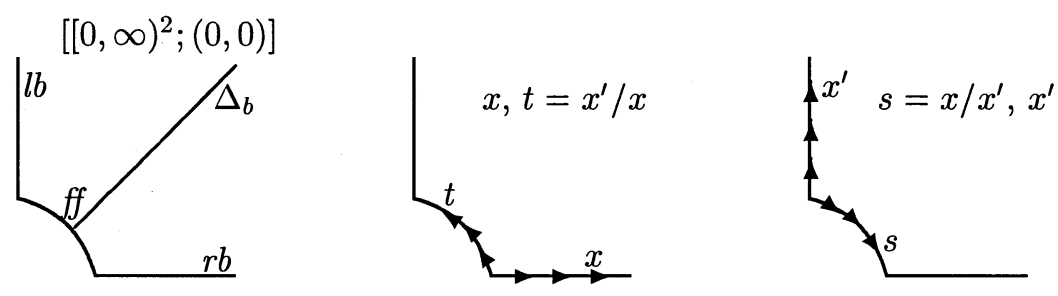

Figure 1: The manifold $\left[[0, \infty)^{2} ;(0,0)\right]$, along with two sets of projective coordinates.

functions that have an expansion at each $H \in \mathcal{A}$ with index set $E_{H}$. If $\mathcal{A}=M_{1}(X)$, we denote $\mathcal{A}^{\mathcal{E}}(X)$ by $\mathcal{A}_{p h g}^{\mathcal{E}}(X)$.

\subsection{Blow-ups.}

Let $X=[0, \infty)_{x} \times[0, \infty)_{x^{\prime}}$ and $Y=(0,0)$. Then we define " $X$ blown-up at $Y$ " as the set $[X ; Y] \equiv[0, \infty)_{r} \times \mathbb{S}_{\theta}^{1,2}$, where $\mathbb{S}^{1,2}=\mathbb{S}^{1} \cap[0, \infty)^{2}$, and where $r=\left|\left(x, x^{\prime}\right)\right|$ and $\theta=\tan ^{-1}\left(x^{\prime} / x\right)$ are polar coordinates about $Y$. See Figure 1 for a picture of $[X ; Y]$. Thus, blow-up of $Y$ is just the introduction of polar coordinates about $Y$. The blow-down map $\beta:[X ; Y] \rightarrow X$ is by definition the usual polar coordinates map: $\beta(r, \theta)=(r \cos \theta, r \sin \theta)$. The left boundary, $l b$, is where $\theta=\pi / 2$, the right boundary, $r b$, is where $\theta=0$, and the front face, ff, is where $r=0$. The $b$-diagonal, $\Delta_{b}$, is the set where $\theta=\pi / 4$. Other useful coordinates are the coordinates $(x, z)$, where $z=\log \left(x / x^{\prime}\right)$, which give the decomposition:

$$
[X ; Y] \backslash\{l b, r b\} \cong[0, \infty)_{x} \times \mathbb{R}_{z}, \quad \text { where } \Delta_{b}=[0, \infty)_{x} \times\{0\}_{z} .
$$

Projective polar coordinates are also useful:

$$
[X ; Y] \backslash\{r b\} \cong[0, \infty)_{s} \times[0, \infty)_{x^{\prime}}, \text { where } s=x / x^{\prime} .
$$

Here, $l b, r b, f f$, and $\Delta_{b}$ are the sets where $s=0, s=+\infty, x^{\prime}=0$, and $s=1$ respectively. Reversing the roles of $x$ and $x^{\prime}$ gives another set of projective coordinates. See Figure 1 for a description of these coordinates.

In general, given a manifold with corners $X$ and a $p$ - (or imbedded) submanifold $Y$ of $X$, one can define " $X$ blown-up at $Y$ ", $[X ; Y]$, by taking polar coordinates about $Y$. The boundary face created in the blow-up is called the front face, denoted by $f f[X ; Y]$, and the polar coordinates map $\beta:[X ; Y] \rightarrow X$ is called the blow-down map. See [14] for a precise definition of the mathematical process of blow-up. If $Z \subseteq X$ is a closed subset of $X$, 
then we define the lift of $Z$ into $[X ; Y], \beta^{*} Z \subseteq[X ; Y]$, as $\beta^{*} Z:=\beta^{-1}(Z)$ if $Z \subseteq Y$; or as $\beta^{*} Z:=\overline{\beta^{-1}(Y \backslash Z)}$ if $Z=\overline{Z \backslash Y}$. For example, if $X=[0, \infty)^{2}$ and $Y=(0,0)$, then $l b, r b, f f$, and $\Delta_{b}$ shown in Figure 1 are the lifts of $\{0\} \times[0, \infty),[0, \infty) \times\{0\},(0,0)$, and $\left\{x=x^{\prime}\right\}$ respectively. If $\beta^{*} Z$ is defined and if in addition, $\beta^{*} Z$ is a p-submanifold of $[X ; Y]$, then $[X ; Y]$ blown-up along $\beta^{*} Z$ is defined, and we denote it by $[X ; Y ; Z] \equiv\left[[X ; Y] ; \beta^{*} Z\right]$.

Assume now that $X$ is a compact manifold with connected boundary $Y$. Then we define the $b$-stretched product, $X_{b}^{2}$, by $X_{b}^{2}:=\left[X^{2} ; Y \times Y\right]$. If $\beta: X_{b}^{2} \longrightarrow X^{2}$ is the blow-down map, we set $l b:=\beta^{*}(Y \times X), r b:=$ $\beta^{*}(X \times Y)$, and $f f:=\beta^{*}(Y \times Y)$. The $b$-diagonal is defined by $\Delta_{b}:=\beta^{*}(\Delta)$, where $\Delta$ is the diagonal in $X^{2}$. Locally, Figure 1 describes $X_{b}^{2}$. Indeed, let $\mathcal{U}=[0,1)_{x} \times \mathbb{R}_{y}^{n-1}$ be a coordinate patch on $X$ near $Y$. Then, $X^{2} \cong$ $[0,1)_{\left(x, x^{\prime}\right)}^{2} \times \mathbb{R}_{y}^{n-1} \times \mathbb{R}_{y^{\prime}}^{n-1}$, where $\left(x^{\prime}, y^{\prime}\right)$ are the coordinates on the right factor of $\mathcal{U}^{2}$. It follows that locally

$$
X_{b}^{2} \cong\left[[0,1)^{2} ;(0,0)\right] \times \mathbb{R}_{y}^{n-1} \times \mathbb{R}_{y^{\prime}}^{n-1} .
$$

By (2.2), coordinates on $X_{b}^{2}$ away from $l b$ and $r b$, are given by

$$
X_{b}^{2} \cong \mathcal{U} \times \mathbb{R}_{z}^{n}, \quad \text { where } z=\left(\log \left(x / x^{\prime}\right), y-y^{\prime}\right) .
$$

Observe that in these coordinates, $\Delta_{b} \cong \mathcal{U} \times\{0\}$.

\section{Calculi of pseudodifferential operators.}

\section{1. $b$-pseudodifferential operators.}

More on $b$-pseudodifferential operators can be found in the Appendix. For the rest of the paper, we will assume that $X$ is a compact manifold with connected boundary. We will denote $\partial X$ by $Y$.

Let $0<\nu \in C^{\infty}\left(X, \Omega_{b}\right)$ be any trivialization of $\Omega_{b}$ (see Section 2.1). We denote by $\nu^{\prime}$ the lift of $\nu$ to $X^{2}$ under the projection $X^{2} \ni\left(y, y^{\prime}\right) \mapsto y^{\prime} \in X$.

Definition 1. The space of b-pseudodifferential operators of order $m \in \mathbb{R}$, $\Psi_{b}^{m}(X)$, consists of operators $A$ on $C^{\infty}(X)$ that have a Schwartz kernel $K_{A}$ satisfying the following two conditions:

(1) Given $\varphi \in C_{c}^{\infty}\left(X_{b}^{2} \backslash \Delta_{b}\right)$, the kernel $\varphi K_{A}$ is of the form $k \nu^{\prime}$, where $k \in C^{\infty}\left(X_{b}^{2}\right)$ and vanishes in Taylor series at the sets $l b$ and $r b$.

(2) Given a coordinate patch of $X_{b}^{2}$ near $\Delta_{b}$ of the form $\mathcal{U}_{y} \times \mathbb{R}_{z}^{n}$ such that $\Delta_{b} \cong \mathcal{U} \times\{0\}$. (cf. (2.3)), and given $\varphi \in C_{c}^{\infty}\left(\mathcal{U} \times \mathbb{R}^{n}\right)$, we have 


$$
\varphi K_{A}=\int_{\mathbb{R}^{n}} e^{i z \cdot \xi} a(y, \xi) d \xi \cdot \nu^{\prime}, \quad d \xi=\frac{1}{(2 \pi)^{n}} d \xi
$$

where $a(y, \xi)$ is a classical symbol of order $m$.

Gluing together the principal symbols of the local operators (3.1) gives a (principal) symbol map, ${ }^{b} \sigma_{m}(A): \Psi_{b}^{m}(X) \longrightarrow C_{\mathrm{hom}(m)}^{\infty}\left({ }^{b} T^{*} X\right)$, where $C_{\mathrm{hom}(m)}^{\infty}\left({ }^{b} T^{*} X\right)$ is the space of smooth homogeneous functions of degree $m$ on ${ }^{b} T^{*} X \backslash\{0\}$, where ${ }^{b} T^{*} X$ is the $b$-cotangent bundle (see [35]). If ${ }^{b} \sigma_{m}(A)$ is invertible, then $A$ is called elliptic. The symbol map gives an exact sequence

$$
0 \hookrightarrow \Psi_{b}^{m-1}(X) \hookrightarrow \Psi_{b}^{m}(X) \stackrel{b_{\sigma_{m}}}{\longrightarrow} C_{\mathrm{hom}(m)}^{\infty}\left({ }^{b} T^{*} X\right) \rightarrow 0 .
$$

The space $L_{b}^{2}(X)$ consists of those functions which are square integrable with respect to $\nu$. For each $m \in \mathbb{R}$, the $b$-Sobolev space of order $m, H_{b}^{m}(X)$, is the space of distributions $u$ such that $\Psi_{b}^{m}(X) u \subseteq L_{b}^{2}(X)$. If $A \in \Psi_{b}^{m}(X)$, then $A: H_{b}^{s}(X) \longrightarrow H_{b}^{s-m}(X)$ continuously for any $s \in \mathbb{R}$.

Fix a boundary defining function $x$ on $X$ and assume that $X \cong[0,1)_{x} \times Y$ near $Y$. In the coordinate patch (2.3) on $X_{b}^{2}$, let $A \in \Psi_{b}^{m}(X)$ be given locally by (3.1) with $\nu^{\prime}=\left|\left(d x^{\prime} / x^{\prime}\right) d y^{\prime}\right|$. Let $\xi=\left(\tau, \xi^{\prime}\right)$ where $\xi^{\prime}=\left(\xi_{2}, \ldots, \xi_{n}\right)$. Then the normal operator of $A, \widehat{A}(\tau) \in \Psi^{m}(Y)$, is the operator defined locally by

$$
\widehat{A}(\tau):=\frac{1}{(2 \pi)^{n-1}} \int e^{i\left(y-y^{\prime}\right) \cdot \xi^{\prime}} a\left(0, y, \tau, \xi^{\prime}\right) d \xi^{\prime} \cdot\left|d y^{\prime}\right| .
$$

For example, if $P=\sum_{k=0}^{m} P_{m-k}(x)\left(x D_{x}\right)^{k}$, where $P_{m-k}(x)$ is a smooth family of differential operators of order $m-k$ on $Y$ and $D_{x}=\frac{1}{i} \partial_{x}$, then

$$
\widehat{P}(\tau)=\sum_{k=0}^{m} P_{m-k}(0) \tau^{k}: H^{s}(Y) \longrightarrow H^{s-m}(Y) .
$$

Recall that given a smooth $b$-density $0<\nu \in C^{\infty}\left(X, \Omega_{b}\right), \nu^{\prime}$ denotes the lift of $\nu$ to $X^{2}$ under the projection $X^{2} \ni\left(y, y^{\prime}\right) \mapsto y^{\prime} \in X$. If $\mathcal{E}=\left(E_{l b}, E_{r b}\right)$ is an index family on $X^{2}$, then $\Psi^{-\infty, \mathcal{E}}(X)$ consists of those operators with integral kernels of the form $k \nu^{\prime}$ where $k \in \mathcal{A}_{\text {phg }}^{\mathcal{E}}\left(X^{2}\right)$. Here, the space of polyhomogeneous functions " $\mathcal{A}_{p h g}$ " is defined at the end of Section 2.1. Thus,

$$
A \in \Psi^{-\infty, \mathcal{E}}(X) \quad \Longleftrightarrow \quad K_{A}=k \nu^{\prime}, \quad k \in \mathcal{A}_{p h g}^{\mathcal{E}}\left(X^{2}\right) .
$$


Since the kernels of operators in $\Psi^{-\infty, \mathcal{E}}(X)$ are polyhomogeneous functions on $X^{2}$, it follow that for $\alpha \in \mathbb{R}$ with $E_{r b}>-\alpha$, any operator $A \in \Psi^{-\infty, \mathcal{E}}(X)$ defines a continuous linear map

$$
A: x^{\alpha} H_{b}^{s}(X) \longrightarrow \mathcal{A}_{p h g}^{E_{l b}}(X), \quad \text { for any } s \in \mathbb{R}
$$

If $\mathcal{E}=\left(E_{l b}, E_{r b}, E_{f f}\right)$ is an index family on $X_{b}^{2}$, then we define

$$
A \in \Psi_{b}^{-\infty, \mathcal{E}}(X) \quad \Longleftrightarrow \quad K_{A}=k \nu^{\prime}, \quad k \in \mathcal{A}_{p h g}^{\mathcal{E}}\left(X_{b}^{2}\right),
$$

and the full calculus is the sum:

$$
\widetilde{\Psi}_{b}^{m, \mathcal{E}}(X):=\Psi_{b}^{m}(X)+\Psi_{b}^{-\infty, \mathcal{E}}(X)+\Psi^{-\infty, E_{l b}, E_{r b}}(X) .
$$

Remark 1. This is not the same as the original definition of the full calculus as presented in [35, Def. 5.51]. Melrose always sets $E_{f f}=\mathbb{N}_{0}$. The definition we use is the one that is most suitable for this paper.

Given $a \in \mathbb{R}$ and an index set $E$, we write $E \geq a$ if $(z, k) \in E \Rightarrow \operatorname{Re} z \geq a$ and $(a, k) \notin E$ for any $k>0$. We write $E>a$ if $(z, k) \in E \Rightarrow \operatorname{Re} z>a$.

If $E_{l b}>\beta, E_{r b}>-\alpha$, and $\alpha+E_{f f} \geq \beta$, then (see [31, Th. 3.25]) any operator $A \in \widetilde{\Psi}_{b}^{m, \mathcal{E}}(X)$ defines a continuous linear map

$$
A: x^{\alpha} H_{b}^{s}(X) \longrightarrow x^{\beta} H_{b}^{s-m}(X), \quad \text { for any } s \in \mathbb{R} .
$$

\subsection{Cone differential operators.}

Throughout the rest of this paper (unless stated otherwise), $x$ will denote a fixed boundary defining function on $X$ which gives a decomposition $X \cong$ $[0,1)_{x} \times Y$ near $Y=\partial X$.

A cone differential operator of order $m \in \mathbb{N}_{0}$ is an operator of the form $A=x^{-m} P$, where $P \in \operatorname{Diff}_{b}^{m}(X)$. The cone operator $A$ is said to be elliptic if $P$ is an elliptic $b$-differential operator. The boundary spectrum of an elliptic cone operator $A, \operatorname{spec}_{c}(A) \subseteq \mathbb{C}$, is the set of points $\tau \in \mathbb{C}$ where the normal operator $\widehat{P}(\tau)$ (see (3.2)) fails to be invertible.

Theorem 3.1. If $A \in x^{-m} \operatorname{Diff}_{b}^{m}(X)$ is elliptic and $\alpha \notin-\operatorname{Im} \operatorname{spec}_{c}(A)$, then for any $s \in \mathbb{R}, A: x^{\alpha} H_{b}^{s}(X) \longrightarrow x^{\alpha-m} H_{b}^{s-m}(X)$ is Fredholm, and its generalized inverse, $G_{\alpha}$, is in the full calculus: $G_{\alpha} \in x^{m} \widetilde{\Psi}_{b}^{-m, \mathcal{E}(\alpha)}(X)$. The index family $\mathcal{E}(\alpha)$ is defined in (3.8) below. Here, a b-density $0<\nu \in C^{\infty}\left(X, \Omega_{b}\right)$ is fixed, and the generalized inverse is defined by the equations

$$
A G_{\alpha}=\mathrm{Id}-\Pi_{1}, \quad G_{\alpha} A=\mathrm{Id}-\Pi_{0},
$$


where $\Pi_{0}$ and $\Pi_{1}$ are the orthogonal projections (with respect to $\nu$ ) onto the null space and off the range in $x^{\alpha} H_{b}^{s}(X)$ and $x^{\alpha-m} H_{b}^{s-m}(X)$ respectively. Moreover, $\Pi_{0} \in \Psi^{-\infty, \hat{E}^{+}(\alpha)+m, \hat{E}^{-}(\alpha)-m}(X)$ and $\Pi_{1} \in \Psi^{-\infty, \hat{E}^{+}(\alpha), \hat{E}^{-}(\alpha)}(X)$, where the index sets $\hat{E}^{ \pm}(\alpha)$ are defined in (3.7) below.

Proof. (Outline) Let $B=A x^{m} \in \operatorname{Diff}_{b}^{m}(X)$. Note that ${ }^{b} \sigma\left(x^{m} A\right)={ }^{b} \sigma(B)$. Thus, $B$ is an elliptic $b$-differential operator. Also note that $\widehat{x^{m} A}(\tau)=$ $\widehat{B}(\tau+i m)$. It follows that $\widehat{B}(\tau)$ is invertible for all $\tau \in \mathbb{C}$ with $-\operatorname{Im} \tau=$ $\alpha-m$. Hence, by [31, Th. 4.4], $B: x^{\alpha-m} H_{b}^{s}(X) \longrightarrow x^{\alpha-m} H_{b}^{s-m}(X)$ is Fredholm having a generalized inverse $H_{\alpha} \in \widetilde{\Psi}_{b}^{-m, \mathcal{E}(\alpha)}(X)$. Using the fact that $B=A x^{m}$ finishes the proof.

The index family $\mathcal{E}(\alpha)$ is defined as follows. We first define

$$
\begin{aligned}
E^{ \pm}(\alpha):= & \left\{(z, k) \mid \tau=\mp i z \in \operatorname{spec}_{c}(A)+i m\right. \\
& 1 \leq k+1 \leq \operatorname{ord}(\tau-i m), \text { and } \operatorname{Re} z> \pm(\alpha-m)\}
\end{aligned}
$$

Here, $\operatorname{ord}(\tau)$ denotes the order of the pole of $\widehat{x^{m} A}(\tau)^{-1}$ at $\tau \in \operatorname{spec}_{c}(A)$. The extended union of two index sets $E$ and $F$ is the index set $E \bar{\cup} F:=$ $E \cup F \cup\{(z, k+\ell+1) \mid(z, k) \in E,(z, \ell) \in F\}$. Let $\widehat{E}^{ \pm}(\alpha):=\bar{\bigcup}_{r \in \mathbb{N}_{0}}\left(E^{ \pm}(\alpha)+r\right)$; that is, explicitly

$$
\begin{aligned}
(3.7) \widehat{E}^{ \pm}(\alpha)= & \left\{(z+r, k) \mid r \in \mathbb{N}_{0}, \tau=\mp i z \in \operatorname{spec}_{c}(A)+i m,\right. \\
& \left.1 \leq k+1 \leq \sum_{\ell=0}^{r} \operatorname{ord}(\tau-i m \mp i \ell), \text { and Re } z> \pm(\alpha-m)\right\} .
\end{aligned}
$$

We define

$$
\begin{gathered}
E(\alpha):=\mathbb{N} \bar{U}\left(\widehat{E}^{+}(\alpha)+\widehat{E}^{-}(\alpha)\right) ; \\
\mathcal{E}(\alpha):=\left(\check{E}^{+}(\alpha), \check{E}^{-}(\alpha), E(\alpha)\right), \quad \text { where } \check{E}^{ \pm}(\alpha)=\widehat{E}^{ \pm}(\alpha) \bar{\cup} \widehat{E}^{ \pm}(\alpha) .
\end{gathered}
$$

The next theorem describes the behavior of the resolvent for finite $\lambda$.

Theorem 3.2 (Analytic Fredholm Theory). Let $A \in x^{-m} \operatorname{Diff}_{b}^{m}(X)$ be elliptic and assume that $\alpha \notin-\operatorname{Im}_{\operatorname{spec}}(A)$. Then for any $s \in \mathbb{R}$,

$$
A-\lambda: x^{\alpha} H_{b}^{s}(X) \longrightarrow x^{\alpha-m} H_{b}^{s-m}(X)
$$

is either never invertible or else, $\mathbb{C} \ni \lambda \mapsto(A-\lambda)^{-1}$ is meromorphic with values in $x^{m} \widetilde{\Psi}_{b}^{-m, \mathcal{E}(\alpha)}(X)$ having only finite rank singularities. 
Proof. If $A$ is elliptic and $\alpha \notin-\operatorname{Im} \operatorname{spec}_{c}(A)$, then as ${ }^{b} \sigma\left(x^{m}(A-\lambda)\right)=$ ${ }^{b} \sigma\left(x^{m} A\right)$ and $\operatorname{spec}_{c}(A-\lambda)=\operatorname{spec}_{c}(A), A-\lambda$ is elliptic and $\alpha \notin-\operatorname{Im} \operatorname{spec}_{c}(A-$ $\lambda)$. Now following the proof of Theorem 3.1 and using the standard arguments for analytic Fredholm theory for pseudodifferential operators on closed manifolds completes the proof.

Example: The Cone Laplacian. A Riemannian metric $g$ on the interior of $X$ is called a conic metric if on the decomposition $X \cong[0,1)_{x} \times Y$ near $Y$, we can write $g=d x^{2}+x^{2} h(x)$, where $h(x)$ is a family of Riemannian metrics on $Y$ depending smoothly on $x$. Note that the Riemannian measure $d g$ is a smooth density in $x^{n} C^{\infty}\left(X, \Omega_{b}\right)$. A computation shows that on $[0,1)_{x} \times Y$, the Laplacian $\Delta_{c}$ associated to such a metric is of the form

$$
\Delta_{c}=x^{-2}\left[\left(x D_{x}\right)^{2}-i(n-2) x D_{x}+\Delta_{h}-\frac{x^{2}}{\sqrt{\operatorname{det} h(x)}}\left(\partial_{x} \sqrt{\operatorname{det} h(x)}\right) \cdot \partial_{x}\right]
$$

where $\Delta_{h}=\Delta_{h(x)}$ is the Laplacian on $Y$ associated to the metric $h(x)$. In particular, $\Delta_{c} \in x^{-2} \operatorname{Diff}_{b}^{2}(X)$. Note that as $d g \in x^{n} C^{\infty}\left(X, \Omega_{b}\right)$, we have $x^{-n / 2} L_{b}^{2}(X) \equiv L^{2}(X, d g)$, the square integrable functions with respect to $d g$. It follows that $\Delta_{c}: x^{-n / 2+2} H_{b}^{2}(X) \longrightarrow x^{-n / 2} L_{b}^{2}(X)$ is symmetric and non-negative (with respect to the $b$-measure $x^{-n} d g$ ).

Let $0 \leq \lambda_{1}<\lambda_{2}<\cdots$ be the eigenvalues of $\Delta_{h(0)} \in \operatorname{Diff}^{2}(Y)$. Then from (3.9), it follows that $\operatorname{spec}_{c}\left(\Delta_{c}\right)=i \cdot\left\{\mu_{j}\right\}_{j= \pm 1, \pm 2, \ldots}$, where

$$
\mu_{ \pm j}=\frac{n-2 \pm \sqrt{(n-2)^{2}+4 \lambda_{j}}}{2}, \quad \text { where } j \in \mathbb{N} .
$$

One can check that $i(0, n-2) \cap \operatorname{spec}_{c}\left(\Delta_{c}\right)=\varnothing$. Fix $n \geq 5$. Then, this gap in $\operatorname{spec}_{c}\left(\Delta_{c}\right)$ implies that $-n / 2+2 \notin-\operatorname{Im} \operatorname{spec}_{c}\left(\Delta_{c}\right)$. Moreover, using the generalized inverse of Theorem 3.1 and the gap in $\operatorname{spec}_{c}\left(\Delta_{c}\right)$, it is straightforward to show that $\Delta_{c}: x^{-n / 2+2} H_{b}^{2}(X) \longrightarrow x^{-n / 2} L_{b}^{2}(X)$ is self-adjoint. Hence, by Theorem 3.2 , for any $s \in \mathbb{R}$,

$$
\Delta_{c}-\lambda: x^{-n / 2+2} H_{b}^{s}(X) \longrightarrow x^{-n / 2} H_{b}^{s-m}(X)
$$

is invertible for $\lambda \notin \overline{\mathbb{R}^{+}}$, and $\mathbb{C} \ni \lambda \mapsto\left(\Delta_{c}-\lambda\right)^{-1}$ is meromorphic with values in $x^{2} \widetilde{\Psi}_{b}^{-2, \mathcal{E}(\alpha)}(X)$ with $\alpha=-n / 2+2$, having only finite rank singularities. Here, the index family $\mathcal{E}(\alpha)$ is defined in terms of $\operatorname{spec}_{c}\left(\Delta_{c}\right)$, see (3.8). Theorem 6.1 describes $\left(\Delta_{c}-\lambda\right)^{-1}$ as an element of a parameter dependent space of operators, and Theorem 8.2 describes how the singularities of the Schwartz kernel of $\left(\Delta_{c}-\lambda\right)^{-1}$ accumulate near the diagonal as $\lambda \rightarrow \infty$. 


\section{The small tempered calculus.}

\subsection{The small calculus.}

Before defining our basic space of parameter-dependent operators, see Definition 2 below, we review their corresponding symbols. These symbols are found throughout the literature, cf. [29] or Gilkey's book [17].

Let $\Lambda \subseteq \mathbb{C}$ be a closed sector (closed angle with vertex at 0 ) and let $d \in \mathbb{N}$. The space $S_{\Lambda}^{m, d}\left(\mathbb{R}^{n}\right)$, where $m \in \mathbb{R}$, consists of those functions $a(\lambda, \xi) \in C^{\infty}\left(\Lambda \times \mathbb{R}^{n}\right)$ satisfying the following estimates: for each $\alpha, \beta$, there exists a $C>0$ such that

$$
\left|\partial_{\lambda}^{\alpha} \partial_{\xi}^{\beta} a(\lambda, \xi)\right| \leq C\left(1+|\lambda|^{1 / d}+|\xi|\right)^{m-d|\alpha|-|\beta|} .
$$

The "classical" subspace $S_{\Lambda, c \ell}^{m, d}\left(\mathbb{R}^{n}\right)$ consists of symbols $a(\lambda, \xi) \in S_{\Lambda}^{m, d}\left(\mathbb{R}^{n}\right)$ such that

$$
a(\lambda, \xi) \sim \sum_{k=0}^{\infty} \chi(\lambda, \xi) a_{m-k}(\lambda, \xi),
$$

where $\chi(\lambda, \xi) \in C^{\infty}\left(\Lambda \times \mathbb{R}^{n}\right)$ with $\chi(\lambda, \xi) \equiv 0$ near $(\lambda, \xi)=0$ and $\chi(\lambda, \xi) \equiv 1$ outside a neighborhood of 0 , and where $a_{m-k}(\lambda, \xi) \in C^{\infty}\left(\Lambda \times \mathbb{R}^{n} \backslash(0,0)\right)$ is anisotropic homogeneous of degree $m-k$ :

$$
a_{m-k}\left(\delta^{d} \lambda, \delta \xi\right)=\delta^{m-k} a_{m-k}(\lambda, \xi), \quad \text { for all } \delta>0 .
$$

The asymptotic sum (4.2) means that for each $N \in \mathbb{N}$, we have $a(\lambda, \xi)-$ $\sum_{k=0}^{N-1} \chi(\lambda, \xi) a_{m-k}(\lambda, \xi) \in S_{\Lambda}^{m-N, d}\left(\mathbb{R}^{n}\right)$.

Of course, the "canonical" example of a classical symbol is the local symbol for the resolvent. The following lemma is straightforward to verify.

Lemma 4.1. Let $a(\xi)$ be a homogeneous polynomial of degree $m \in \mathbb{N}$ such that $a(\xi)$ never takes values in $\Lambda$ for $\xi \neq 0$, and let $\chi(\lambda, \xi)$ be a cut-off function as defined above. Then, $\chi(\lambda, \xi)(a(\xi)-\lambda)^{-1} \in S_{\Lambda, c \ell}^{-m, m}\left(\mathbb{R}^{n}\right)$.

Throughout this paper, $\rho$ will always denote a boundary defining function for $f f\left(X_{b}^{2}\right)$. Let $0<\nu \in C^{\infty}\left(X, \Omega_{b}\right)$. Recall that $\nu^{\prime}$ denotes the lift of $\nu$ to $X^{2}$ under the projection $X^{2} \ni\left(y, y^{\prime}\right) \mapsto y^{\prime} \in X$.

Definition 2. (cf. Definition 1) Let $m \in \mathbb{R}$. Then the small calculus of tempered cone pseudodifferential operators of degree $m, d$, denoted by $\Psi_{c, \Lambda}^{m, d}(X)$, consists of those parameter-dependent operators $A(\lambda)$ with a Schwartz kernel $K_{A(\lambda)}$ satisfying the following two conditions: 
(1) Given $\varphi \in C_{c}^{\infty}\left(X_{b}^{2} \backslash \Delta_{b}\right)$, the kernel $\varphi K_{A(\lambda)}$ is of the form $k\left(\rho^{d} \lambda, p\right) \nu^{\prime}$, where $k(\lambda, p) \in C^{\infty}\left(\Lambda \times X_{b}^{2}\right)$ and vanishes to infinite order at $\lambda=\infty$ (that is, with all derivatives as $|\lambda| \rightarrow \infty$ in $\Lambda$ ), and at $p \in l b$ and $p \in r b$.

(2) Given a coordinate patch of $X_{b}^{2}$ near $\Delta_{b}$ of the form $\mathcal{U}_{y} \times \mathbb{R}_{z}^{n}$ such that $\Delta_{b} \cong \mathcal{U} \times\{0\}($ cf. $(2.3))$, and given $\varphi \in C_{c}^{\infty}\left(\mathcal{U} \times \mathbb{R}^{n}\right)$, we have

$$
\varphi K_{A(\lambda)}=\int_{\mathbb{R}^{n}} e^{i z \cdot \xi} a\left(\rho^{d} \lambda, y, \xi\right) d \xi \cdot \nu^{\prime}
$$

where $y \mapsto a(\lambda, y, \xi) \in C^{\infty}\left(\mathcal{U} ; S_{\Lambda, c \ell}^{m, d}\left(\mathbb{R}^{n}\right)\right)$.

One can check that this space of operators is defined independent of the choice of $\rho$. We define $\Psi_{c, \Lambda}^{-\infty, d}(X):=\bigcap_{m \in \mathbb{R}} \Psi_{c, \Lambda}^{m, d}(X)$. Observe that Lemma 4.1 suggests that $(A-\lambda)^{-1} \in x^{m} \Psi_{c, \Lambda}^{-m, m}(X)$ if $A \in x^{-m} \operatorname{Diff}_{b}^{m}(X)$. This is almost true, except for some "smoothing" type operators introduced in Section 5.

\subsection{Properties of the small calculus.}

Let $\Lambda \subseteq \mathbb{C}$ be a closed sector and let $d \in \mathbb{N}$. Recall that $x$ represents a fixed boundary defining function on $X$.

Proposition 4.2. Let $m \in \mathbb{R}$. Then,

(A) for any $k \in \mathbb{N}, \Psi_{c, \Lambda}^{m, d}(X) \subseteq \Psi_{c, \Lambda}^{m+k, d}(X)$;

(B) for any $\alpha, \partial_{\lambda}^{\alpha} \Psi_{c, \Lambda}^{m, d}(X) \subseteq x^{|\alpha| d} \Psi_{c, \Lambda}^{m-d|\alpha|, d}(X)$;

(C) Let $f: \Lambda \times X \longrightarrow \Lambda \times X$ be the map $f(\lambda, p)=\left(x(p)^{d} \lambda, p\right)$. Then given an index set $F$, any operator $A \in \Psi_{c, \Lambda}^{m, d}(X)$ defines a continuous map

$$
A: \mathcal{A}_{p h g}^{F}(X) \longrightarrow f^{*} S_{c \ell}^{m / d}\left(\Lambda ; \mathcal{A}_{p h g}^{F}(X)\right)
$$

Here, $S_{c l}^{m / d}\left(\Lambda ; \mathcal{A}_{p h g}^{F}(X)\right)$ denotes the classical symbols of degree $m / d$ on $\Lambda$ with values in the Fréchet space $\mathcal{A}_{\text {phg }}^{F}(X)$.

Proof. Properties $(A)$ and $(B)$ follow directly from Definition 2. We now prove $(C)$. Let $A \in \Psi_{c, \Lambda}^{m, d}(X)$ and $u \in \mathcal{A}_{p h g}^{F}(X)$. Then following the discussion around (1.3) in the Appendix, to prove $(C)$, we must show that $\mu A u=$ 
$\left(\pi_{L, b}\right)_{*}\left(\pi_{L, b}^{*} \mu \pi_{R, b}^{*} u K_{A}\right) \in f^{*} S_{c l}^{m / d}\left(\Lambda ; \mathcal{A}_{p h g}^{F}\left(X, \Omega_{b}\right)\right)$, where $\mu \in C^{\infty}\left(X, \Omega_{b}\right)$. To prove this, we will use local coordinates, and for simplicity, we assume that $K_{A}$ is supported near $f f\left(X_{b}^{2}\right)$. In what follows, we will rely heavily on the properties of $K_{A}$ as described in Definition 2.

Let $\mathcal{V} \cong \mathbb{R}^{n-1}$ be a coordinate patch on $Y$. Then $X \cong[0,1)_{x} \times \mathcal{V}$ near $Y$, and $X_{b}^{2} \cong[0,1)_{b}^{2} \times \mathcal{V}^{2}$ near ff. Moreover, cf. Figure 1 in Section 2.2 and Equation (2.3), $\left(s, x^{\prime}, y, y^{\prime}\right)$, where $s=x / x^{\prime}$, are coordinates on $[0,1)_{b}^{2} \times \mathcal{V}^{2}$ near $l b ;\left(x, t, y, y^{\prime}\right)$, where $t=x^{\prime} / x$, are coordinates near $r b$; and $(x, y, z)$, where $z=\left(\log \left(x / x^{\prime}\right), y-y^{\prime}\right)$, are coordinates near $\Delta_{b}$, with $\Delta_{b}=\{z=0\}$.

Assume first that the Schwartz kernel $K_{A}$ is supported near $l b$. Then according to Definition 2, in the coordinates $\left(s, x^{\prime}, y, y^{\prime}\right)$ we can write

$$
\pi_{L, b}^{*} \mu \pi_{R, b}^{*} u K_{A}=B_{1}\left(\left(x^{\prime}\right)^{d} \lambda, s, x^{\prime}, y, y^{\prime}\right)\left|\frac{d s}{s} \frac{d x^{\prime}}{x^{\prime}} d y d y^{\prime}\right|,
$$

where $B_{1}\left(\lambda, s, x^{\prime}, y, y^{\prime}\right)$ vanishes to infinite order at $s=0, \lambda=\infty$, and has an expansion at $x^{\prime}=0$ with index set $F$. Observe that $\pi_{L, b}\left(s, x^{\prime}, y, y^{\prime}\right)=$ $\left(s x^{\prime}, y\right)$. Hence, by (1.1) of Lemma 1.1 in the Appendix, we have

$$
\left(\pi_{L, b}\right)_{*}\left(\pi_{L, b}^{*} \mu \pi_{R, b}^{*} u K_{A}\right)=\int B_{1}\left(\lambda / s^{d}, s, x / s, y, y^{\prime}\right) d y^{\prime} \frac{d s}{s} \cdot\left|\frac{d x}{x} d y\right| .
$$

Since $B_{1}\left(\lambda, s, x^{\prime}, y, y^{\prime}\right)$ vanishes identically at $\lambda=\infty$ and $s=0$, and at $x^{\prime}=0$ has an asymptotic expansion with index set $F$, it follows that $\left(\pi_{L, b}\right)_{*}\left(\pi_{L, b}^{*} \mu \pi_{R, b}^{*} u K_{A}\right) \in S^{-\infty}\left(\Lambda ; \mathcal{A}_{p h g}^{F}\left(X, \Omega_{b}\right)\right) \subseteq f^{*} S_{c \ell}^{m / d}\left(\Lambda ; \mathcal{A}_{p h g}^{F}\left(X, \Omega_{b}\right)\right)$.

Assume now that $K_{A}$ is supported near $r b$. Then in the coordinates $\left(x, t, y, y^{\prime}\right)$, we can write

$$
\pi_{L, b}^{*} \mu \pi_{R, b}^{*} u K_{A}=B_{2}\left(x^{d} \lambda, x, t, y, y^{\prime}\right)\left|\frac{d x}{x} \frac{d t}{t} d y d y^{\prime}\right|,
$$

where $B_{2}\left(\lambda, x, t, y, y^{\prime}\right)$ vanishes to infinite order at $\lambda=\infty$ and $t=0$, and has an expansion at $x=0$ with index set $F$. In these coordinates, $\pi_{L, b}\left(x, t, y, y^{\prime}\right)=(x, y)$ is a fibration. Hence,

$$
\left(\pi_{L, b}\right)_{*}\left(\pi_{L, b}^{*} \mu \pi_{R, b}^{*} u K_{A}\right)=\int B_{2}\left(x^{d} \lambda, x, t, y, y^{\prime}\right) d y^{\prime} \frac{d t}{t} \cdot\left|\frac{d x}{x} d y\right| .
$$

The asymptotic properties of $B_{2}$ imply that $\left(\pi_{L, b}\right)_{*}\left(\pi_{L, b}^{*} \mu \pi_{R, b}^{*} u K_{A}\right) \in$ $f^{*} S^{-\infty}\left(\Lambda ; \mathcal{A}_{p h g}^{F}\left(X, \Omega_{b}\right)\right) \subseteq f^{*} S_{c \ell}^{m / d}\left(\Lambda ; \mathcal{A}_{p h g}^{F}\left(X, \Omega_{b}\right)\right)$.

Now assume that $K_{A}$ is supported near $\Delta_{b}$. Then we can write

$$
\pi_{L, b}^{*} \mu \pi_{R, b}^{*} u K_{A}=\int e^{i z \cdot \xi} a\left(x^{d} \lambda, x, y, \xi\right) d \xi \cdot\left|\frac{d x}{x} d z d y\right|,
$$


where $a(\lambda, x, y, \xi)$ has an expansion at $x=0$ with index set $F$, and where $(\lambda, \xi) \mapsto a(\lambda, x, y, \xi) \in S_{\Lambda, c \ell}^{m, d}\left(\mathbb{R}_{\xi}^{n}\right)$. Since $\pi_{L, b}(x, y, z)=(x, y)$, it follows that

$$
\left(\pi_{L, b}\right)_{*}\left(\pi_{L, b}^{*} \mu \pi_{R, b}^{*} u K_{A}\right)=a\left(x^{d} \lambda, x, y, 0\right)\left|\frac{d x}{x} d y\right| .
$$

Thus, $\left(\pi_{L, b}\right)_{*}\left(\pi_{L, b}^{*} \mu \pi_{R, b}^{*} u K_{A}\right) \in f^{*} S^{m / d}\left(\Lambda ; \mathcal{A}_{p h g}^{F}\left(X, \Omega_{b}\right)\right)$ here as well.

\subsection{Composition.}

We begin by proving composition for the $-\infty$ operators.

Lemma 4.3. We have $\Psi_{c, \Lambda}^{-\infty, d}(X) \circ \Psi_{c, \Lambda}^{-\infty, d}(X) \subseteq \Psi_{c, \Lambda}^{-\infty, d}(X)$.

Proof. Let $A \in \Psi_{c, \Lambda}^{-\infty, d}(X), B \in \Psi_{c, \Lambda}^{-\infty, d}(X)$, and fix $0<\mu \in C^{\infty}\left(X, \Omega_{b}\right)$. To show that $A B \in \Psi_{c, \Lambda}^{-\infty, d}(X)$, we will use the formula

$$
\mu K_{A B}=\left(\pi_{C, b}\right)_{*}\left(\pi_{C, b}^{*} \mu \pi_{F, b}^{*} K_{A} \pi_{S, b}^{*} K_{B}\right) .
$$

See (1.6) in the Appendix for the definitions of $\pi_{C, b}, \pi_{F, b}$, and $\pi_{S, b}$; and see the derivation of Equation (1.7) in the Appendix for the proof of (4.3).

We will use local coordinates to analyze (4.3), and for simplicity, we assume that the Schwartz kernels of $A$ and $B$ are supported near $f f\left(X_{b}^{2}\right)$. Thus, if $\mathcal{V} \cong \mathbb{R}^{n-1}$ is a coordinate patch on $Y=\partial X$, we can decompose $X \cong$ $[0,1)_{x} \times \mathcal{V}_{y}$. Note that $X_{b}^{2} \cong[0,1)_{b}^{2} \times \mathcal{V}^{2}$ near ff $\left(X_{b}^{2}\right)$ and $X_{b}^{3} \cong[0,1)_{b}^{3} \times \mathcal{V}^{3}$ near $f f\left(X_{b}^{3}\right)$. (See the middle picture in Figure 2 for a picture of $X_{b}^{3}$.)

We will use the following coordinates on $[0,1)_{b}^{2} \times \mathcal{V}^{2}$ (see Figure 1):

$$
\left(s, x^{\prime}, y, y^{\prime}\right), \text { where } s=x / x^{\prime}, \text { are coordinates near } l b
$$

$$
\left(x, t, y, y^{\prime}\right) \text {, where } t=x^{\prime} / x, \text { are coordinates near } r b .
$$

Let $x, x^{\prime}, x^{\prime \prime}$ be the coordinates on the left, middle, and right factors of $[0,1)^{3}$ and $y, y^{\prime}, y^{\prime \prime}$ be the coordinates on the left, middle, and right factors of $\mathcal{V}^{3}$. Assume that $\mu=\left|\frac{d x}{x} d y\right|$.

Step 1: We analyze $\mu K_{A B}$ near the intersection of $m b$, ff, and $f s$ of $X_{b}^{3}$. Here, we may use the coordinates $\left(s, t, x^{\prime \prime}, y, y^{\prime}, y^{\prime \prime}\right)$, where $s=x / x^{\prime \prime}$ and $t=x^{\prime} / x$ (see the left-hand picture in Figure 3). Near the intersection of $m b$, 


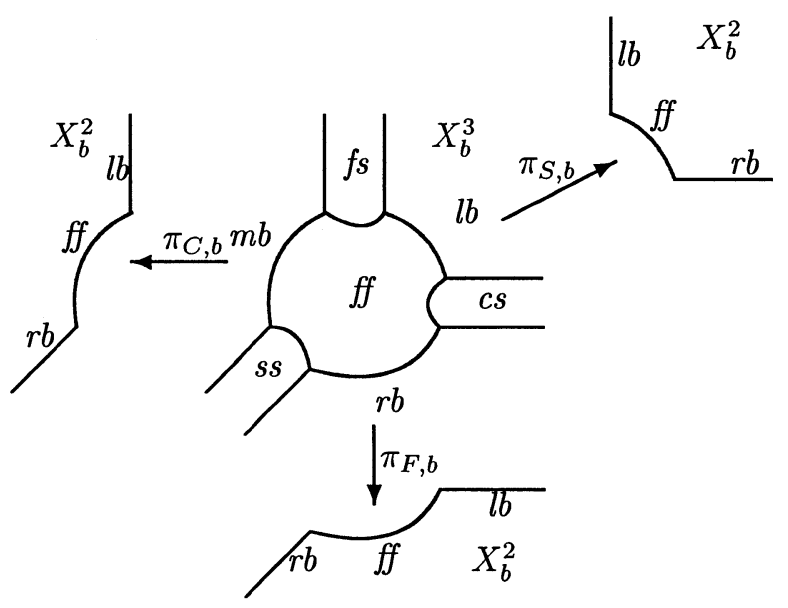

Figure 2: $X_{b}^{3}$ and the projections $\pi_{F, b}, \pi_{S, b}$, and $\pi_{C, b}$.
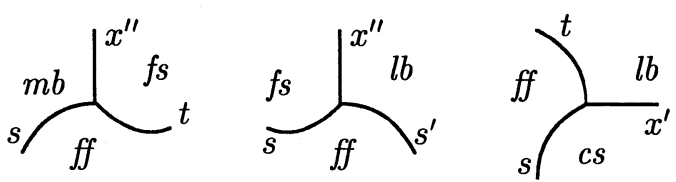

Figure 3: Various coordinate patches on $X_{b}^{3}$.

$f f$, and $f s, \pi_{C, b}$ and $\pi_{S, b}$ map near $l b$ in $X_{b}^{2}$ and $\pi_{F, b}$ maps near $r b$ in $X_{b}^{2}$. Moreover, in the coordinates (4.4) on $X_{b}^{2}$ near $l b$, we have

$$
\pi_{S, b}\left(s, t, x^{\prime \prime}, y, y^{\prime}, y^{\prime \prime}\right)=\left(s t, x^{\prime \prime}, y^{\prime}, y^{\prime \prime}\right) ; \pi_{C, b}\left(s, t, x^{\prime \prime}, y, y^{\prime}, y^{\prime \prime}\right)=\left(s, x^{\prime \prime}, y, y^{\prime \prime}\right)
$$

and in the coordinates $(4.5)$ on $X_{b}^{2}$ near $r b$, we have

$$
\pi_{F, b}\left(s, t, x^{\prime \prime}, y, y^{\prime}, y^{\prime \prime}\right)=\left(s x^{\prime \prime}, t, y, y^{\prime}\right)
$$

Near $r b$ in $X_{b}^{2}, K_{A}=A\left(x^{d} \lambda, x, t, y, y^{\prime}\right)\left|\frac{d x^{\prime}}{x^{\prime}} d y^{\prime}\right|$, where $A\left(\lambda, x, t, y, y^{\prime}\right)$ is smooth in all variables, and vanishes identically at $\lambda=\infty$ and $t=0$. Near $l b$ in $X_{b}^{2}, K_{B}=B\left(\left(x^{\prime}\right)^{d} \lambda, s, x^{\prime}, y, y^{\prime}\right)\left|\frac{d x^{\prime}}{x^{\prime}} d y^{\prime}\right|$, where $B\left(\lambda, s, x^{\prime}, y, y^{\prime}\right)$ is smooth in all variables, and vanishes identically at $\lambda=\infty$ and $s=0$. Using the formulas for $\pi_{S, b}$ and $\pi_{F, b}$ in (4.6) and (4.7), it follows that near the intersection of $m b, f f$, and $f s$ of $X_{b}^{3}$,

$$
\begin{aligned}
& \pi_{C, b}^{*} \mu \pi_{F, b}^{*} K_{A} \pi_{S, b}^{*} K_{B}= \\
& \quad A\left(\left(s x^{\prime \prime}\right)^{d} \lambda, s x^{\prime \prime}, t, y, y^{\prime}\right) B\left(\left(x^{\prime \prime}\right)^{d} \lambda, s t, x^{\prime \prime}, y^{\prime}, y^{\prime \prime}\right)\left|\frac{d s d t d x^{\prime \prime}}{s t x^{\prime \prime}} d y d y^{\prime} d y^{\prime \prime}\right|
\end{aligned}
$$


Hence, as $\pi_{C, b}\left(s, t, x^{\prime \prime}, y, y^{\prime}, y^{\prime \prime}\right)=\left(s, x^{\prime \prime}, y, y^{\prime \prime}\right)$ is a fibration,

$$
\begin{aligned}
& \mu K_{A B}=\left(\pi_{C, b}\right)_{*}\left(\pi_{C, b}^{*} \mu \pi_{F, b}^{*} K_{A} \pi_{S, b}^{*} K_{B}\right) \\
& \quad=\int A\left(\left(s x^{\prime}\right)^{d} \lambda, s x^{\prime}, t, y, y^{\prime \prime}\right) B\left(\left(x^{\prime}\right)^{d} \lambda, s t, x^{\prime}, y^{\prime \prime}, y^{\prime}\right) \frac{d t}{t} d y^{\prime \prime} \cdot\left|\frac{d s d x^{\prime}}{s x^{\prime}} d y d y^{\prime}\right| \\
& \quad=C\left(\left(x^{\prime}\right)^{d} \lambda, s, x^{\prime}, y, y^{\prime}\right)\left|\frac{d s d x^{\prime}}{s x^{\prime}} d y d y^{\prime}\right|
\end{aligned}
$$

where $C\left(\lambda, s, x^{\prime}, y, y^{\prime}\right)=\int A\left(s^{d} \lambda, s x^{\prime}, t, y, y^{\prime \prime}\right) B\left(\lambda, s t, x^{\prime}, y^{\prime \prime}, y^{\prime}\right) \frac{d t}{t} d y^{\prime \prime}$. Now, since $A\left(\lambda, x, t, y, y^{\prime}\right)$ is smooth in all variables, vanishing identically at $\lambda=\infty$ and $t=0$, and since $B\left(\lambda, s, x^{\prime}, y, y^{\prime}\right)$ is smooth in all variables, vanishing identically at $\lambda=\infty$ and $s=0$, it follows that $C\left(\lambda, s, x^{\prime}, y, y^{\prime}\right)$ is smooth in all variables, vanishing identically at $\lambda=\infty$ and $s=0$.

Step 2: We analyze $\mu K_{A B}$ near the intersection of $f s, f f$, and $l b$ of $X_{b}^{3}$. Here, we may use the coordinates $\left(s, s^{\prime}, x^{\prime \prime}, y, y^{\prime}, y^{\prime \prime}\right)$, where $s=x / x^{\prime}$ and $s^{\prime}=x^{\prime} / x^{\prime \prime}$ (see the middle picture in Figure 3 ). Near the intersection of $f s, f f$, and $l b, \pi_{F, b}, \pi_{S, b}$, and $\pi_{C, b}$ all map near $l b$ in $X_{b}^{2}$. Moreover, in the coordinates (4.4) on $X_{b}^{2}$ near $l b$, we have

$$
\begin{aligned}
\pi_{F, b}\left(s, s^{\prime}, x^{\prime \prime}, y, y^{\prime}, y^{\prime \prime}\right) & =\left(s, s^{\prime} x^{\prime \prime}, y, y^{\prime}\right) \\
\pi_{S, b}\left(s, s^{\prime}, x^{\prime \prime}, y, y^{\prime}, y^{\prime \prime}\right) & =\left(s^{\prime}, x^{\prime \prime}, y^{\prime}, y^{\prime \prime}\right) \\
\pi_{C, b}\left(s, s^{\prime}, x^{\prime \prime}, y, y^{\prime}, y^{\prime \prime}\right) & =\left(s s^{\prime}, x^{\prime \prime}, y, y^{\prime \prime}\right)
\end{aligned}
$$

Near $l b$ in $X_{b}^{2}$, we can write $K_{A}=A\left(\left(x^{\prime}\right)^{d} \lambda, s, x^{\prime}, y, y^{\prime}\right)\left|\frac{d x^{\prime}}{x^{\prime}} d y^{\prime}\right|$, and $K_{B}=$ $B\left(\left(x^{\prime}\right)^{d} \lambda, s, x^{\prime}, y, y^{\prime}\right)\left|\frac{d x^{\prime}}{x^{\prime}} d y^{\prime}\right|$, where $A\left(\lambda, s, x^{\prime}, y, y^{\prime}\right)$ and $B\left(\lambda, s, x^{\prime}, y, y^{\prime}\right)$ are both smooth in all variables, and vanish identically at $\lambda=\infty$ and $s=0$. Using the formulas in (4.8), it follows that near the intersection of $f s, f f$, and $l b$ of $X_{b}^{3}$,

$$
\begin{aligned}
& \pi_{C, b}^{*} \mu \pi_{F, b}^{*} K_{A} \pi_{S, b}^{*} K_{B}= \\
& \quad A\left(\left(s^{\prime} x^{\prime \prime}\right)^{d} \lambda, s, s^{\prime} x^{\prime \prime}, y, y^{\prime}\right) B\left(\left(x^{\prime \prime}\right)^{d} \lambda, s^{\prime}, x^{\prime \prime}, y^{\prime}, y^{\prime \prime}\right)\left|\frac{d s d s^{\prime} d x^{\prime \prime}}{s s^{\prime} x^{\prime \prime}} d y d y^{\prime} d y^{\prime \prime}\right|
\end{aligned}
$$

Hence, as $\pi_{C, b}\left(s, s^{\prime}, x^{\prime \prime}, y, y^{\prime}, y^{\prime \prime}\right)=\left(s s^{\prime}, x^{\prime \prime}, y, y^{\prime \prime}\right)$, by (1.1) in the Appendix,

$$
\begin{aligned}
& \mu K_{A B}=\left(\pi_{C, b}\right)_{*}\left(\pi_{C, b}^{*} \mu \pi_{F, b}^{*} K_{A} \pi_{S, b}^{*} K_{B}\right) \\
& =\int A\left(\left(s^{\prime} x^{\prime}\right)^{d} \lambda, s / s^{\prime}, s^{\prime} x^{\prime}, y, y^{\prime \prime}\right) B\left(\left(x^{\prime}\right)^{d} \lambda, s^{\prime}, x^{\prime}, y^{\prime \prime}, y^{\prime}\right) \frac{d s^{\prime}}{s^{\prime}} d y^{\prime \prime} \cdot\left|\frac{d s d x^{\prime}}{s x^{\prime}} d y d y^{\prime}\right| \\
& =C\left(\left(x^{\prime}\right)^{d} \lambda, s, x^{\prime}, y, y^{\prime}\right)\left|\frac{d s d x^{\prime}}{s x^{\prime}} d y d y^{\prime}\right|
\end{aligned}
$$


where $C\left(\lambda, s, x^{\prime}, y, y^{\prime}\right)=\int c\left(\lambda, s / s^{\prime}, s^{\prime}, x^{\prime}, y, y^{\prime}\right) \frac{d s^{\prime}}{s^{\prime}}$, with

$$
c\left(\lambda, s, s^{\prime}, x^{\prime}, y, y^{\prime}\right)=\int A\left(\left(s^{\prime}\right)^{d} \lambda, s, s^{\prime} x^{\prime}, y, y^{\prime \prime}\right) B\left(\lambda, s^{\prime}, x^{\prime}, y^{\prime \prime}, y^{\prime}\right) d y^{\prime \prime} .
$$

Now, as $A\left(\lambda, s, x^{\prime}, y, y^{\prime}\right)$ and $B\left(\lambda, s, x^{\prime}, y, y^{\prime}\right)$ are smooth in all variables, vanishing identically at $\lambda=\infty$ and $s=0$, by Lemma 1.1 in the Appendix, it follows that $C\left(\lambda, s, x^{\prime}, y, y^{\prime}\right)$ is smooth in all variables, vanishing identically at $\lambda=\infty$ and $s=0$.

Step 3: We analyze $\mu K_{A B}$ near the intersection of $f f, c s$, and $l b$ of $X_{b}^{3}$. Here, we may use the coordinates $\left(s, x^{\prime}, t, y, y^{\prime}, y^{\prime \prime}\right)$, where $s=x / x^{\prime \prime}$ and $t=x^{\prime \prime} / x^{\prime}$ (see the right-hand picture in Figure 3). Near the intersection of $f f, c s$, and $l b, \pi_{C, b}$ and $\pi_{F, b}$ map near $l b$ in $X_{b}^{2}$ and $\pi_{S, b}$ maps near $r b$ in $X_{b}^{2}$. Moreover, in the coordinates (4.4) on $X_{b}^{2}$ near $l b$, we have

$$
\pi_{F, b}\left(s, x^{\prime}, t, y, y^{\prime}, y^{\prime \prime}\right)=\left(s t, x^{\prime}, y, y^{\prime}\right) ; \pi_{C, b}\left(s, x^{\prime}, t, y, y^{\prime}, y^{\prime \prime}\right)=\left(s, x^{\prime} t, y, y^{\prime \prime}\right),
$$

and in the coordinates (4.5) on $X_{b}^{2}$ near $r b$, we have

$$
\pi_{S, b}\left(s, x^{\prime}, t, y, y^{\prime}, y^{\prime \prime}\right)=\left(x^{\prime}, t, y, y^{\prime}\right) .
$$

Near $l b$ in $X_{b}^{2}, K_{A}=A\left(\left(x^{\prime}\right)^{d} \lambda, s, x^{\prime}, y, y^{\prime}\right)\left|\frac{d x^{\prime}}{x^{\prime}} d y^{\prime}\right|$, where $A\left(\lambda, s, x^{\prime}, y, y^{\prime}\right)$ is smooth in all variables, and vanishes identically at $\lambda=\infty$ and $s=0$. Near $r b$ in $X_{b}^{2}, K_{B}=B\left(x^{d} \lambda, x, t, y, y^{\prime}\right)\left|\frac{d x^{\prime}}{x^{\prime}} d y^{\prime}\right|$, where $B\left(\lambda, x, t, y, y^{\prime}\right)$ is smooth in all variables, and vanishes identically at $\lambda=\infty$ and $t=0$. Using the formulas for $\pi_{F, b}$ and $\pi_{S, b}$ in (4.9) and (4.10), it follows that near the intersection of $f f, c s$, and $l b$ of $X_{b}^{3}$,

$$
\begin{aligned}
& \pi_{C, b}^{*} \mu \pi_{F, b}^{*} K_{A} \pi_{S, b}^{*} K_{B}= \\
& \quad A\left(\left(x^{\prime}\right)^{d} \lambda, s t, x^{\prime}, y, y^{\prime}\right) B\left(\left(x^{\prime}\right)^{d} \lambda, x^{\prime}, t, y^{\prime}, y^{\prime \prime}\right)\left|\frac{d s d t d x^{\prime}}{s t x^{\prime}} d y d y^{\prime} d y^{\prime \prime}\right| .
\end{aligned}
$$

Hence, as $\pi_{C, b}\left(s, x^{\prime}, t, y, y^{\prime}, y^{\prime \prime}\right)=\left(s, x^{\prime} t, y, y^{\prime \prime}\right)$, by (1.1) in the Appendix,

$$
\begin{aligned}
& \mu K_{A B}=\left(\pi_{C, b}\right)_{*}\left(\pi_{C, b}^{*} \mu \pi_{F, b}^{*} K_{A} \pi_{S, b}^{*} K_{B}\right) \\
& =\int A\left(\left(x^{\prime} / t\right)^{d} \lambda, s t, x^{\prime} / t, y, y^{\prime \prime}\right) B\left(\left(x^{\prime} / t\right)^{d} \lambda, x^{\prime} / t, t, y^{\prime \prime}, y^{\prime}\right) \frac{d t}{t} d y^{\prime \prime} \cdot\left|\frac{d s d x^{\prime}}{s x^{\prime}} d y d y^{\prime}\right| \\
& =C\left(\left(x^{\prime}\right)^{d} \lambda, s, x^{\prime}, y, y^{\prime}\right)\left|\frac{d s d x^{\prime}}{s x^{\prime}} d y d y^{\prime}\right|,
\end{aligned}
$$

where $C\left(\lambda, s, x^{\prime}, y, y^{\prime}\right)=\int A\left(\lambda / t^{d}, s t, x^{\prime}, y, y^{\prime \prime}\right) B\left(\lambda / t^{d}, x^{\prime} / t, t, y^{\prime \prime}, y^{\prime}\right) \frac{d t}{t} d y^{\prime \prime}$. Now since $A\left(\lambda, s, x^{\prime}, y, y^{\prime}\right)$ is smooth in all variables, vanishing identically 
at $\lambda=\infty$ and $s=0$, and since $B\left(\lambda, x, t, y, y^{\prime}\right)$ is smooth in all variables, vanishing identically at $\lambda=\infty$ and $t=0$, it follows that $C\left(\lambda, s, x^{\prime}, y, y^{\prime}\right)$ is smooth in all variables, vanishing identically at $\lambda=\infty$ and $s=0$.

In summary, away from the faces $s s$ and $r b, \pi_{C, b}^{*} \mu \pi_{F, b}^{*} K_{A} \pi_{S, b}^{*} K_{B}$ pushes forward under $\pi_{C, b}$ to define the kernel of an element in $\mu \cdot \Psi_{c, \Lambda}^{-\infty, d, \mathcal{E}}(X)$. Similar arguments show the same thing away from $f s$ and $l b$. It follows that $K_{A B}$ defines the kernel of an element in $\Psi_{c, \Lambda}^{-\infty, d}(X)$, and hence, $A B \in$ $\Psi_{c, \Lambda}^{-\infty, d}(X)$.

Theorem 4.4. If $m, m^{\prime}, \alpha, \alpha^{\prime} \in \mathbb{R}$, then

$$
x^{\alpha} \Psi_{c, \Lambda}^{m, d}(X) \circ x^{\alpha^{\prime}} \Psi_{c, \Lambda}^{m^{\prime}, d}(X) \subseteq x^{\alpha+\alpha^{\prime}} \Psi_{c, \Lambda}^{m+m^{\prime}, d}(X) .
$$

Proof. Let $A \in \Psi_{c, \Lambda}^{m, d}(X), B \in \Psi_{c, \Lambda}^{m^{\prime}, d}(X)$. We will first show that $A B \in$ $\Psi_{c, \Lambda}^{m+m^{\prime}, d}(X)$. To do so, we first write $A=A_{1}+A_{2}$ and $B=B_{1}+B_{2}$, where $A_{1}, B_{1}$ are both supported away from $l b$ and $r b$, and where $A_{2}, B_{2}$ are both supported away from $\Delta_{b}$ and thus are elements of $\Psi_{c, \Lambda}^{-\infty, d}(X)$. Then,

$$
A B=A_{1} B_{1}+A_{2} B_{1}+A_{1} B_{2}+A_{2} B_{2} .
$$

By Lemma 4.3, $A_{2} B_{2} \in \Psi_{c, \Lambda}^{-\infty, d}(X)$. Let $0<\mu \in C^{\infty}\left(X, \Omega_{b}\right)$. Then as in Lemma 4.3 , the first three terms can be analyzed using local coordinates and the formula

$$
\mu K_{A B}=\left(\pi_{C, b}\right)_{*}\left(\pi_{C, b}^{*} \mu \pi_{F, b}^{*} K_{A} \pi_{S, b}^{*} K_{B}\right) .
$$

Let $X \cong \mathcal{U}=[0,1)_{x} \times \mathcal{V}$, where $\mathcal{V} \cong \mathbb{R}^{n-1}$ is a coordinate patch on $Y=\partial X$. Then, $X_{b}^{2} \cong[0,1)_{b}^{2} \times \mathcal{V}^{2}$ near $f f\left(X_{b}^{2}\right)$ and $X_{b}^{3} \cong[0,1)_{b}^{3} \times \mathcal{V}^{3}$ near $f f\left(X_{b}^{3}\right)$. Let $x, x^{\prime}$ be the coordinates on left and right factors of $[0,1)^{2}$ and $y, y^{\prime}$ be the coordinates on the left and right factors of $\mathcal{V}^{2}$. Similarly, let $x, x^{\prime}, x^{\prime \prime}$ be the coordinates on the left, middle, and right factors of $[0,1)^{3}$ and $y, y^{\prime}, y^{\prime \prime}$ be the coordinates on the left, middle, and right factors of $\mathcal{V}^{3}$.

Step 1: First consider $A_{1} B_{1}$. Near $\Delta_{b}$, we have (see (2.3)),

$$
\begin{aligned}
X_{b}^{2} \cong \mathcal{U} \times \mathbb{R}_{z}^{n}, z=\left(z_{1}, z^{\prime}\right), \text { where } z_{1}=\log \left(x^{\prime} / x\right), z^{\prime}=y^{\prime}-y, \\
\text { with } \Delta_{b} \cong \mathcal{U} \times\{z=0\} .
\end{aligned}
$$

Thus, in the coordinates (4.12), we can write

$$
K_{A_{1}}=\varphi(z) \int e^{i z \cdot \xi} a\left(x^{d} \lambda, x, y, \xi\right) d \xi \cdot \mu^{\prime} ; K_{B_{1}}=\int e^{i z \cdot \xi} b\left(x^{d} \lambda, x, y, \xi\right) d \xi \cdot \mu^{\prime}
$$




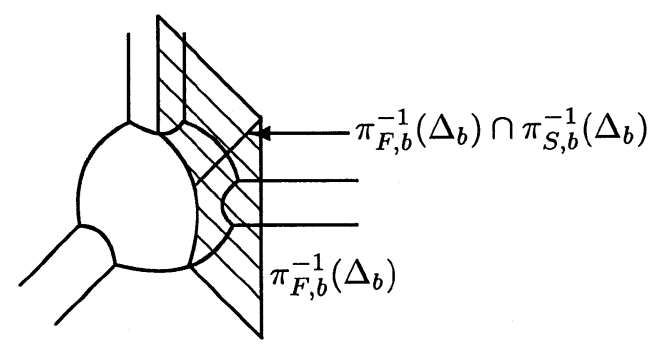

Figure 4: The manifold $X_{b}^{3}$ and the submanifolds $\pi_{F, b}^{-1}\left(\Delta_{b}\right) \cap \pi_{S, b}^{-1}\left(\Delta_{b}\right)$ and $\pi_{F, b}^{-1}\left(\Delta_{b}\right)$.

where $a(\lambda) \in C^{\infty}\left(\mathcal{U} ; S_{\Lambda, c \ell}^{m, d}\left(\mathbb{R}^{n}\right)\right), b(\lambda) \in C^{\infty}\left(\mathcal{U} ; S_{\Lambda, c l}^{m^{\prime}, d}\left(\mathbb{R}^{n}\right)\right), \varphi(z) \in C_{c}^{\infty}\left(\mathbb{R}^{n}\right)$, and where we may assume that $\mu=\left|\frac{d x}{x} d y\right|$. Near $\pi_{F, b}^{-1}\left(\Delta_{b}\right) \cap \pi_{S, b}^{-1}\left(\Delta_{b}\right)$ in $X_{b}^{3}$ (see Figure 4), we can use the coordinates $(x, y, z, w)$, where $z=$ $\left(\log \left(x^{\prime} / x\right), y^{\prime}-y\right)$ and $w=\left(\log \left(x^{\prime \prime} / x\right), y^{\prime \prime}-y\right)$. In these coordinates on $X_{b}^{3}$ and the coordinates (4.12) on $X_{b}^{2}$, we have

$$
\begin{gathered}
\pi_{C, b}(x, y, z, w)=(x, y, w) ; \pi_{F, b}(x, y, z, w)=(x, y, z) ; \\
\pi_{S, b}(x, y, z, w)=\left(x e^{z_{1}}, y+z^{\prime}, w-z\right) .
\end{gathered}
$$

Using (4.13) and (4.14), a short computation shows that

$$
\mu K_{A_{1} B_{1}}=\left(\pi_{C, b}\right)_{*}\left(\pi_{C, b}^{*} \mu \pi_{F, b}^{*} K_{A_{1}} \pi_{S, b}^{*} K_{B_{1}}\right)=\int e^{i z \cdot \xi} c\left(x^{d} \lambda, x, y, \xi\right) d \xi \cdot \mu \mu^{\prime},
$$

where $c(\lambda, x, y, \xi)=\int a(\lambda, x, y, \xi-\eta) \widetilde{b}(\lambda, x, y, \xi, \eta) d \eta$, with $\widetilde{b}(\lambda, x, y, \xi, \eta)=$ $\int e^{-i z \cdot \eta} \varphi(z) b\left(e^{d \cdot z_{1}} \lambda, x e^{z_{1}}, y+z^{\prime}, \xi\right) d z$ where $z=\left(z_{1}, z^{\prime}\right)$. Another computation shows that $c(\lambda, x, y, \xi) \in C^{\infty}\left(\mathcal{U} ; S_{\Lambda, c \ell}^{m+m^{\prime}, d}\left(\mathbb{R}^{n}\right)\right)$. Thus, $A_{1} B_{1} \in$ $\Psi_{c, \Lambda}^{m+m^{\prime}, d}(X)$.

Step 2: Now we work on the middle two terms of (4.11). Consider $A_{1} B_{2}$. Observe that $\pi_{F, b}^{-1}\left(\Delta_{b}\right) \cong X_{b}^{2} \times\{0\}_{u}$ in a decomposition (cf. Figure 4)

$$
X_{b}^{3} \cong X_{b}^{2} \times \mathbb{R}_{u}^{n}, \quad \text { where } \pi_{C, b}(p, u)=p, p \in X_{b}^{2} .
$$

Let $\rho$ be a boundary defining function for $f f\left(X_{b}^{2}\right)$. Then in the decomposition (4.15) near $\pi_{F, b}^{-1}\left(\Delta_{b}\right)$, we have (see Figure 4), $\pi_{F, b}^{*} \rho=\rho \rho_{l b}$ and $\pi_{S, b}^{*} \rho=\rho$, where $\rho_{l b}$ is a boundary defining function for $l b$ in $X_{b}^{2}$. Hence, as $A_{1} \in$ $\Psi_{c, \Lambda}^{m, d}(X)$ and $B_{2} \in \Psi_{c, \Lambda}^{-\infty, d}(X)$, it follows that in the decomposition (4.15), we can write

$$
\pi_{C, b}^{*} \mu \pi_{F, b}^{*} K_{A_{1}} \pi_{S, b}^{*} K_{B_{2}}=\int e^{i u \cdot \xi} c\left(\rho^{d} \rho_{l b}^{d} \lambda, \rho^{d} \lambda, p, \xi\right) d \xi \cdot|d u| \mu \mu^{\prime},
$$


where $X_{b}^{2} \ni p \mapsto c\left(\lambda, \lambda^{\prime}, p, \xi\right) \in S_{\Lambda_{\lambda}, c \ell}^{m, d}\left(\mathbb{R}_{\xi}^{n}\right) \widehat{\otimes} S^{-\infty}\left(\Lambda_{\lambda^{\prime}}\right)$ is smooth and vanishes to infinite order at $p \in l b$ and $p \in r b$ of $X_{b}^{2}$. Thus, as $\pi_{C, b}(p, u)=p$,

$$
\mu K_{A_{1} B_{2}}=\left(\pi_{C, b}\right)_{*}\left(\pi_{C, b}^{*} \mu \pi_{F, b}^{*} K_{A_{1}} \pi_{S, b}^{*} K_{B_{2}}\right)=c\left(\rho^{d} \rho_{l b}^{d} \lambda, \rho^{d} \lambda, p, 0\right) \cdot \mu \mu^{\prime} .
$$

The properties of $c\left(\lambda, \lambda^{\prime}, p, \xi\right)$ imply that $A_{1} B_{2} \in \Psi_{c, \Lambda}^{-\infty, d}(X)$. A similar argument shows that $A_{2} B_{1} \in \Psi_{c, \Lambda}^{-\infty, d}(X)$. Thus, $A B \in \Psi_{c, \Lambda}^{m+m^{\prime}, d}(X)$.

Now let $\alpha, \alpha^{\prime} \in \mathbb{R}$. Then,

$$
x^{\alpha} A \circ x^{\alpha^{\prime}} B=x^{\alpha+\alpha^{\prime}}\left(x^{-\alpha^{\prime}} A x^{\alpha^{\prime}}\right) \circ B=x^{\alpha+\alpha^{\prime}} A^{\prime} \circ B,
$$

where $A^{\prime}=\left(x^{-\alpha^{\prime}} A^{\prime} x^{\alpha^{\prime}}\right)$. Observe that $A^{\prime} \in \Psi_{c, \Lambda}^{m, d}(X)$. Thus, $x^{\alpha} A \circ x^{\alpha^{\prime}} B \in$ $x^{\alpha+\alpha^{\prime}} \Psi_{c, \Lambda}^{m+m^{\prime}, d}(X)$.

The following lemma is proved the same as the usual "asymptotic summation lemma" for pseudodifferential operators on a closed manifold.

Lemma 4.5. Given a sequence $A_{k} \in \Psi_{c, \Lambda}^{m-k, d}(X), k=0,1,2, \ldots$, there exists an $A \in \Psi_{c, \Lambda}^{m, d}(X)$ such that for all $N \in \mathbb{N}, A-\sum_{k=0}^{N-1} A_{k} \in \Psi_{c, \Lambda}^{m-N, d}(X)$; in which case, we write $A \sim \sum_{k=0}^{\infty} A_{k}$.

\section{The full calculus.}

\subsection{The full tempered calculus.}

In order to complete the definition of our parameter-dependent cone operators, we first need to define a blown-up manifold.

Let $d \in \mathbb{N}$. Henceforth, we will assume that $\Lambda \neq \mathbb{C}$. Thus, we can fix a branch of $\lambda^{1 / d}$ for $\lambda \in \Lambda$. We define $\Lambda_{d}:=\left\{\lambda^{1 / d} \mid \lambda \in \Lambda\right\}$. Then $\Lambda_{d}$ is also a closed sector in $\mathbb{C}$ and if $\zeta \in \Lambda_{d}$, then $\zeta^{d} \in \Lambda$.

We will denote by $\bar{\Lambda}_{d}$, the manifold $\Lambda_{d}$ radially compactified. We denote by $\partial_{\infty} \bar{\Lambda}_{d}$, the boundary "at $\lambda^{1 / d}=\infty$ ". Some convenient coordinates near $\partial_{\infty} \bar{\Lambda}_{d}$ are given by $r:=|\mu|$ and $\omega:=\mu /|\mu|$, where $\mu=1 / \lambda^{1 / d}$ for $\lambda \in \Lambda$.

We define

$$
\mathcal{T}_{d}:=\left[\bar{\Lambda}_{d} \times X_{b}^{2} ; \partial_{\infty} \bar{\Lambda}_{d} \times f f\right],
$$

see Figure 5. If $\beta: \mathcal{T}_{d} \longrightarrow \bar{\Lambda}_{d} \times X_{b}^{2}$ is the blow-down map, then we set $l b:=$ $\beta^{*}\left(\bar{\Lambda}_{d} \times l b\left(X_{b}^{2}\right)\right)$, left boundary; $r b:=\beta^{*}\left(\bar{\Lambda}_{d} \times r b\left(X_{b}^{2}\right)\right)$, right boundary; $f f:=\beta^{*}\left(\bar{\Lambda}_{d} \times f f\left(X_{b}^{2}\right)\right)$, front face; $f:=\beta^{*}\left(\partial_{\infty} \bar{\Lambda}_{d} \times f f\left(X_{b}^{2}\right)\right)$, face at infinity; and $b i:=\beta^{*}\left(\partial_{\infty} \bar{\Lambda}_{d} \times X_{b}^{2}\right)$, boundary at infinity. 


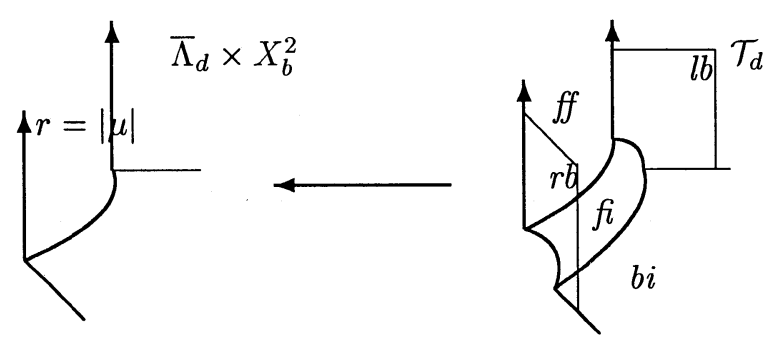

Figure 5: The manifold $\mathcal{T}_{d}$. Here, $\mu=1 / \lambda^{1 / d}$ for $\lambda \in \Lambda$ where $r=|\mu|=0$ defines $\partial_{\infty} \bar{\Lambda}_{d}$.

Let $0<\nu \in C^{\infty}\left(X, \Omega_{b}\right)$. Recall that $\nu^{\prime}$ denotes the lift of $\nu$ to $X^{2}$ under the projection $X^{2} \ni\left(y, y^{\prime}\right) \mapsto y^{\prime} \in X$.

Definition 3. Let $\mathcal{E}=\left(E_{l b}, E_{r b}, E_{f f}, E_{f i}\right)$ be an index family for $\mathcal{T}_{d}$ associated to the faces $(l b, r b, f f, f i)$. Then we define $\Psi_{c, \Lambda}^{-\infty, d, \mathcal{E}}(X)$ as those operators $A(\lambda)$ depending smoothly on $\lambda \in \Lambda$, that have a Schwartz kernel $K_{A(\lambda)}$ of the form $k \cdot \nu^{\prime}$, where $k \in \mathcal{A}_{\text {phg }}^{(\mathcal{E}, \varnothing)}\left(\mathcal{T}_{d}\right)$ with $\varnothing$ associated to bi. Thus, $k$ depends smoothly on $\lambda \in \Lambda$, and defines a function on $\mathcal{T}_{d}$ such that

1. At the hypersurface $b i, k$ vanishes to infinite order;

2. At any remaining hypersurface $H=l b, r b, f f$, or $f i, k$ has an expansion with index set $E_{H}$.

By definition, we have $A \in \Psi_{c, \Lambda}^{-\infty, d, \mathcal{E}}(X) \Rightarrow A \in C^{\infty}\left(\Lambda, \Psi_{b}^{-\infty, \mathcal{E}^{\prime}}(X)\right)$, where $\mathcal{E}^{\prime}=\left(E_{l b}, E_{r b}, E_{f f}\right)$ (see (3.5) for the definition of the space $\Psi_{b}^{-\infty, \mathcal{E}^{\prime}}(X)$ ). Thus, only near $f$, where $\lambda=\infty$ and $\rho=0$, where $\rho$ (as always) is a boundary defining function for $f f\left(X_{b}^{2}\right)$, does $K_{A}$ differ from just a parameter-dependent kernel of a $b$-pseudodifferential operator.

We now define the "residual operators": If $\mathcal{E}=\left(E_{l b}, E_{r b}\right)$ is an index family on $X^{2}$, then we define

$$
\Psi_{\Lambda}^{-\infty, \mathcal{E}}(X):=S^{-\infty}\left(\Lambda ; \Psi^{-\infty, \mathcal{E}}(X)\right) .
$$

See (3.3) for the definition of the space $\Psi^{-\infty, \mathcal{E}}(X)$. We may also define this space as

$$
A \in \Psi_{\Lambda}^{-\infty, \mathcal{E}}(X) \quad \Longleftrightarrow \quad K_{A}=k \nu^{\prime}, \quad k \in \mathcal{A}_{p h g}^{\varnothing, \mathcal{E}}\left(\bar{\Lambda} \times X^{2}\right),
$$

where $\varnothing$ is associated to $\partial_{\infty} \bar{\Lambda}$. 
The full calculus of tempered cone pseudodifferential operators is the sum

$$
\widetilde{\Psi}_{c, \Lambda}^{m, d, \mathcal{E}}(X):=\Psi_{c, \Lambda}^{m, d}(X)+\Psi_{c, \Lambda}^{-\infty, d, \mathcal{E}}(X)+\Psi_{\Lambda}^{-\infty, E_{l b}, E_{r b}}(X) .
$$

The following lemma relates the small calculus and the residual operators to Definition 3.

Lemma 5.1. We have

$$
\Psi_{c, \Lambda}^{-\infty, d}(X) \subseteq \Psi_{c, \Lambda}^{-\infty, d, \mathcal{F}}(X)
$$

where $\mathcal{F}$ is the index family on $\mathcal{T}_{d}$ given by $\mathcal{F}=\left(\varnothing, \varnothing, \mathbb{N}_{0}, \mathbb{N}_{0}\right)$. For any index set $\mathcal{E}=\left(E_{l b}, E_{r b}\right)$, we have

$$
\Psi_{\Lambda}^{-\infty, \mathcal{E}}(X) \subseteq \Psi_{c, \Lambda}^{-\infty, d, \mathcal{E}^{\prime}}(X)
$$

where $\mathcal{E}^{\prime}$ is the index family on $\mathcal{T}_{d}$ given by $\mathcal{E}^{\prime}=\left(E_{l b}, E_{r b}, E_{l b}+E_{r b}, \varnothing\right)$.

Proof. Let $A \in \Psi_{c, \Lambda}^{-\infty, d}(X)$. Then by (1) of Definition $2, K_{A}=R\left(\rho^{d} \lambda\right)$, where $R(\lambda) \in S^{-\infty}\left(\Lambda ; \Psi_{b}^{-\infty}(X)\right)$. Near $f f\left(X_{b}^{2}\right)$, we may assume that $X_{b}^{2} \cong[0,1)_{\rho} \times[-1,1]_{w} \times Y^{2}$, where $\{w=-1\}=l b$ and $\{w=1\}=r b$ (see Figure 1 for a picture of $X_{b}^{2}$ ). Writing $R$ in these coordinates, we have $R=R\left(\rho^{d} \lambda, \rho, w, y, y^{\prime}\right) \nu^{\prime}$, where $R\left(\lambda, \rho, w, y, y^{\prime}\right)$ is a smooth function, vanishing to infinite order at $\lambda=\infty$, and at $w= \pm 1$. If $r=|\mu|$ and $v=\rho / r$, where $\mu=1 / \lambda^{1 / d}$ for $\lambda \in \Lambda$, then lifting $R$ to $\mathcal{T}_{d}$, we find that $R=R\left(v^{d} \omega^{-d}, r v, w, y, y^{\prime}\right) \nu^{\prime}$, where $\omega=\mu /|\mu|$. Now the asymptotic properties of $R\left(\lambda, \rho, w, y, y^{\prime}\right)$ imply that $R\left(v^{d} \omega^{-d}, r v, w, y, y^{\prime}\right)$ has expansions at $l b, r b, v=0, r=0$, and $v=\infty$, with index sets $\varnothing, \varnothing, \mathbb{N}_{0}, \mathbb{N}_{0}$, and $\varnothing$, respectively. Thus, the first statement of this lemma is proved.

Observe that the second statement follows from the definition (5.3).

\subsection{Mapping properties.}

We now describe some mapping properties of the full calculus. We define

$$
X_{d}:=\left[\bar{\Lambda}_{d} \times X ; \partial_{\infty} \bar{\Lambda}_{d} \times Y\right]
$$

Figure 6 gives a pictorial representation of $X_{d}$. If $\beta$ : $X_{d} \rightarrow \bar{\Lambda}_{d} \times X$ is the blow-down map, then we define the following faces in $X_{d}$ : $b x:=$ $\beta^{*}\left(\bar{\Lambda}_{d} \times Y\right)$, boundary of $\mathrm{X} ; f i:=\beta^{*}\left(\partial_{\infty} \bar{\Lambda}_{d} \times Y\right)$, face at infinity; and $b i:=\beta^{*}\left(\partial_{\infty} \bar{\Lambda}_{d} \times X\right)$, boundary at infinity. Before presenting the following two propositions, recall that if $E$ and $F$ are index sets, then $E \bar{\cup} F:=$ $E \cup F \cup\{(z, k+\ell+1) \mid(z, k) \in E,(z, \ell) \in F\}$. 


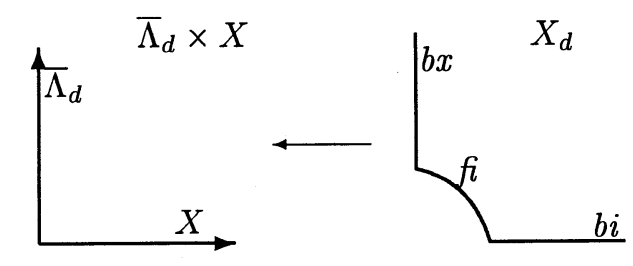

Figure 6: The manifold $X_{d}$. The arrow represents the blow-down map

\section{Proposition 5.2.}

(A) If $f: \Lambda \times X \longrightarrow \Lambda \times X$ is the map $f(\lambda, p)=\left(x(p)^{d} \lambda, p\right)$ and $F$ is any index set, then any $A \in \Psi_{c, \Lambda}^{m, d}(X)$ defines a continuous map

$$
A: \mathcal{A}_{p h g}^{F}(X) \longrightarrow f^{*} S_{c \ell}^{m / d}\left(\Lambda ; \mathcal{A}_{p h g}^{F}(X)\right) \subseteq \mathcal{A}_{p h g}^{\mathcal{G}}\left(X_{d}\right),
$$

where $\mathcal{G}=\left(G_{b x}, G_{f i}, G_{b i}\right)=(F, F,-m)$.

(B) If $A \in \Psi_{c, \Lambda}^{-\infty, d, \mathcal{E}}(X)$ and $F$ is an index set with $E_{r b}+F>0$, then $A$ defines a continuous map

$$
A: \mathcal{A}_{p h g}^{F}(X) \longrightarrow \mathcal{A}_{p h g}^{\mathcal{G}}\left(X_{d}\right),
$$

where $\mathcal{G}=\left(G_{b x}, G_{f i}, G_{b i}\right)=\left(E_{l b} \bar{\cup}\left(E_{f f}+F\right), E_{f i}+F, \varnothing\right)$.

(C) If $A \in \Psi_{\Lambda}^{-\infty, E_{l b}, E_{r b}}(X)$ and $E_{r b}+F>0$, then

$$
A: \mathcal{A}_{p h g}^{F}(X) \longrightarrow S^{-\infty}\left(\Lambda ; \mathcal{A}_{p h g}^{E_{l b}}(X)\right) \subseteq \mathcal{A}_{p h g}^{\mathcal{G}}\left(X_{d}\right),
$$

where $\mathcal{G}=\left(G_{b x}, G_{f i}, G_{b i}\right)=\left(E_{l b}, \varnothing, \varnothing\right)$.

Proof. One can check that $f^{*} S_{c \ell}^{m / d}\left(\Lambda ; \mathcal{A}_{\text {phg }}^{F}(X)\right) \subseteq \mathcal{A}_{\text {phg }}^{\mathcal{G}}\left(X_{d}\right)$, where $\mathcal{G}=$ $(F, F,-m)$. Thus, $(A)$ follows from $(C)$ of Proposition 4.2. The proof of $(B)$ is similar to the proof of $(C)$ of Proposition 4.2 with only more complicated notation due to the slightly more complicated structure of the kernels in $\Psi_{c, \Lambda}^{-\infty, d, \mathcal{E}}(X)$. As the proofs are so similar, we omit the details of (B). Finally, if $A \in \Psi_{\Lambda}^{-\infty, E_{l b}, E_{r b}}(X)$, then $(C)$ follows from the definition (5.2) of the space $\Psi_{\Lambda}^{-\infty, E_{l b}, E_{r b}}(X)$ and the mapping property (3.4).

Combining $(A),(B)$, and $(C)$ of Proposition 5.2 imply the following mapping property of the full calculus. 
Proposition 5.3. If $A \in \widetilde{\Psi}_{c, \Lambda}^{m, d, \mathcal{E}}(X)$ and $F$ is an index set, then provided that $E_{r b}+F>0, A$ defines a continuous map

$$
A: \mathcal{A}_{p h g}^{F}(X) \longrightarrow \mathcal{A}_{p h g}^{\mathcal{G}}\left(X_{d}\right),
$$

where $\mathcal{G}=\left(G_{b x}, G_{f i}, G_{b i}\right)=\left(E_{l b} \bar{\cup}\left(E_{f f}+F\right), E_{f i}+F,-m\right)$.

\subsection{Composition.}

Given $\mathcal{E}=\left(E_{l b}, E_{r b}, E_{f f}, E_{f i}\right)$ and $\mathcal{F}=\left(F_{l b}, F_{r b}, F_{f f}, F_{f i}\right)$, we define the index family $\mathcal{E} \hat{o} \mathcal{F}$ as follows: if $\mathcal{G}=\mathcal{E} \hat{o} \mathcal{F}$, then

$$
\begin{gathered}
G_{l b}=E_{l b} \bar{\cup}\left(E_{f f}+F_{l b}\right) ; G_{r b}=\left(E_{r b}+F_{f f}\right) \bar{\cup} F_{r b} \\
G_{f f}=\left(E_{f f}+F_{f f}\right) \bar{\cup}\left(E_{l b}+F_{r b}\right), \quad \text { and } G_{f i}=E_{f i}+F_{f i} .
\end{gathered}
$$

We begin with the following composition result.

Proposition 5.4. Provided that $E_{r b}+F_{l b}>0$, we have

$$
\Psi_{c, \Lambda}^{-\infty, d, \mathcal{E}}(X) \circ \Psi_{c, \Lambda}^{-\infty, d, \mathcal{F}}(X) \subseteq \Psi_{c, \Lambda}^{-\infty, d, \mathcal{E} \hat{\mathcal{F}} \mathcal{F}}(X) .
$$

If $m, \alpha \in \mathbb{R}$, then

$$
x^{\alpha} \Psi_{c, \Lambda}^{m, d}(X) \circ \Psi_{c, \Lambda}^{-\infty, d, \mathcal{F}}(X) \subseteq \Psi_{c, \Lambda}^{-\infty, d, \mathcal{G}}(X),
$$

where $\mathcal{G}=\left(\alpha+F_{l b}, F_{r b}, \alpha+F_{f f}, \alpha+F_{f i}\right)$; and

$$
\Psi_{c, \Lambda}^{-\infty, d, \mathcal{E}}(X) \circ x^{\alpha} \Psi_{c, \Lambda}^{m, d}(X) \subseteq \Psi_{c, \Lambda}^{-\infty, d, \mathcal{G}}(X),
$$

where $\mathcal{G}=\left(E_{l b}, E_{r b}+\alpha, E_{f f}+\alpha, E_{f i}+\alpha\right)$.

Proof. The proof of this proposition is similar to the proofs of Lemma 4.3 and Theorem 4.4 with only more complicated notation due to the slightly more complicated structure of the kernels in $\Psi_{c, \Lambda}^{-\infty, d, \mathcal{E}}(X)$. As the proofs are so similar, we omit the details of this proof.

We now prove composition involving the residual operators. 


\section{Proposition 5.5.}

(A) If $m, \alpha \in \mathbb{R}$, then

$$
x^{\alpha} \Psi_{c, \Lambda}^{m, d}(X) \circ \Psi_{\Lambda}^{-\infty, \mathcal{F}}(X) \subseteq \Psi_{\Lambda}^{-\infty, \mathcal{G}}(X),
$$

where $\mathcal{G}=\left(\alpha+F_{l b}, F_{r b}\right) ;$ and

$$
\Psi_{\Lambda}^{-\infty, \mathcal{E}}(X) \circ x^{\alpha} \Psi_{c, \Lambda}^{m, d}(X) \subseteq \Psi_{\Lambda}^{-\infty, \mathcal{G}}(X)
$$

where $\mathcal{G}=\left(E_{l b}, E_{r b}+\alpha\right)$.

(B) Provided that $E_{r b}+F_{l b}>0$,

$$
\Psi_{c, \Lambda}^{-\infty, d, \mathcal{E}}(X) \circ \Psi_{\Lambda}^{-\infty, \mathcal{F}}(X) \subseteq \Psi_{\Lambda}^{-\infty, \mathcal{G}}(X),
$$

where $G_{l b}=E_{l b} \bar{\cup}\left(E_{f f}+F_{l b}\right), G_{r b}=F_{r b}$.

Provided that $F_{r b}+E_{l b}>0$,

$$
\Psi_{\Lambda}^{-\infty, \mathcal{F}}(X) \circ \Psi_{c, \Lambda}^{-\infty, d, \mathcal{E}}(X) \subseteq \Psi_{\Lambda}^{-\infty, \mathcal{G}}(X),
$$

where $G_{l b}=F_{l b}, G_{r b}=\left(F_{r b}+E_{f f}\right) \bar{\cup} E_{r b}$.

(C) Finally, provided that $E_{r b}+F_{l b}>0$,

$$
\Psi_{\Lambda}^{-\infty, \mathcal{E}}(X) \circ \Psi_{\Lambda}^{-\infty, \mathcal{F}}(X) \subseteq \Psi_{\Lambda}^{-\infty, \mathcal{G}}(X)
$$

where $G_{l b}=E_{l b}, G_{r b}=F_{r b}$.

Proof. Statement $(C)$ follows from the definition (5.2) and the mapping property (3.4). By taking adjoints, it suffices to prove only the first statements in properties $(A)$ and $(B)$.

Consider the first statement in $(A)$. Let $A=x^{\alpha} B$, where $B \in \Psi_{c, \Lambda}^{m, d}(X)$. Then by $(A)$ of Proposition 5.2, if $f: \Lambda \times X \longrightarrow \Lambda \times X$ is the map $f(\lambda, p)=$ $\left(x(p)^{d} \lambda, p\right)$ and $E$ is any index set on $X$, then $B$ defines a continuous linear map

$$
B: \mathcal{A}_{p h g}^{E}(X) \longrightarrow f^{*} S_{c \ell}^{m / d}\left(\Lambda ; \mathcal{A}_{p h g}^{E}(X)\right) .
$$

Let $C \in \Psi_{\Lambda}^{-\infty, \mathcal{F}}(X)$. Then the kernel of $C$ is a function $C(\lambda, p, q)$ on $\Lambda \times X \times$ $X$ that vanishes to infinite order as $\lambda \rightarrow \infty$ in $\Lambda$, and has expansions as $p$ and $q$ approach the boundary of $X$ with index sets $E_{l b}$ and $E_{r b}$ respectively. Thus, 
as the kernel of $A C$ is just $x^{\alpha} B$ acting on the variable $p$ of $C(\lambda, p, q)$, by (5.7), it follows that $A C \in x^{\alpha} \Psi_{\Lambda}^{-\infty, \mathcal{F}}(X)=\Psi_{\Lambda}^{-\infty, \mathcal{G}}(X)$, where $\mathcal{G}=\left(\alpha+F_{l b}, F_{\text {rb }}\right)$. The first statement is thus proved.

The proof of the first statement in $(B)$ is proved similarly, but here we use the mapping property $(B)$ of Proposition 5.2.

For any index set $\mathcal{G}=\left(G_{l b}, G_{r b}, G_{f f}, G_{f i}\right)$, we define

$$
\widehat{\Psi}_{c, \Lambda}^{-\infty, d, \mathcal{G}}(X):=\Psi_{c, \Lambda}^{-\infty, d, \mathcal{G}}(X)+\Psi_{\Lambda}^{-\infty, \mathcal{G}^{\prime}}(X), \quad \mathcal{G}^{\prime}=\left(G_{l b}, G_{r b}\right) .
$$

Thus, $\widetilde{\Psi}_{c, \Lambda}^{m, d, \mathcal{E}}(X)=\Psi_{c, \Lambda}^{m, d}(X)+\widehat{\Psi}_{c, \Lambda}^{-\infty, d, \mathcal{E}}(X)$. Then Propositions 5.4 and 5.5 yield the following result.

Proposition 5.6. We have

$$
\begin{aligned}
& x^{\alpha} \Psi_{c, \Lambda}^{m, d}(X) \circ \widehat{\Psi}_{c, \Lambda}^{-\infty, d, \mathcal{F}}(X) \subseteq \widehat{\Psi}_{c, \Lambda}^{-\infty, d, \mathcal{G}_{1}}(X) \\
& \widehat{\Psi}_{c, \Lambda}^{-\infty, d, \mathcal{E}}(X) \circ x^{\alpha} \Psi_{c, \Lambda}^{m, d}(X) \subseteq \widehat{\Psi}_{c, \Lambda}^{-\infty, d, \mathcal{G}_{2}}(X),
\end{aligned}
$$

where $\mathcal{G}_{1}=\left(F_{l b}+\alpha, F_{r b}, F_{f f}+\alpha, F_{f i}+\alpha\right), \mathcal{G}_{2}=\left(E_{l b}, E_{r b}+\alpha, E_{f f}+\alpha, E_{f i}+\alpha\right)$. Moreover,

$$
\widehat{\Psi}_{c, \Lambda}^{-\infty, d, \mathcal{E}}(X) \circ \widehat{\Psi}_{c, \Lambda}^{-\infty, d, \mathcal{F}}(X) \subseteq \widehat{\Psi}_{c, \Lambda}^{-\infty, d, \mathcal{E} \hat{\circ} \mathcal{F}}(X),
$$

where $\mathcal{E} \hat{o} \mathcal{F}$ is defined in (5.6).

The following theorem combines the previous propositions.

Theorem 5.7. If $m, m^{\prime} \in \mathbb{R}$, then provided that $E_{r b}+F_{l b}>0$,

$$
\begin{aligned}
\widetilde{\Psi}_{c, \Lambda}^{m, d, \mathcal{E}}(X) \circ \widetilde{\Psi}_{c, \Lambda}^{m^{\prime}, d, \mathcal{F}}(X) \subseteq \Psi_{c, \Lambda}^{m+m^{\prime}, d}(X)+ \\
\widehat{\Psi}_{c, \Lambda}^{-\infty, d, \mathcal{E}}(X)+\widehat{\Psi}_{c, \Lambda}^{-\infty, d, \mathcal{F}}(X)+\widehat{\Psi}_{c, \Lambda}^{-\infty, d, \mathcal{E}} \hat{\circ} \mathcal{F}(X),
\end{aligned}
$$

where $\mathcal{E} \hat{o} \mathcal{F}$ is defined in (5.6).

Proof. If $A \in \widetilde{\Psi}_{c, \Lambda}^{m, d, \mathcal{E}}(X)$ and $B \in \widetilde{\Psi}_{c, \Lambda}^{m^{\prime}, d, \mathcal{F}}(X)$, then we can decompose $A=A_{1}+A_{2}$ and $B=B_{1}+B_{2}$, where $A_{1} \in \Psi_{c, \Lambda}^{m, d}(X), A_{2} \in \widehat{\Psi}_{c, \Lambda}^{-\infty, d, \mathcal{E}}(X)$, $B_{1} \in \Psi_{c, \Lambda}^{m^{\prime}, d}(X)$, and $B_{2} \in \widehat{\Psi}_{c, \Lambda}^{-\infty, d, \mathcal{F}}(X)$. Thus,

$$
A B=A_{1} B_{1}+\left(A_{1} B_{2}+A_{2} B_{1}\right)+A_{2} B_{2} .
$$

By Theorem 4.4, $A_{1} B_{1} \in \Psi_{c, \Lambda}^{m+m^{\prime}, d}(X)$. By Proposition 5.6, it follows that $A_{1} B_{2}+A_{2} B_{1} \in \widehat{\Psi}_{c, \Lambda}^{-\infty, d, \mathcal{E}}(X)+\widehat{\Psi}_{c, \Lambda}^{-\infty, d, \mathcal{F}}(X)$ and that $A_{2} B_{2} \in \widehat{\Psi}_{c, \Lambda}^{-\infty, d, \mathcal{E} \hat{\circ} \mathcal{F}}(X)$. Thus, our theorem is proved. 


\section{The Resolvent as a Tempered operator.}

We begin by defining "cone" Sobolev spaces based on polar coordinates on $\mathbb{R}^{n}$. Thus, let $\left(\mathbb{S}^{n-1}\right)^{\wedge}:=[0, \infty)_{s} \times \mathbb{S}^{n-1}$ be the usual polar coordinates decomposition of $\mathbb{R}^{n}$, with the "blow-down map", cf. Section 2.2 , $\left(\mathbb{S}^{n-1}\right)^{\wedge} \ni$ $(s, \omega) \mapsto s \omega \in \mathbb{R}^{n}$ identifying the interior of $\left(\mathbb{S}^{n-1}\right)^{\wedge}$ with $\mathbb{R}^{n} \backslash\{0\}$. Let $\chi \in C_{c}^{\infty}([0, \infty))$ with $\chi(s) \equiv 1$ near $s=0$. Given $p \in \mathbb{R}$, we define the Sobolev space $H_{c}^{p}\left(\left(\mathbb{S}^{n-1}\right)^{\wedge}\right)$ as those distributions $u$ on $\left(\mathbb{S}^{n-1}\right)^{\wedge}$ such that

$$
u \in H_{c}^{p}\left(\left(\mathbb{S}^{n-1}\right)^{\wedge}\right) \Longleftrightarrow\left\{\begin{array}{l}
\chi(s) u \in H_{b}^{p}\left(\left(\mathbb{S}^{n-1}\right)^{\wedge}\right) \\
(1-\chi(s)) u \in H^{p}\left(\mathbb{R}^{n}\right) .
\end{array}\right.
$$

The statement that $(1-\chi(s)) u \in H^{p}\left(\mathbb{R}^{n}\right)$ uses the identification of $\mathbb{R}^{n} \backslash\{0\}$ with the interior of $\left(\mathbb{S}^{n-1}\right)^{\wedge}$. Thus, $H_{c}^{*}\left(\left(\mathbb{S}^{n-1}\right)^{\wedge}\right)$ interpolates between $b$ Sobolev spaces near $s=0$ and the usual Sobolev spaces outside of $s=0$.

Let $Y^{\wedge}:=[0, \infty)_{s} \times Y$, where $Y=\partial X$. Then appealing to local coordinates on $Y$, we can define $H_{c}^{p}\left(Y^{\wedge}\right)$ for any $p \in \mathbb{R}$. If $A \in x^{-m} \operatorname{Diff}_{b}^{m}(X)$ is written $A=x^{-m} \sum_{k=0}^{m} P_{m-k}(x)\left(x D_{x}\right)^{k}$ near $Y$, where $\bar{P}_{m-k}(x)$ is a differential operator on $Y$ of degree $m-k$, then we define

$$
I(A):=s^{-m} \sum_{k=0}^{m} P_{m-k}(0)\left(s D_{s}\right)^{k} .
$$

Let $\varrho \in C^{\infty}([0, \infty))$, with $\varrho(s)=s$ for $s \leq 1, \varrho>0$ for $1 \leq s \leq 2$, and $\varrho(s) \equiv$ 1 for $s \geq 2$. Then for any $\alpha, p \in \mathbb{R}, I(A): \varrho^{\alpha} H_{c}^{p}\left(Y^{\wedge}\right) \longrightarrow \varrho^{\alpha-m} H_{c}^{p-m}\left(Y^{\wedge}\right)$ continuously. The operator $I(A)$ is called the indicial operator of $A$.

Definition 4. An operator $A \in x^{-m} \operatorname{Diff}_{b}^{m}(X)$ is said to be fully elliptic with respect to $\alpha \in \mathbb{R}$ on a closed sector $\Lambda$ if

1. ${ }^{b} \sigma_{m}\left(x^{m} A\right)(\xi)-\lambda$ is invertible for all $\xi \neq 0$ and $\lambda \in \Lambda$;

2. $\alpha \notin-\operatorname{Im} \operatorname{spec}_{c}(A)$;

3. for any $p \in \mathbb{R}, I(A)-\lambda$ : $\varrho^{\alpha} H_{c}^{p}\left(Y^{\wedge}\right) \longrightarrow \varrho^{\alpha-m} H_{c}^{p-m}\left(Y^{\wedge}\right)$ is invertible for all $\lambda \in \Lambda$ sufficiently large.

Remark 2. In [15], full-ellipticity is called "parameter-ellipticity", and the space $\varrho^{\alpha} H_{c}^{p}\left(Y^{\wedge}\right)$ is denoted by $\mathcal{K}^{p, \alpha+n / 2}\left(Y^{\wedge}\right)$.

The following is the main result of this paper (cf. Theorem 3.2). 
Theorem 6.1. Let $A \in x^{-m} \operatorname{Diff}_{b}^{m}(X)$ be fully elliptic with respect to $\alpha \in \mathbb{R}$ on a closed sector $\Lambda$. Then, $\mathbb{C} \ni \lambda \mapsto(A-\lambda)^{-1}$ is meromorphic with values in $x^{m} \widetilde{\Psi}_{b}^{-m, \mathcal{E}(\alpha)}(X)$ having only finite rank singularities. Here, $\mathcal{E}(\alpha)$ is given in (3.8). Moreover, for all $\lambda \in \Lambda$ sufficiently large,

$$
(A-\lambda)^{-1} \in x^{m} \widetilde{\Psi}_{c, \Lambda}^{-m, m, \mathcal{E}(\alpha), \mathbb{N}_{0}}(X) .
$$

To prove this theorem, we first take a closer look at $I(A)$.

\subsection{Scaling properties of $I(A)$.}

Henceforth, we will use the letter " $x$ " to denote both the boundary defining function on $X$ and the coordinate variable on $[0, \infty)$ in $Y^{\wedge}$. However, it will always be clear which $x$ we are referring to in any given context.

For each $t>0$, define $f_{t}: \Lambda \times Y^{\wedge} \longrightarrow \Lambda \times Y^{\wedge}$ by $f_{t}(\lambda, x, y):=$ $\left(t^{-m} \lambda, t x, y\right)$. Then, $f_{t}$ is a diffeomorphism on $\Lambda \times Y^{\wedge}$ for each $t>0$ with inverse $f_{t}^{-1}(\lambda, x, y)=\left(t^{m} \lambda, t^{-1} x, y\right)$.

Let $\dot{C}^{\infty}\left(\Lambda \times Y^{\wedge}\right)$ be the space of smooth functions on $\Lambda \times Y^{\wedge}$ that vanish to infinite order as $\lambda \rightarrow \infty$ in $\Lambda$, as $x \rightarrow \infty$, and at $\partial\left(\Lambda \times Y^{\wedge}\right)$. Let $C^{-\infty}\left(\Lambda \times Y^{\wedge}\right)$ be its dual. Then given any operator $T$ on $C^{-\infty}\left(\Lambda \times Y^{\wedge}\right)$, we can define the pullback $f_{t}^{*} T$ via $\left(f_{t}^{*} T\right) u:=f_{t}^{*}\left(T\left(f_{t}^{-1}\right)^{*} u\right)$ for all $u \in$ $C^{-\infty}\left(\Lambda \times Y^{\wedge}\right)$. Observe that if $T$ and $S$ are operators on $C^{-\infty}\left(\Lambda \times Y^{\wedge}\right)$, then $f_{t}^{*}(T \circ S)=f_{t}^{*} T \circ f_{t}^{*} S$. In particular,

$$
f_{t}^{*}\left(T^{-1}\right)=\left(f_{t}^{*} T\right)^{-1} \quad \text { if } T^{-1} \text { exists. }
$$

Let $T: \dot{C}^{\infty}\left(Y^{\wedge}\right) \longrightarrow C^{-\infty}\left(\Lambda \times Y^{\wedge}\right)$ be continuous. Then recall that the (Schwartz) kernel of $T, K_{T} \in C^{-\infty}\left(\Lambda \times\left(Y^{\wedge}\right)^{2}\right)$, satisfies

$\left\langle K_{T} ; \psi \otimes \varphi>=\left\langle T \varphi, \psi>, \quad\right.\right.$ for all $\varphi \in \dot{C}^{\infty}\left(Y^{\wedge}\right), \psi \in \dot{C}^{\infty}\left(\Lambda \times Y^{\wedge}\right)$,

where $\psi \otimes \varphi\left(\lambda, x, x^{\prime}, y, y^{\prime}\right)=\psi(\lambda, x, y) \varphi\left(x^{\prime}, y^{\prime}\right)$.

Define

$$
\tilde{f}_{t}: \Lambda \times\left(Y^{\wedge}\right)^{2} \longrightarrow \Lambda \times\left(Y^{\wedge}\right)^{2} \text { by } \tilde{f}_{t}\left(\lambda, x, x^{\prime}, y, y^{\prime}\right):=\left(t^{-m} \lambda, t x, t x^{\prime}, y, y^{\prime}\right) .
$$

Given any continuous linear map $T: \dot{C}^{\infty}\left(Y^{\wedge}\right) \longrightarrow C^{-\infty}\left(\Lambda \times Y^{\wedge}\right)$, a definition chase shows:

If $K_{T}$ is the kernel of $T$, then the kernel of $f_{t}^{*} T$ is $\tilde{f}_{t}^{*} K_{T}$. 
Proposition 6.2. Let $A \in x^{-m} \operatorname{Diff}_{b}^{m}(X)$ be fully elliptic with respect to $\alpha \in \mathbb{R}$ on a closed sector $\Lambda$. Then, identifying $(I(A)-\lambda)^{-1}$ with its kernel on $\Lambda \times\left(Y^{\wedge}\right)^{2}$, we have

$$
\tilde{f}_{t}^{*}(I(A)-\lambda)^{-1}=t^{m}(I(A)-\lambda)^{-1} .
$$

Proof. Let $\varphi \in C^{\infty}\left(\Lambda \times Y^{\wedge}\right)$. Then observe that

$$
\left.x D_{x}\left(f_{t}^{-1}\right)^{*} \varphi=x D_{x}\left(\varphi\left(t^{m} \lambda, t^{-1} x, y\right)\right)=x / t\left(D_{x} \varphi\right)\left(t^{m} \lambda, t^{-1} x, y\right)\right) .
$$

Thus, $x D_{x}\left(f_{t}^{-1}\right)^{*}=\left(f_{t}^{-1}\right)^{*} x D_{x}$. Since $f_{t}^{*}\left(x^{-m}\right)=t^{-m} x^{-m}$ and the indicial operator of $A$ is of the form $I(A)=x^{-m} \sum_{k=1}^{m} P_{m-k}\left(x D_{x}\right)^{k}$, where the $P_{m-k}$ are differential operators on $Y$, it follows that $f_{t}^{*} I(A)=t^{-m} I(A)$. In particular, $f_{t}^{*}(I(A)-\lambda)=t^{-m}(I(A)-\lambda)$, and thus, formula (6.1) yields

$$
f_{t}^{*}(I(A)-\lambda)^{-1}=t^{m}(I(A)-\lambda)^{-1} .
$$

Then (6.3) finishes the proof of this proposition.

If $\mu=1 / \lambda^{1 / m}$, then observe that $\tilde{f}_{t}\left(\mu, x, x^{\prime}, y, y^{\prime}\right)=\left(t \mu, t x, t x^{\prime}, y, y^{\prime}\right)$. Let $\mathcal{T}_{m}\left(Y^{\wedge}\right)$ be the manifold (5.1) for $X=Y^{\wedge}$. Consider now the map $\tilde{f}_{t}$ as a map on $\mathcal{T}_{m}\left(Y^{\wedge}\right)$. Thus, let $\rho=\left|\left(x, x^{\prime}\right)\right|$, and $\theta=\left(x, x^{\prime}\right) /\left|\left(x, x^{\prime}\right)\right|$. Then, $\left(\mu, \rho, \theta, y, y^{\prime}\right)$ are coordinates on $\bar{\Lambda}_{m} \times\left(Y^{\wedge}\right)_{b}^{2}$, and

$$
\tilde{f}_{t}\left(\mu, \rho, \theta, y, y^{\prime}\right)=\left(t \mu, t \rho, \theta, y, y^{\prime}\right) .
$$

Let $r=|\mu|, \omega=\mu /|\mu|, \sigma=|(r, \rho)|$, and $\phi=(r, \rho) /|(r, \rho)|$. Then,

$$
\left(\sigma, \phi, \omega, \theta, y, y^{\prime}\right) \text { are coordinates on } \mathcal{T}_{m}\left(Y^{\wedge}\right),
$$

and by (6.4), $\tilde{f}_{t}\left(\sigma, \phi, \omega, \theta, y, y^{\prime}\right)=\left(t \sigma, \phi, \omega, \theta, y, y^{\prime}\right)$. Thus, $\tilde{f}_{t}$ scales in the defining function for the face $f$.

Combining this property of $\tilde{f}_{t}$ with Proposition 6.2 gives the following.

Corollary 6.3. Let $A \in x^{-m} \operatorname{Diff}_{b}^{m}(X)$ be fully elliptic with respect to $\alpha \in \mathbb{R}$ on a closed sector $\Lambda$. Then, identifying $(I(A)-\lambda)^{-1}$ with its kernel on $\mathcal{T}_{m}\left(Y^{\wedge}\right)$, in the coordinates (6.5), we have

$$
(I(A)-\lambda)^{-1}\left(\sigma, \phi, \omega, \theta, y, y^{\prime}\right)=\left(\sigma / \sigma_{0}\right)^{m}(I(A)-\lambda)^{-1}\left(\sigma_{0}, \phi, \omega, \theta, y, y^{\prime}\right),
$$

for $\sigma_{0}>0$ sufficiently small.

This corollary is important because it states that to determine $(I(A)-$ $\lambda)^{-1}$ at any point $\left(\sigma, \phi, \omega, \theta, y, y^{\prime}\right)$ in $\mathcal{T}_{m}\left(Y^{\wedge}\right)$, we just need to know it at the submanifold $\left\{\sigma=\sigma_{0}\right\}$. 


\subsection{Proof of Theorem 6.1 .}

As already mentioned, we will use the letter " $x$ " to denote both the boundary defining function on $X$ and the coordinate variable on $[0, \infty)$ in $Y^{\wedge}$. However, it will always be clear which $x$ we are referring to in any given context.

Before presenting the proof of Theorem 6.1, we review its outline described in Section 1.4 of the introduction. First, writing $A-\lambda=x^{-m}(P-$ $x^{m} \lambda$ ) where $P$ is a $b$-differential operator, we invert the " $b$-part": $P-x^{m} \lambda$. This is done in Lemma 6.4 using the small tempered calculus. Second, we invert $A$ at the boundary. This is done in Lemma 6.5. Finally, we invert the operator $I(A)-\lambda$ in Lemmas 6.7 and 6.8. As mentioned in the introduction, we want to emphasize that the composition properties of our calculus of parameter-dependent operators make the construction of $(A-\lambda)^{-1}$ very similar to the construction of a parametrix for an elliptic $b$-differential operator. The proofs in this section are quite technical and may be omitted without loss of continuity.

Lemma 6.4. Let $A \in x^{-m} \operatorname{Diff}_{b}^{m}(X)$ be fully elliptic with respect to $\alpha \in \mathbb{R}$ on a closed sector $\Lambda$. Then there exist operators $B \in \Psi_{c, \Lambda}^{-m, m}(X)$ and $R \in$ $\Psi_{c, \Lambda}^{-\infty, m}(X)$ such that $(A-\lambda) x^{m} B=\mathrm{Id}-R$.

Proof. Let $a={ }^{b} \sigma\left(A x^{m}\right)$, and let $\mathcal{U}=[0,1)_{x} \times \mathbb{R}_{y}^{n-1}$ be a coordinate patch on $X$ near $Y$, where $\mathbb{R}^{n-1}$ is a coordinate patch on $Y$. Then, $\left(x^{\prime}, y^{\prime}, z\right)$, where $z=\left(\log \left(x / x^{\prime}\right), y-y^{\prime}\right)$ is a coordinate patch on $X_{b}^{2}$ near $\Delta_{b}$. Let $\varphi \in C_{c}^{\infty}(\mathcal{U})$ and let $\psi(z) \in C_{c}^{\infty}\left(\mathbb{R}^{n}\right)$ be such that $\psi(z) \equiv 1$ on a neighborhood of $z=0$. Finally, let $\chi(\lambda, \xi) \in C^{\infty}\left(\Lambda \times \mathbb{R}^{n}\right)$ with $\chi(\lambda, \xi) \equiv 0$ near $(\lambda, \xi)=0$ and $\chi(\lambda, \xi) \equiv 1$ outside a neighborhood of 0 . Define

$$
K_{B}:=\varphi\left(x^{\prime}, y^{\prime}\right) \psi(z) \int e^{i z \cdot \xi} b\left(\left(x^{\prime}\right)^{m} \lambda, x^{\prime}, y^{\prime}, \xi\right) d \xi \cdot \nu^{\prime}
$$

where $b\left(\lambda, x^{\prime}, y^{\prime}, \xi\right)=\chi(\lambda, \xi)\left(a\left(x^{\prime}, y^{\prime}, \xi\right)-\lambda\right)^{-1}$ and $\nu^{\prime}=\left|\frac{d x^{\prime}}{x^{\prime}} d y^{\prime}\right|$. Then, $B \in \Psi_{c, \Lambda}^{-m, m}(X)$ by Lemma 4.1. Observe that since ${ }^{b} \sigma\left(A x^{m}\right)=a$,

$$
(A-\lambda) x^{m} B=\varphi-S, \quad \text { where } S \in \Psi_{c, \Lambda}^{-1, m}(X) .
$$

If $\mathcal{U}$ is a coordinate patch on the interior of $X$, a similar argument shows that given $\varphi \in C_{c}^{\infty}(\mathcal{U})$, there is a $B \in \Psi_{c, \Lambda}^{-m, m}(X)$ such that (6.6) holds.

Let $\left\{\mathcal{U}_{i}\right\}_{i=1}^{N}$ be coordinate patches covering $X$ such that as in (6.6), there exists a $B_{i} \in \Psi_{c, \Lambda}^{-m, m}(X)$ satisfying $(A-\lambda) x^{m} B_{i}=\varphi_{i}-S_{i}$, where $S_{i} \in$ 
$\Psi_{c, \Lambda}^{-1, m}(X)$, and where $\varphi_{i}$ is a smooth function supported in $\mathcal{U}_{i}$. Setting $B:=\sum_{i=1}^{N} B_{i}$ and assuming that the $\varphi_{i}$ form a partition of unity of $X$, we then have $(A-\lambda) x^{m} B=\mathrm{Id}-S$, where $S \in \Psi_{c, \Lambda}^{-1, m}(X)$. By Theorem 4.4, $S^{j} \in \Psi_{c, \Lambda}^{-j, m}(X)$ for each $j$, and thus by Lemma 4.5 , we can choose an $S^{\prime} \in \Psi_{c, \Lambda}^{-1, m}(X)$ such that $S^{\prime} \sim \sum_{j=1}^{\infty} S^{j}$. It follows that if $B^{\prime}:=B\left(\operatorname{Id}+S^{\prime}\right) \in$ $\Psi_{c, \Lambda}^{-m, m}(X)$, then $(A-\lambda) x^{m} B^{\prime}-\operatorname{Id} \in \Psi_{c, \Lambda}^{-\infty, m}(X)$.

Lemma 6.5. Let $A \in x^{-m} \operatorname{Diff}_{b}^{m}(X)$ be fully elliptic with respect to $\alpha \in \mathbb{R}$ on a closed sector $\Lambda$. Then given $R \in \Psi_{c, \Lambda}^{-\infty, m, \mathcal{E}}(X)$, there is a $B \in \Psi_{c, \Lambda}^{-\infty, m, \mathcal{F}}(X)$, where $\mathcal{F}=\left(\widehat{E}^{+}(\alpha) \bar{\cup} E_{l b}, \varnothing, E_{f f}, E_{f i}\right)$ with $\widehat{E}^{+}(\alpha)$ the index set defined in (3.7), such that $(A-\lambda) x^{m} B-R \in \Psi_{c, \Lambda}^{-\infty, m, \mathcal{G}}(X)$, where $\mathcal{G}=\left(\varnothing, E_{r b}, E_{f f}, E_{f i}\right)$.

Proof. On the decomposition $X \cong[0,1)_{x} \times Y$ near the boundary $Y=\partial X$, we can write $A x^{m}=\sum_{k=0}^{m} B_{k}(x)\left(x D_{x}\right)^{k}$, where $B_{k}(x)$ is a differential operator on $Y$ depending smoothly on $x$. Observe that $\left(r, \omega, v, s, y, y^{\prime}\right)$ where $r=|\mu|$ (where $\mu=1 / \lambda^{1 / m}$ for $\lambda \in \Lambda$ ), $\omega=\mu /|\mu|, v=x^{\prime} / r$, and $s=x / x^{\prime}$, are coordinates near $l b$ in $\mathcal{T}_{m}$. Hence, as $x=s x^{\prime}=s r v$ and $\lambda=r^{-m} \omega^{-m}$, for any function $K$ on $\mathcal{T}_{m}$, we have

$$
(A-\lambda) x^{m} K=\left(\sum_{k=0}^{m} B_{k}(s r v)\left(s D_{s}\right)^{k}-s^{m} v^{m} \omega^{-m}\right) K\left(r, \omega, v, s, y, y^{\prime}\right)=\widetilde{A} K,
$$

where $\widetilde{A}=\sum_{k=0}^{m} \widetilde{B}_{k}(r, \omega, v, s)\left(s D_{s}\right)^{k}$, where $\widetilde{B}_{k}(r, \omega, v, s)$ is the following differential operator on $Y$ depending smoothly on $(r, \omega, v, s)$ :

$$
\widetilde{B}_{k}(r, \omega, v, s)=\left\{\begin{array}{l}
B_{0}(s r v)-s^{m} v^{m} \omega^{-m} \quad \text { if } k=0 \\
B_{k}(s r v) \text { if } k>0 .
\end{array}\right.
$$

Now given $R \in \Psi_{c, \Lambda}^{-\infty, m, \mathcal{E}}(X), K_{R}=f\left(r, \omega, v, s, y, y^{\prime}\right) \nu^{\prime}$ has an expansion as $s \downarrow 0$ with index set $E_{l b}$. Hence by [35, Lem. 5.44], there exists a $B \in \Psi_{c, \Lambda}^{-\infty, m, \mathcal{F}}(X)$ where $\mathcal{F}=\left(\widehat{E}^{+}(\alpha) \bar{\cup} E_{l b}, \varnothing, E_{f f}, E_{f i}\right)$, such that if $K_{B}=g \nu^{\prime}$, then $\widetilde{A} g-f$ is the kernel of an operator in $\Psi_{c, \Lambda}^{-\infty, m, \mathcal{G}}(X)$ where $\mathcal{G}=\left(\varnothing, E_{r b}, E_{f f}, E_{f}\right)$. Since the kernel of $(A-\lambda) x^{m} B$ is $\widetilde{A} g \nu^{\prime}$ by (6.7), our lemma is proved.

Our next lemma deals with functions that have only partial expansions. Thus, let $E$ be an index set, $r \in \mathbb{R}, Z$ be a manifold with corners, and 
$H \in M_{1}(Z)$. Then a function $u \in S^{\alpha}(Z)$ (for some $\alpha$ ) has a partial expansion at $H$ with index set $E$ of order $r$, if $u$ satisfies the expansion condition (2.1) with $M=N=r$. If $\mathcal{A} \subseteq M_{1}(Z)$ and $\mathcal{E}$ is an index family associated to $\mathcal{A}$, then $\mathcal{A}_{r}^{\mathcal{E}}(Z)$ is the space of functions $u$ such that for each $H \in \mathcal{A}, u$ has a partial expansion at $H$ with index set $E_{H}$ of order $r$.

Lemma 6.6. Let $R \in \Psi_{c, \Lambda}^{-\infty, m, \mathcal{E}}\left(Y^{\wedge}\right)$ with $E_{f f}=\varnothing$ and $E_{f i} \geq 0$. Then writing $K_{R}=u\left|\frac{d x^{\prime}}{x^{\prime}} d y^{\prime}\right|$, for any $N \in \mathbb{N}_{0}$ and for $\lambda$ away from 0 , we have $u \in S^{2 N+1}\left(\Lambda ; \mathcal{A}_{m N}^{\left(E_{l b}, E_{r b}\right)}\left(\left(Y^{\wedge}\right)^{2}\right)\right)$.

Proof. By the definition of $\Psi_{c, \Lambda}^{-\infty, m, \mathcal{E}}$, we may assume that $u$ is supported near fi. Let $r=|\mu|$ with $\mu=1 / \lambda^{1 / m}$ and let $\rho$ be a boundary defining function for $f f\left(\left(Y^{\wedge}\right)_{b}^{2}\right)$. Then, $\rho / r$ is a boundary defining function for $f f\left(\mathcal{T}_{m}\left(Y^{\wedge}\right)\right)$. Hence, as $u$ vanishes to infinite order at $f f\left(\mathcal{T}_{m}\left(Y^{\wedge}\right)\right)$ (by assumption) and at $b i\left(\mathcal{T}_{m}\left(Y^{\wedge}\right)\right)$ (by definition), for any $k$ we can write $u=r^{-k} \rho^{k} u_{k}=|\lambda|^{k / m} \rho^{k} u_{k}$, where $u_{k}$ has the same polyhomogeneous properties as $u$ on $\mathcal{T}_{m}\left(Y^{\wedge}\right)$.

Choosing $k=2 m N+m$, it suffices to prove that $\rho^{2 m N+m} u_{2 m N+m} \in$ $S^{0}\left(\Lambda ; \mathcal{A}_{m N}^{\left(E_{l l}, E_{r b}\right)}\left(\left(Y^{\wedge}\right)^{2}\right)\right)$. Denote $u_{2 m N+m}$ by $w$. For simplicity, we assume that $w$ is supported near $l b$; the proof is similar if it is supported near $r b$. Thus, in the coordinates $\left(r, \omega, v, s, y, y^{\prime}\right)$, where $\omega=\mu /|\mu|, v=x^{\prime} / r$, and $s=x / x^{\prime}$, near $l b$ in $\mathcal{T}_{m}\left(Y^{\wedge}\right)$, we have

$$
w=\sum_{\operatorname{Re} z \leq m N,(z, k) \in E_{l b}} s^{z}(\log s)^{k} u_{(z, k)}\left(r, \omega, v, y, y^{\prime}\right)+s^{m N} u_{N}\left(r, \omega, v, s, y, y^{\prime}\right),
$$

where since $E_{f f}=\varnothing$ and $E_{f i} \geq 0, u_{(z, k)}, u_{N} \in S^{0}\left(\mathcal{T}_{m}\left(Y^{\wedge}\right)\right)$. Since $s=$ $x / x^{\prime}$, we have $(\log s)^{k}=\sum_{j=0}^{k}\left(\begin{array}{c}k \\ j\end{array}\right)(\log x)^{j}\left(-\log x^{\prime}\right)^{k-j}$. Thus, as $\rho=x^{\prime}$ is a boundary defining function for $f f$ near $l b$, we have

$$
\begin{aligned}
\rho^{2 m N+m} w=\sum_{\operatorname{Re} z \leq m N,(z, k) \in E_{l b}} x^{z} \sum_{j=0}^{k}\left(\begin{array}{c}
k \\
j
\end{array}\right)(\log x)^{j} \widetilde{u}_{(z, j, k)}\left(r, \omega, x^{\prime}, y, y^{\prime}\right) & \\
& +x^{m N} \widetilde{u}_{m N}\left(r, \omega, x, x^{\prime}, y, y^{\prime}\right),
\end{aligned}
$$

with $\widetilde{u}_{(z, j, k)}\left(r, \omega, x^{\prime}, y, y^{\prime}\right)=\left(x^{\prime}\right)^{2 m N+m-z}\left(-\log x^{\prime}\right)^{k-j} u_{(z, k)}\left(r, \omega, x^{\prime} / r, y, y^{\prime}\right)$, and with $\widetilde{u}_{m N}\left(r, \omega, x, x^{\prime}, y, y^{\prime}\right)=\left(x^{\prime}\right)^{m N+m} u_{m N}\left(r, \omega, x^{\prime} / r, x / x^{\prime}, y, y^{\prime}\right)$. Since $\operatorname{Re} z \leq m N$ and $u_{(z, k)}, u_{m N} \in S^{0}\left(\mathcal{T}_{m}\left(Y^{\wedge}\right)\right)$, it follows that $\widetilde{u}_{(z, j, k)}, \widetilde{u}_{m N} \in$ $\left(x^{\prime}\right)^{m N} S^{0}\left(\Lambda \times\left(Y^{\wedge}\right)^{2}\right)$ away from $\lambda=0$. 
Let $A \in x^{-m} \operatorname{Diff}_{b}^{m}(X)$ be fully elliptic with respect to $\alpha \in \mathbb{R}$ on a closed sector $\Lambda$. For the next two lemmas, we will denote

$$
a(\lambda)=(I(A)-\lambda) .
$$

Lemma 6.7. There exists $B \in \widetilde{\Psi}_{c, \Lambda}^{-m, m, \mathcal{E}}\left(Y^{\wedge}\right)$ and $R \in \Psi_{c, \Lambda}^{-\infty, m, \mathcal{F}}\left(Y^{\wedge}\right)$, where $\mathcal{E}=\mathcal{E}(A, \alpha)$ is of the form $\left(E_{l b}, E_{r b}, E_{f f}, \mathbb{N}_{0}\right)$, with $E_{l b}>\alpha-m, E_{r b}>-(\alpha-$ $m), E_{f f}>0$, and where $\mathcal{F}=\left(\varnothing, \widehat{E}^{-}(\alpha), \varnothing, \mathbb{N}_{0}\right)$, such that $a(\lambda) x^{m} B=\mathrm{Id}-R$.

Proof. By Lemma 6.4, there is a $B_{0} \in \Psi_{c, \Lambda}^{-m, m}\left(Y^{\wedge}\right)$ and an $R_{0} \in \Psi_{c, \Lambda}^{-\infty, m}\left(Y^{\wedge}\right)$ such that

$$
a(\lambda) x^{m} B_{0}=\mathrm{Id}-R_{0} .
$$

Let $B_{0}^{\prime}$ be the $b$-pseudodifferential operator with kernel

$$
K_{B_{0}^{\prime}}=\frac{\varphi\left(x, x^{\prime}\right)}{2 \pi} \int_{\operatorname{Im} \tau=-(\alpha-m)}\left(x / x^{\prime}\right)^{i \tau} \widehat{A x^{m}}(\tau)^{-1} \circ \widehat{R_{0}}(\tau) d \tau \cdot\left|\frac{d x^{\prime}}{x^{\prime}} d y^{\prime}\right|,
$$

where $\varphi \in C_{c}^{\infty}\left([0, \infty)^{2}\right)$ with $\varphi \equiv 1$ near $(0,0)$. Then, see [35, Sec. 5.7], $B_{0}^{\prime} \in \Psi_{b}^{-\infty, \mathcal{E}_{0}}\left(Y^{\wedge}\right)$ with $\mathcal{E}_{0}=\left(E_{0, l b}, E_{0, r b}, \mathbb{N}_{0}\right)=\left(E^{+}(\alpha), E^{-}(\alpha), \mathbb{N}_{0}\right)$, where $E^{ \pm}(\alpha)$ are given in (3.6). Let $\psi(\lambda) \in C_{c}^{\infty}(\mathbb{C})$ be such that $\psi(\lambda) \equiv 1$ near $\lambda=0$ and set $B_{1}:=B_{0}+\psi\left(\rho^{m} \lambda\right) B_{0}^{\prime}$, where $\rho=\left|\left(x, x^{\prime}\right)\right|$ is a boundary defining function for $f f\left(\left(Y^{\wedge}\right)_{b}^{2}\right)$. Then by Lemma 5.1, $B_{1} \in \widetilde{\Psi}_{c, \Lambda}^{-m, m, \mathcal{E}_{1}}\left(Y^{\wedge}\right)$, where $\mathcal{E}_{1}$ is of the form $\mathcal{E}_{1}=\left(E_{0, l b}, E_{0, r b}, \mathbb{N}_{0}, \mathbb{N}_{0}\right)$, and it satisfies

$$
a(\lambda) x^{m} B_{1}=\mathrm{Id}-R_{1},
$$

where by the composition properties of tempered operators (Proposition 5.4), $R_{1}=R_{0}-a(\lambda) x^{m} \psi\left(\rho^{m} \lambda\right) B_{0}^{\prime} \in \Psi_{c, \Lambda}^{-\infty, m, \mathcal{F}_{1}}\left(Y^{\wedge}\right)$, where $\mathcal{F}_{1}=\mathcal{E}_{1}$. By (6.8), the normal operator of $a(\lambda) x^{m} \circ \psi\left(\rho^{m} \lambda\right) B_{0}^{\prime}$ is $\widehat{A x^{m}} \circ \widehat{B_{0}^{\prime}}=\widehat{R_{0}}$, and hence, $\widehat{R_{1}}=0$. Thus, $R_{1}$ vanishes to first order at $f f\left(\mathcal{T}_{m}\left(Y^{\wedge}\right)\right)$ and so in fact, $\mathcal{F}_{1}=\left(E_{0, l b}, E_{0, r b}, \mathbb{N}, \mathbb{N}_{0}\right)$.

By Lemma 6.5 , there is a $B_{1}^{\prime} \in \Psi_{c, \Lambda}^{-\infty, m, \mathcal{E}_{1}^{\prime}}\left(Y^{\wedge}\right)$, where $\mathcal{E}_{1}^{\prime}=$ $\left(\widehat{E}^{+}(\alpha) \bar{\cup} E_{0, l b}, \varnothing, \mathbb{N}, \mathbb{N}_{0}\right)$ with $\widehat{E}^{+}(\alpha)$ the index set (3.7), such that

$$
a(\lambda) x^{m} B_{1}^{\prime}-R_{1} \in \Psi_{c, \Lambda}^{-\infty, m, \mathcal{F}_{2}}\left(Y^{\wedge}\right),
$$

where $\mathcal{F}_{2}=\left(\varnothing, E_{0, r b}, \mathbb{N}, \mathbb{N}_{0}\right)$. Let $B_{2}:=B_{1}+B_{1}^{\prime} \in \widetilde{\Psi}_{c, \Lambda}^{-m, m, \mathcal{E}_{2}}\left(Y^{\wedge}\right)$, where $\mathcal{E}_{2}=\mathcal{E}_{1} \cup \mathcal{E}_{1}^{\prime}$. Then by (6.9),

$$
a(\lambda) x^{m} B_{2}=\mathrm{Id}-R_{2},
$$


where $R_{2} \in \Psi_{c, \Lambda}^{-\infty, m, \mathcal{F}_{2}}\left(Y^{\wedge}\right)$.

Observe that for each $j, R_{2}^{j} \in \Psi_{c, \Lambda}^{-\infty, m, \mathcal{F}_{2, j}}\left(Y^{\wedge}\right)$, where the $\mathcal{F}_{2, j}$ are defined inductively using Proposition 5.4 and the fact that $R_{2}^{j+1}=R_{2} \circ R_{2}^{j}$ :

$$
\mathcal{F}_{2,1}:=\mathcal{F}_{2}, \mathcal{F}_{2, j+1}:=\left(\varnothing,\left(E_{0, r b}+j\right) \bar{\cup} F_{2, j, r b}, \mathbb{N}_{j+1}, \mathbb{N}_{0}\right),
$$

where $\mathbb{N}_{j}=\{j, j+1, j+2, \ldots\}$. Since $\widehat{E}^{-}(\alpha)=\bar{\bigcup}_{r \in \mathbb{N}_{0}}\left(E^{-}(\alpha)+r\right)$ and $E_{0, r b}=E^{-}(\alpha)$, it follows that $F_{2, j, r b} \subseteq \widehat{E}^{-}(\alpha)$ for each $j$ and $F_{2, j, r b} \rightarrow \widehat{E}^{-}(\alpha)$ as $j \rightarrow+\infty$ in the sense that $c \in \widehat{E}^{-}(\alpha)$ if and only if for some $j, c \in F_{2, j, r b}$. Hence, we can find an asymptotic sum $R_{2}^{\prime} \sim \sum_{j=1}^{\infty} R_{2}^{j} \in \Psi_{c, \Lambda}^{-\infty, m, \mathcal{F}_{2}^{\prime}}\left(Y^{\wedge}\right)$, where $\mathcal{F}_{2}^{\prime}:=\left(\varnothing, \widehat{E}^{-}(\alpha), \mathbb{N}, \mathbb{N}_{0}\right)$, such that for each $N$,

$$
R_{2}^{\prime}-\sum_{j=1}^{N-1} R_{2}^{j} \in \Psi_{c, \Lambda}^{-\infty, m, \mathcal{F}_{2, N}^{\prime}}\left(Y^{\wedge}\right), \quad \mathcal{F}_{2, N}^{\prime}:=\left(\varnothing, \widehat{E}^{-}(\alpha), \mathbb{N}_{N}, \mathbb{N}_{0}\right)
$$

Thus, setting $B_{3}:=B_{2}\left(\mathrm{Id}+R_{2}^{\prime}\right) \in \widetilde{\Psi}_{c, \Lambda}^{-m, m, \mathcal{E}_{3}}\left(Y^{\wedge}\right)$, where (see Theorem 5.7) $\mathcal{E}_{3}=\mathcal{E}_{2} \cup \mathcal{F}_{2}^{\prime} \cup \mathcal{E}_{2} \hat{\circ} \mathcal{F}_{2}^{\prime}$, it follows that

$$
a(\lambda) x^{m} B_{3}=\mathrm{Id}-R_{3},
$$

where $R_{3} \in \Psi_{c, \Lambda}^{-\infty, m, \mathcal{F}_{3}}\left(Y^{\wedge}\right)$, where $\mathcal{F}_{3}=\left(\varnothing, \widehat{E}^{-}(\alpha), \varnothing, \mathbb{N}_{0}\right)$. Following the construction of $B_{3}$, one can see that $\mathcal{E}_{3}=\left(E_{3, l b}, E_{3, r b}, E_{3, f f}, \mathbb{N}_{0}\right)$, where $E_{3, l b}>\alpha-m, E_{3, r b}>-(\alpha-m)$, and $E_{3, f f}>0$.

Lemma 6.8. For $\lambda$ sufficiently large in $\Lambda, a(\lambda)^{-1} \in x^{m} \widetilde{\Psi}_{c, \Lambda}^{-m, m, \mathcal{E}}\left(Y^{\wedge}\right)$, where $\mathcal{E}=\mathcal{E}(A, \alpha)$ is an index set of the form $\left(E_{l b}, E_{r b}, E_{f f}, \mathbb{N}_{0}\right)$, with $E_{l b}>\alpha-m$, $E_{r b}>-(\alpha-m)$, and $E_{f f}>0$.

Proof. By Lemma 6.7, there is a $B \in \widetilde{\Psi}_{c, \Lambda}^{-m, m, \mathcal{E}}\left(Y^{\wedge}\right)$, where $\mathcal{E}=\mathcal{E}(A, \alpha)$ is an index set of the form $\left(E_{l b}, E_{r b}, E_{f f}, \mathbb{N}_{0}\right)$, with $E_{l b}>\alpha-m, E_{r b}>-(\alpha-m)$, $E_{f f}>0$, and an $R \in \Psi_{c, \Lambda}^{-\infty, m, \mathcal{F}}\left(Y^{\wedge}\right)$, where $\mathcal{F}=\left(\varnothing, \widehat{E}^{-}(\alpha), \varnothing, \mathbb{N}_{0}\right)$, such that

$$
a(\lambda) x^{m} B=\mathrm{Id}-R \text {. }
$$

Let $A=x^{-m} P$, where $P \in \operatorname{Diff}_{b}^{m}(X)$. Then, $A^{t}=x^{-m} P^{\prime}$, where $P^{\prime}=x^{m} P^{t} x^{-m}$. Note that $\widehat{P^{\prime}}(\tau)=\widehat{P^{t}}(\tau+i m)=\widehat{P}(-\tau-i m)^{t}$. Thus, as $-\alpha \notin \operatorname{Imspec}_{c}(A)$, it follows that $-\alpha+m \notin-\operatorname{Im} \operatorname{spec}_{c}\left(A^{t}\right)$. Also, since for any $s \in \mathbb{R}, I(A)-\lambda: \varrho^{\alpha} H_{c}^{s}\left(Y^{\wedge}\right) \longrightarrow \varrho^{\alpha-m} H_{c}^{s-m}\left(Y^{\wedge}\right)$ is an isomorphism 
for all $\lambda \in \Lambda$ large, it follows that for all $s \in \mathbb{R}, I\left(A^{t}\right)-\lambda=(I(A)-$ $\lambda)^{t}: \varrho^{-\alpha+m} H_{c}^{-s+m}\left(Y^{\wedge}\right) \longrightarrow \varrho^{-\alpha} H_{c}^{-s}\left(Y^{\wedge}\right)$ is an isomorphism for all $\lambda \in \Lambda$ large. Thus, $A^{t}$ is fully elliptic with respect to $-\alpha+m$ on $\Lambda$. Thus, applying (6.10) to $a(\lambda)^{t}$ gives

$$
a(\lambda)^{t} x^{m} \widetilde{B}=\mathrm{Id}-\widetilde{R},
$$

where $\widetilde{B} \in \widetilde{\Psi}_{c, \Lambda}^{-m, m, \widetilde{\mathcal{E}}}\left(Y^{\wedge}\right)$ with $\widetilde{\mathcal{E}}=\left(\widetilde{E}_{l b}, \widetilde{E}_{r b}, \widetilde{E}_{f f}, \mathbb{N}_{0}\right)$, where $\widetilde{E}_{l b}>-\alpha$, $\widetilde{E}_{r b}>\alpha, \widetilde{E}_{f f}>0$, and where $\widetilde{R} \in \Psi_{c, \Lambda}^{-\infty, m, \widetilde{\mathcal{F}}}\left(Y^{\wedge}\right)$ with $\widetilde{\mathcal{F}}=\left(\varnothing, \widehat{F}^{+}(\alpha), \varnothing, \mathbb{N}_{0}\right)$, where $\widehat{F}^{+}(\alpha)>\alpha$ is the set $\widehat{E}^{-}(-\alpha+m)$ for $A^{t}$. Taking the transpose of the above equation gives

$$
x^{m} B^{\prime} a(\lambda)=\mathrm{Id}-R^{\prime}
$$

where $B^{\prime}=x^{-m} \widetilde{B}^{t} x^{m} \in \widetilde{\Psi}_{c, \Lambda}^{-m, m, \mathcal{E}^{\prime}}\left(Y^{\wedge}\right), R^{\prime}=\widetilde{R}^{t} \in \Psi_{c, \Lambda}^{-\infty, m, \mathcal{F}^{\prime}}\left(Y^{\wedge}\right)$, where $\mathcal{E}^{\prime}=\left(E_{l b}^{\prime}, E_{r b}^{\prime}, E_{f f}^{\prime}, \mathbb{N}_{0}\right)$, with $E_{l b}^{\prime}=\widetilde{E}_{r b}-m>\alpha-m, E_{r b}^{\prime}=\widetilde{E}_{l b}+m>$ $-(\alpha-m), E_{f f}^{\prime}>0$, and where $\mathcal{F}^{\prime}=\left(\widehat{F}^{+}(\alpha), \varnothing, \varnothing, \mathbb{N}_{0}\right)$.

From (6.10) and (6.11) it follows that $a(\lambda)^{-1}=x^{m} B+a(\lambda)^{-1} R$ and $a(\lambda)^{-1}=x^{m} B^{\prime}+R^{\prime} a(\lambda)^{-1}$. Plugging the second equation into the first gives

$$
a(\lambda)^{-1}=x^{m} B+x^{m} B^{\prime} R+R^{\prime} a(\lambda)^{-1} R .
$$

Let $\varphi \in C^{\infty}\left(\Lambda \times[0, \infty)_{x} \times Y\right)$ have bounded derivatives and vanish for $|\lambda|^{-2}+x^{2}$ small. Then, applying $\varphi$ to the right-hand side of (6.12) yields

$$
a(\lambda)^{-1} \varphi=x^{m} B \varphi+x^{m} B^{\prime} R \varphi+R^{\prime} a(\lambda)^{-1} R \varphi .
$$

Since when $\varphi$ is lifted to the right in $\mathcal{T}_{m}\left(Y^{\wedge}\right)$, it vanishes near the hypersurface $f i$, it follows that $R \varphi \in \Psi_{c, \Lambda}^{-\infty, m,\left(\varnothing, \widehat{E}^{-}(\alpha), \varnothing, \varnothing\right)}\left(Y^{\wedge}\right)$. Also, by Lemma 6.6 , omitting density factors, $R^{\prime} \in S^{2 N+1}\left(\Lambda ; \mathcal{A}_{m N}^{\left(\widehat{F}^{+}(\alpha), \varnothing\right)}\left(\left(Y^{\wedge}\right)^{2}\right)\right)$ for any $N$. Thus, by (6.13), for any $N$,

$$
\begin{aligned}
& a(\lambda)^{-1} \varphi \in x^{m} \widetilde{\Psi}_{c, \Lambda}^{-m, m,\left(E_{l b}, E_{r b}, E_{f f}, \varnothing\right)}\left(Y^{\wedge}\right)+ \\
& x^{m} \Psi_{c, \Lambda}^{-\infty, m,\left(E_{l b}^{\prime}, \widehat{E}^{-}(\alpha), \varnothing, \varnothing\right)}\left(Y^{\wedge}\right)+S^{-\infty}\left(\Lambda ; \mathcal{A}_{m N}^{\left(\widehat{F}^{+}(\alpha), \widehat{E}^{-}(\alpha)\right)}\left(\left(Y^{\wedge}\right)^{2}\right)\right) .
\end{aligned}
$$

Since $N$ is arbitrary, it follows that

$$
\begin{aligned}
& a(\lambda)^{-1} \varphi \in x^{m} \widetilde{\Psi}_{c, \Lambda}^{-m, m,\left(E_{l b}, E_{r b}, E_{f f}, \varnothing\right)}\left(Y^{\wedge}\right)+ \\
& \quad x^{m} \Psi_{c, \Lambda}^{-\infty, m,\left(E_{l b}^{\prime}, \widehat{E}^{-}(\alpha), \varnothing, \varnothing\right)}\left(Y^{\wedge}\right)+\Psi_{\Lambda}^{-\infty, \widehat{F}^{+}(\alpha), \widehat{E}^{-}(\alpha)}\left(Y^{\wedge}\right) .
\end{aligned}
$$


A similar argument multiplying $\varphi$ to the left-hand side of (6.12) yields

$$
\begin{gathered}
\varphi a(\lambda)^{-1} \in x^{m} \widetilde{\Psi}_{c, \Lambda}^{-m, m,\left(E_{l b}, E_{r b}, E_{f}, \varnothing\right)}\left(Y^{\wedge}\right)+ \\
x^{m} \Psi_{c, \Lambda}^{-\infty, m,\left(E_{l b}^{\prime}, \widehat{E}^{-}(\alpha), E_{l b}^{\prime}+\widehat{E}^{-}(\alpha), \varnothing\right)}\left(Y^{\wedge}\right)+\Psi_{\Lambda}^{-\infty, \widehat{F}^{+}(\alpha), \widehat{E}^{-}(\alpha)}\left(Y^{\wedge}\right) .
\end{gathered}
$$

Thus, if $\Phi\left(\lambda, x, x^{\prime}, y, y^{\prime}\right)=\varphi(\lambda, x, y)+\varphi\left(\lambda, x^{\prime}, y^{\prime}\right)$, then adding (6.14) and (6.15) gives

$$
\begin{gathered}
\Phi a(\lambda)^{-1} \in x^{m} \widetilde{\Psi}_{c, \Lambda}^{-m, m,\left(E_{l b}, E_{r b}, E_{f f}, \varnothing\right)}\left(Y^{\wedge}\right)+ \\
x^{m} \Psi_{c, \Lambda}^{-\infty, m,\left(E_{l b}^{\prime}, \widehat{E}^{-}(\alpha), E_{l b}^{\prime}+\widehat{E}^{-}(\alpha), \varnothing\right)}\left(Y^{\wedge}\right)+\Psi_{\Lambda}^{-\infty, \widehat{F}^{+}(\alpha), \widehat{E}^{-}(\alpha)}\left(Y^{\wedge}\right) .
\end{gathered}
$$

Let $F_{l b}=E_{l b} \cup E_{l b}^{\prime} \cup\left(\widehat{F}^{+}(\alpha)-m\right), F_{r b}=E_{r b} \cup E_{r b}^{\prime} \cup \widehat{E}^{-}(\alpha)$, and let $F_{f f}=E_{f f} \cup\left(E_{l b}^{\prime}+\widehat{E}^{-}(\alpha)\right)$. Then observe that $F_{l b}>\alpha-m, F_{r b}>-(\alpha-m)$, and $F_{f f}>0$; and that

$$
\Phi a(\lambda)^{-1} \in x^{m} \widetilde{\Psi}_{c, \Lambda}^{-m, m,\left(F_{l b}, F_{r b}, F_{f f}, \varnothing\right)}\left(Y^{\wedge}\right) .
$$

Now we may choose $\varphi$ such that in the coordinates (6.5), $\Phi>0$ for $\varepsilon / 2<$ $\sigma<2 \varepsilon$ where $\varepsilon$ is sufficiently small so that $a(\lambda)^{-1}$ exists for all $\lambda$ such that $\sigma<2 \varepsilon$. Thus, with $\sigma_{0}=\varepsilon$ in Corollary 6.3, formula (6.16) implies that $a(\lambda)^{-1} \in x^{m} \widetilde{\Psi}_{c, \Lambda}^{-m, m, \mathcal{F}}\left(Y^{\wedge}\right)$, where $\mathcal{F}=\left(F_{l b}, F_{r b}, F_{f f}, \mathbb{N}_{0}\right)$.

We need one more lemma before proving our main theorem.

Lemma 6.9. Let $R \in \Psi_{\Lambda}^{-\infty, \mathcal{E}}(X)$. Then given $s \in \mathbb{R}$, and $\gamma \in \mathbb{R}$ with $E_{l b}>\gamma$ and $E_{r b}>-\gamma$, Id $-R$ is invertible on $x^{\gamma} H_{b}^{s}(X)$ for $\lambda$ sufficiently large. Moreover, $(\operatorname{Id}-R)^{-1}=\operatorname{Id}+S$, where $S \in \Psi_{\Lambda}^{-\infty, \mathcal{E}}(X)$.

Proof. Since $E_{l b}>\gamma$ and $E_{r b}>-\gamma$, by the mapping property (3.4) and the definition of $\Psi_{\Lambda}^{-\infty, \mathcal{E}}(X), R \in S^{-\infty}\left(\Lambda ; \Psi^{-\infty, \mathcal{E}}(X)\right) \subseteq S^{-\infty}\left(\Lambda ; \mathcal{B}\left(x^{\gamma} H_{b}^{s}(X)\right)\right)$, where $\mathcal{B}\left(x^{\gamma} H_{b}^{s}(X)\right)$ is the space of bounded operators on $x^{\gamma} H_{b}^{s}(X)$. The usual geometric series argument now completes the proof.

Proof of Theorem 6.1: By Lemma 6.4, there is a $B_{0} \in \Psi_{c, \Lambda}^{-m, m}(X)$ and $R_{0} \in \Psi_{c, \Lambda}^{-\infty, m}(X)$ such that

$$
(A-\lambda) x^{m} B_{0}=\mathrm{Id}-R_{0} .
$$

By Lemma $6.8,(I(A)-\lambda)^{-1} \in x^{m} \widetilde{\Psi}_{c, \Lambda}^{-m, m, \mathcal{E}}\left(Y^{\wedge}\right)$ for $\lambda \in \Lambda$ sufficiently large, where $\mathcal{E}=\mathcal{E}(A, \alpha)$ is an index set of the form $\left(E_{l b}, E_{r b}, E_{f f}, \mathbb{N}_{0}\right)$, with $E_{l b}>$ 
$\alpha-m, E_{r b}>-(\alpha-m)$, and $E_{f f}>0$. Let $\psi, \psi^{\prime} \in C^{\infty}(X)$ be such that $\psi, \psi^{\prime} \equiv 1$ near $Y$ and such that $\psi^{\prime} \equiv 1$ on $\operatorname{supp} \psi$. Let

$$
B_{0}^{\prime}:=x^{-m} \psi(I(A)-\lambda)^{-1} \psi^{\prime} R_{0} .
$$

Then, $B_{0}^{\prime} \in \widetilde{\Psi}_{c, \Lambda}^{-\infty, m, \mathcal{E}}(X)$. Since near $Y, A=I(A) \bmod x^{-m+1} \operatorname{Diff}_{b}^{m}(X)$, and since $\psi^{\prime} \equiv 1$ on $\operatorname{supp} \psi$, it follows that

$$
(A-\lambda) x^{m} B_{0}^{\prime}=\psi R_{0} \bmod x \widetilde{\Psi}_{c, \Lambda}^{-\infty, m, \mathcal{E}}(X) .
$$

Thus, if $B_{1}:=B_{0}+B_{0}^{\prime} \in \widetilde{\Psi}_{c, \Lambda}^{-m, m, \mathcal{E}_{1}}(X)$, where $\mathcal{E}_{1}=\mathcal{E}$, then

$$
(A-\lambda) x^{m} B_{1}=\mathrm{Id}-R_{1},
$$

where $R_{1} \in \dot{x} \widetilde{\Psi}_{c, \Lambda}^{-\infty, m, \mathcal{E}}(X) \subseteq \Psi_{c, \Lambda}^{-\infty, m, \mathcal{F}_{1}}(X)$ with $\mathcal{F}_{1, l b}=E_{l b}+1, F_{1, r b}=E_{r b}$, $F_{1, f f}=\mathbb{N} \cup\left(E_{f f}+1\right) \cup\left(E_{l b}+E_{r b}+1\right)$, and $F_{1, f i}=\mathbb{N}$, where we used Lemma 5.1 , and the fact that $x$ vanishes at $l b, f f$, and $f i$ of $\mathcal{T}_{m}$. By Lemma 6.5 , there is a $B_{1}^{\prime} \in \Psi_{c, \Lambda}^{-\infty, m, \mathcal{E}_{1}^{\prime}}(X)$, where $\mathcal{E}_{1}^{\prime}=\left(\widehat{E}^{+}(\alpha) \bar{\cup} F_{1, l b}, \varnothing, F_{1, f f}, \mathbb{N}\right)$, such that

$$
(A-\lambda) x^{m} B_{1}^{\prime}=R_{1} \quad \bmod \Psi_{c, \Lambda}^{-\infty, m,\left(\varnothing, F_{1, r b}, F_{1, f f}, \mathbb{N}\right)}(X) .
$$

Thus, if $B_{2}=B_{1}+B_{1}^{\prime} \in \widetilde{\Psi}_{c, \Lambda}^{-m, m, \mathcal{E}_{2}}(X)$, where $\mathcal{E}_{2}=\mathcal{E}_{1} \cup \mathcal{E}_{1}^{\prime}$, we have,

$$
(A-\lambda) x^{m} B_{2}=\operatorname{Id}-R_{2},
$$

where $R_{2} \in \Psi_{c, \Lambda}^{-\infty, m, \mathcal{F}_{2}}(X)$, where $\mathcal{F}_{2}=\left(\varnothing, F_{1, r b}, F_{1, f f}, \mathbb{N}\right)$. Observe that for each $j, R_{2}^{j} \in \Psi_{c, \Lambda}^{-\infty, m, \mathcal{F}_{2, j}}(X)$, where the $\mathcal{F}_{2, j}$ are defined inductively using Proposition 5.4 and the facts that $R_{2}^{j+1}=R_{2} \circ R_{2}^{j}$ and $\mathcal{F}_{1, f f} \geq 1$ :

$$
\mathcal{F}_{2,1}:=\mathcal{F}_{2}, \mathcal{F}_{2, j+1}:=\left(\varnothing,\left(F_{1, r b}+j\right) \cup F_{2, j, r b}, F_{2, j+1, f f}, \mathbb{N}_{j+1}\right),
$$

where $\mathbb{N}_{j}=\{j, j+1, \ldots\}$ and $F_{2, j, f f}=\left\{\left(z_{1}, k_{1}\right)+\cdots+\left(z_{j}, k_{j}\right) \mid\left(z_{i}, k_{i}\right) \in F_{1, f f}\right\}$. Set $F_{2, r b}^{\prime}:=\bar{\cup}_{r \in \mathbb{N}_{0}}\left(F_{1, r b}+r\right), F_{2, f f}^{\prime}=\cup_{j=1}^{\infty} F_{2, j, f f}$, and $\mathcal{F}_{2}^{\prime}=\left(\varnothing, F_{2, r b}^{\prime}, F_{2, f f}^{\prime}, \mathbb{N}\right)$. Then, $\mathcal{F}_{2, j} \subseteq \mathcal{F}_{2}^{\prime}$ for each $j$ and $\mathcal{F}_{2, j} \rightarrow \mathcal{F}_{2}^{\prime}$ as $j \rightarrow+\infty$ in the sense that $c \in \mathcal{F}_{2}^{\prime}$ if and only if for some $j, c \in \mathcal{F}_{2, j}$. Hence, we can find an asymptotic sum $R_{2}^{\prime} \sim \sum_{j=1}^{\infty} R_{2}^{j} \in \Psi_{c, \Lambda}^{-\infty, m, \mathcal{F}_{2}^{\prime}}(X)$ such that for each $N$,

$$
R_{2}^{\prime}-\sum_{j=1}^{N-1} R_{2}^{j} \in \Psi_{c, \Lambda}^{-\infty, m, \mathcal{F}_{2, N}^{\prime}}(X), \mathcal{F}_{2, N}^{\prime}:=\left(\varnothing, F_{2, r b}^{\prime}, F_{2, N, f f}^{\prime}, \mathbb{N}_{N}\right) .
$$

Set $B_{3}:=B_{2}\left(\mathrm{Id}+R_{2}^{\prime}\right) \in \widetilde{\Psi}_{c, \Lambda}^{-m, m, \mathcal{E}_{3}}(X)$, where (see Theorem 5.7) $\mathcal{E}_{3}=$ $\mathcal{E}_{2} \cup \mathcal{F}_{2}^{\prime} \cup \mathcal{E}_{2} \hat{\circ} \mathcal{F}_{2}^{\prime}$. Then, following through the construction of $B_{3}$, one can 
see that $\mathcal{E}_{3}=\left(E_{3, l b}, E_{3, r b}, E_{3, f f}, \mathbb{N}_{0}\right)$, where $E_{3, l b}>\alpha-m, E_{3, r b}>-(\alpha-m)$, and $E_{3, f f}>0$; and

$$
(A-\lambda) x^{m} B_{3}=\mathrm{Id}-R_{3},
$$

where $R_{3} \in \Psi_{c, \Lambda}^{-\infty, m, \mathcal{F}_{3}}(X)$ with $\mathcal{F}_{3}=\left(\varnothing, F_{2, r b}^{\prime}, \varnothing, \varnothing\right)$. Since $F_{2, r b}^{\prime}>-(\alpha-$ $m$ ), and since $\Psi_{c, \Lambda}^{-\infty, m, \mathcal{F}_{3}}(X) \subseteq \Psi_{\Lambda}^{-\infty, \varnothing, F_{2, r b}^{\prime}}(X)$, by Lemma 6.9 , Id $-R_{3}(\lambda)$ is invertible on $x^{\alpha-m} H_{b}^{s-m}(X)$ for $\lambda \in \Lambda$ sufficiently large, with inverse of the form Id $-R_{3}^{\prime}$, where $R_{3}^{\prime} \in \Psi_{\Lambda}^{-\infty, \varnothing, \mathcal{F}_{2, r b}^{\prime}}(X)$. Hence, $A-\lambda: x^{\alpha} H_{b}^{s}(X) \longrightarrow$ $x^{\alpha-m} H_{b}^{s-m}(X)$ is invertible for all $\lambda \in \Lambda$ large, with inverse,

$$
\begin{gathered}
(A-\lambda)^{-1}=x^{m} B_{3}\left(\operatorname{Id}-R_{3}^{\prime}\right)=x^{m} B_{3}-x^{m} B_{3} R_{3}^{\prime} \in x^{m} \widetilde{\Psi}_{c, \Lambda}^{-m, m, \mathcal{E}_{3}}(X) \\
+x^{m} \Psi_{\Lambda}^{-\infty, E_{3, l b}, F_{2, r b}^{\prime}}(X) \subseteq x^{m} \widetilde{\Psi}_{c, \Lambda}^{-m, m, \mathcal{G}}(X),
\end{gathered}
$$

where $\mathcal{G}=\left(E_{3, l b}, E_{3, r b} \cup F_{2, r b}^{\prime}, E_{3, f f}, \mathbb{N}_{0}\right)$. By Theorem 3.2, for finite $\lambda$, $(A-\lambda)^{-1} \in x^{m} \widetilde{\Psi}_{b}^{-\infty, \mathcal{E}(\alpha)}(X)$. Now, the expansions at $l b, r b$, and $f f$ of $X_{b}^{2}$ must of course, be the same as the expansions at $l b, r b$, and ff of $\mathcal{T}_{m}$ given by $\mathcal{G}$; thus, $(A-\lambda)^{-1} \in x^{m} \widetilde{\Psi}_{c, \Lambda}^{-m, m, \mathcal{E}(\alpha), \mathbb{N}_{0}}(X)$.

\section{The Structure Theorem.}

In this section, we describe the Schwartz kernels of operators in $\Psi_{c, \Lambda}^{m, d}(X)$ as polyhomogeneous functions on certain blown-up manifolds. We begin by describing the polyhomogeneity of the Schwartz kernel when restricted to the diagonal. The blown-up manifold in this case is just $X_{d}$ (see Figure 6 for a picture of $X_{d}$ ) already studied in Section 5.2. This result reduces trace computations to a simple pushforward theorem of Melrose (Lemma 1.1 in the Appendix), see Proposition 8.3. We next describe the whole Schwartz kernel as a polyhomogeneous function, see Theorem 7.2. In this case, we need to introduce a new blown-up manifold tailored to fit the particular homogeneities of our parameter-dependent operators.

Throughout this section, we fix a smooth positive $b$-density on $X$. This trivialization of the $b$-density bundle allows us to omit density factors that are inherent in Schwartz kernels (cf. Definition 2).

Theorem 7.1. Let $A \in \Psi_{c, \Lambda}^{m, d}(X)$ where $m<-n$. Then $\left.K_{A}\right|_{\Delta_{b}}$ is defined, and moreover,

$$
\left.K_{A}\right|_{\Delta_{b}} \in \mathcal{A}_{p h g}^{\mathcal{F}}\left(X_{d}\right)
$$

where $\mathcal{F}$ is the index family on $X_{d}$ defined by

$$
F_{b x}:=\mathbb{N}_{0} ; F_{f i}:=\mathbb{N}_{0} ; F_{b i}:=-m-n+\mathbb{N}_{0} .
$$


In words, $\left.K_{A}\right|_{\Delta_{b}}$ when lifted to $X_{d}$, defines a function which is smooth up to $b x$ and $f i$, and has an expansion at $b i$ with index set $F_{b i}$. The proof of this theorem is straightforward: we just write $\left.K_{A}\right|_{\Delta_{b}}$ in the coordinates on $X_{d}$ and show that it has the desired polyhomogeneity.

Proof. For simplicity, we will assume that $K_{A}$ is supported near $f f\left(X_{b}^{2}\right)$. Let $[0, \infty)_{\varrho} \times \mathbb{R}_{y}^{n-1} \times \mathbb{R}_{z}^{n}$ be a coordinate patch on $X_{b}^{2}$ with $\varrho$ defining $f f\left(X_{b}^{2}\right)$ and with $\Delta_{b} \cong\{z=0\}$. Then by definition of $\Psi_{c, \Lambda}^{m, d}(X)$, for any $N \in \mathbb{N}$ we can express $K_{A}$ as a sum:

$$
K_{A}=\sum_{k=0}^{N-1} A_{m-k}+R_{N}
$$

where

$$
\begin{aligned}
A_{m-k} & :=\int e^{i z \cdot \xi} \chi\left(\varrho^{d} \lambda, \xi\right) a_{m-k}\left(\varrho^{d} \lambda, \varrho, y, \xi\right) d \xi \\
R_{N} & :=\int e^{i z \cdot \xi} r_{N}\left(\varrho^{d} \lambda, \varrho, y, \xi\right) d \xi
\end{aligned}
$$

where $a_{m-k}\left(\delta^{d} \lambda, \varrho, y, \delta \xi\right)=\delta^{m-k} a_{m-k}(\lambda, \varrho, y, \xi)$ for all $\delta>0,(\varrho, y) \mapsto$ $r_{N}(\lambda, \varrho, y, \xi)$ takes values in $S_{\Lambda_{\lambda}}^{m-N, d}\left(\mathbb{R}_{\xi}^{n}\right)$, and where $\chi(\lambda, \xi) \in C^{\infty}\left(\Lambda \times \mathbb{R}^{n}\right)$ with $\chi(\lambda, \xi) \equiv 0$ near $(\lambda, \xi)=0$ and $\chi(\lambda, \xi) \equiv 1$ outside of $(\lambda, \xi)=0$. Note that since $m<-n$, these symbols are integrable in $\xi$ over $\mathbb{R}^{n}$ (see the symbol estimates (4.1)). In particular, $\left.K_{A}\right|_{\Delta_{b}}$ is defined.

We now consider local coordinates on $X_{d}$. Since $[0, \infty)_{\varrho} \times \mathbb{R}_{y}^{n-1} \times \mathbb{R}_{z}^{n}$ is a coordinate patch on $X_{b}^{2}$ with $\varrho$ defining $f f\left(X_{b}^{2}\right)$ and $\Delta_{b} \cong\{z=0\}$, and since $X \cong \Delta_{b}$, we can consider $[0, \infty)_{\varrho} \times \mathbb{R}_{y}^{n-1}$ a coordinate patch on $X$ with $\varrho$ defining $\partial X$. Let $r=|\mu|$ where $\mu=1 / \lambda^{1 / d}$ for $\lambda \in \Lambda$. Then, cf. Figure 6 , near $b x, r$ is a defining function for $f i$ and $v=\varrho / r$ is a defining function for $b x$; and near $b i, \varrho$ is a defining function for $f i$ and $w=r / \varrho$ is a defining function for $b i$.

We now show that $\left.A_{m-k}\right|_{\Delta_{b}} \in \mathcal{A}_{p h g}^{\mathcal{F}}\left(X_{d}\right)$. To do so, we write $\left.A_{m-k}\right|_{\Delta_{b}}$ in the coordinates on $X_{d}$. Consider first the coordinates $r$ and $v=\varrho / r$ near $b x$. Thus, as $\varrho^{d} \lambda=v^{d} \omega^{-d}$ where $\omega=\mu /|\mu|$, we have

$$
\left.A_{m-k}\right|_{\Delta_{b}}=\int \chi\left(v^{d} \omega^{-d}, \xi\right) a_{m-k}\left(v^{d} \omega^{-d}, r v, y, \xi\right) d \xi .
$$

It follows that $\left.A_{m-k}\right|_{\Delta_{b}}$ is smooth in $v$ and $r$.

Now consider the coordinates $\varrho$ and $w=1 / v$ near bi. In this case, $\left.A_{m-k}\right|_{\Delta_{b}}=\int \chi\left(w^{-d} \omega^{-d}, \xi\right) a_{m-k}\left(w^{-d} \omega^{-d}, \varrho, y, \xi\right) d \xi$. Since $\chi(\lambda, \xi) \equiv 1$ outside a neighborhood of $(\lambda, \xi)=0$, for $w$ small, $\chi\left(w^{-d} \omega^{-d}, \xi\right) \equiv 1$. Hence, 
we may just as well replace $\chi$ with 1 . Doing this, and making the change of variables $\xi \mapsto w^{-1} \xi$ then yields

$$
\left.A_{m-k}\right|_{\Delta_{b}}=w^{k-m-n} \int a_{m-k}\left(\omega^{-d}, \varrho, y, \xi\right) d \xi .
$$

It follows that $\left.A_{m-k}\right|_{\Delta_{b}}$ is smooth in $\varrho$ and has an expansion at $w=0$ with index set $k-m-n$.

In summary, we have shown that $\left.A_{m-k}\right|_{\Delta_{b}} \in \mathcal{A}_{\text {phg }}^{\mathcal{F}}\left(X_{d}\right)$, but with $\left.A_{m-k}\right|_{\Delta_{b}}$ actually vanishing to the higher order $-m-n+k$ at $b i$ instead of $-m-n$. By (7.1), we have

$$
\left.K_{A}\right|_{\Delta_{b}}=\left.\sum_{k=0}^{N-1} A_{m-k}\right|_{\Delta_{b}}+\left.R_{N}\right|_{\Delta_{b}} .
$$

A similar analysis as we did with $\left.A_{m-k}\right|_{\Delta_{b}}$ shows that $\left.R_{N}\right|_{\Delta_{b}}$ is smooth up to $b x$ and $f$. Moreover, using the symbol estimates (4.1) for $S_{\Lambda}^{m-N, d}\left(\mathbb{R}^{n}\right)$, it is straightforward to verify that $\left.R_{N}\right|_{\Delta_{b}}$ vanishes to higher and higher order at $b i$ as $N$ is chosen larger and larger. It follows that $\left.K_{A}\right|_{\Delta_{b}} \in \mathcal{A}_{\text {phg }}^{\mathcal{F}}\left(X_{d}\right)$.

We now describe the whole Schwartz kernel $K_{A}$ of an operator $A \in$ $\Psi_{c, \Lambda}^{m, d}(X)$ as a polyhomogeneous function on a blown-up manifold. We begin by defining the blown-up manifold:

$$
X_{d}^{2}:=\left[\mathcal{T}_{d} ; \partial_{\infty} \bar{\Lambda}_{d} \times \Delta_{b} ; \bar{\Lambda}_{d} \times \Delta_{b}\right] .
$$

Here, $\mathcal{T}_{d}$ is defined in (5.1), and recall that (see Section 5.1) $\Lambda_{d}:=\Lambda^{1 / d}$ and that $\bar{\Lambda}_{d}$ denotes the radial compactification of $\Lambda_{d}$ with $\partial_{\infty} \bar{\Lambda}_{d}$ denoting the boundary at " $\lambda^{1 / d}=\infty$ ". Figure 7 shows how $X_{d}^{2}$ is constructed. Thus, $X_{d}^{2}$ is constructed as follows: First, in $\bar{\Lambda}_{d} \times X_{b}^{2}$, we blow-up $\left\{\lambda^{1 / d}=\infty\right\} \times f f$ to form $\mathcal{T}_{d}$. If $\Delta_{\infty}$ (the "diagonal at $\lambda^{1 / d}=\infty$ ") is the lift of $\partial_{\infty} \bar{\Lambda}_{d} \times \Delta_{b}$ into $\mathcal{T}_{d}$, then blowing it up in $\mathcal{T}_{d}$, we form $\left[\mathcal{T}_{d} ; \Delta_{\infty}\right]$. The lift of $\bar{\Lambda}_{d} \times \Delta_{b}$ in this new blown-up space is denoted by $\Delta_{f}$. Finally, blowing-up $\Delta_{f}$ in $\left[\mathcal{T}_{d} ; \Delta_{\infty}\right]$ creates $X_{d}^{2}$.

If $\beta: X_{d}^{2} \longrightarrow \bar{\Lambda}_{d} \times X_{b}^{2}$ is the blow-down map, then the new faces from $\mathcal{T}_{d}$ that we create in $X_{d}^{2}$ are $d i:=\beta^{*}\left(\partial_{\infty} \bar{\Lambda}_{d} \times \Delta_{b}\right)$, diagonal at infinity; and $d f:=\beta^{*}\left(\bar{\Lambda}_{d} \times \Delta_{b}\right)$, diagonal face.

Theorem 7.2. The Schwartz kernel of an operator $A \in \Psi_{c, \Lambda}^{m, d}(X)$ lifts into $X_{d}^{2}$ to define a polyhomogeneous function:

$$
K_{A} \in \mathcal{A}_{\text {phg }}^{\mathcal{E}}\left(X_{d}^{2}\right)
$$




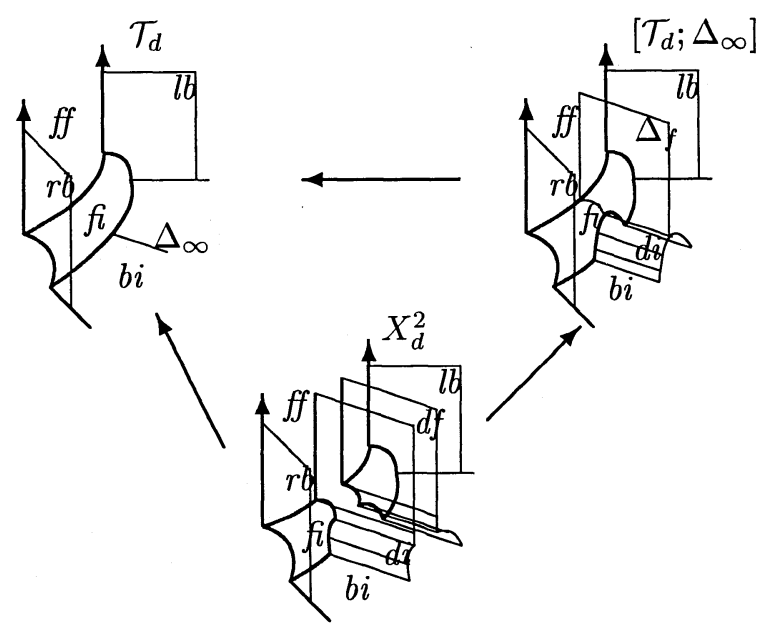

Figure 7: The manifold $X_{d}^{2}$. The arrows represent blow-down maps.

where $\mathcal{E}$ is the index family on $X_{d}^{2}$ defined by

$$
\begin{gathered}
E_{f f}=E_{f i}:=\mathbb{N}_{0} ; E_{l b}=E_{r b}=E_{b i}:=\varnothing ; \\
E_{d f}:=\left(-m-n+\mathbb{N}_{0}\right) \cup \mathbb{N}_{0} ; E_{d i}:=-m-n+\mathbb{N}_{0} .
\end{gathered}
$$

In words, $K_{A}$ when lifted to $X_{d}^{2}$ defines a function which is smooth up to $f f$ and $f i$, vanishes to infinite order at $l b, r b$, and $b i$, and has expansions at the diagonal face $d f$ and the diagonal at infinity $d i$, with index sets $E_{d f}$ and $E_{d i}$ respectively. The proof of Theorem 7.2 is simple in principle: we write $K_{A}$ in local coordinates on $X_{d}^{2}$ and prove that it has the claimed asymptotic properties. However, as its proof is a bit technical, the proof may be skipped without loss of continuity.

Proof. Our first order of business is to describe the local coordinates on $X_{d}^{2}$. As usual, let $\mu=1 / \lambda^{1 / d}$ for $\lambda \in \Lambda, r:=|\mu|$, and $\omega:=\mu /|\mu| \in \mathbb{S}_{d}^{1}$, where $\mathbb{S}_{d}^{1}=\left\{\omega=\mu /|\mu|: \mu=1 / \lambda^{1 / d}\right.$ for $\left.\lambda \in \Lambda\right\}$. Let $[0, \infty)_{\varrho} \times \mathbb{R}_{y}^{n-1} \times \mathbb{R}_{z}^{n}$ be a coordinate patch on $X_{b}^{2}$ with $\varrho$ defining $f f\left(X_{b}^{2}\right)$ and $\Delta_{b} \cong\{z=0\}$. Then near $f f\left(X_{d}^{2}\right)$, we have

$$
X_{d}^{2} \cong \mathcal{V}_{d}, \quad \text { with } \mathcal{V}_{d}:=[0, \infty)_{r} \times \mathbb{S}_{d}^{1} \times[0, \infty)_{v} \times\left[\mathbb{R}_{z}^{n} ;\{0\}\right] \times \mathbb{R}_{y}^{n-1},
$$

where $r$ and $v=\varrho / r$ define $f i$ and $f f$ respectively. Away from $f f\left(X_{d}^{2}\right)$,

$$
X_{d}^{2} \cong \mathcal{W}_{d}, \quad \text { with } \mathcal{W}_{d}:=[0, \infty)_{\varrho} \times \mathbb{S}_{d}^{1} \times \Theta \times \mathbb{R}_{y}^{n-1},
$$


where if $w=r / \varrho$, then

$$
\Theta:=\left[[0, \infty)_{w} \times \mathbb{R}_{z}^{n} ;[0, \infty)_{w} \times\{z=0\} ;\{w=0\} \times\{z=0\}\right],
$$

Note that $\varrho$ and $w$ define $f i$ and $b i$ respectively, and that $d f$ and $d i$ are those faces in $\mathcal{V}_{d}$ created from the blow-ups of $[0, \infty)_{w} \times\{z=0\}$ and $\{w=$ $0\} \times\{z=0\}$ respectively.

Before analyzing the asymptotics of $K_{A}$, we remark that if $\varrho^{d} \lambda$ is replaced with $\lambda$, then the asymptotics of $K_{A}$ are analyzed in [29]. Thus, in the arguments that follow, we will rely heavily on the results of [29]. Now write $K_{A}$ as in (7.1) in the proof of Theorem 7.1. We will now analyze each term of (7.1) separately.

We first show that $A_{m-k} \in \mathcal{A}^{\mathcal{E}}\left(X_{d}^{2}\right)$. We begin in the coordinates of $\mathcal{W}_{d}$. Indeed, if $w=r / \varrho$, then as $\varrho^{d} \lambda=\varrho^{d} r^{-d} \omega^{-d}=w^{-d} \omega^{-d}$, we can write

$$
A_{m-k}=\int e^{i z \cdot \xi} \chi\left(w^{-d} \omega^{-d}, \xi\right) a_{m-k}\left(w^{-d} \omega^{-d}, \varrho, y, \xi\right) d \xi .
$$

Now $A_{m-k}$ is an operator of the form considered in [29]. Then Lemmas 4.39, 4.41, and 4.43 of [29] show that $A_{m-k} \in \mathcal{A}^{\mathcal{E}}\left(\mathcal{W}_{d}\right)$.

Consider now the coordinates $\mathcal{V}_{d}$. Thus, observe that since $\varrho^{d} \lambda=$ $\varrho^{d} r^{-d} \omega^{-d}=v^{d} \omega^{-d}$ where $v=\varrho / r$, we can write

$$
A_{m-k}=\int e^{i z \cdot \xi} \chi\left(v^{d} \omega^{-d}, \xi\right) a_{m-k}\left(v^{d} \omega^{-d}, \xi\right) d \xi .
$$

Also observe that outside a neighborhood of $\xi=0, \chi\left(v^{d} \omega^{-d}, \xi\right)-\chi(0, \xi) \equiv$ 0 . Thus, $\int e^{i z \cdot \xi}\left(\chi\left(v^{d} \omega^{-d}, \xi\right)-\chi(0, \xi)\right) a_{m-k}\left(v^{d} \omega^{-d}, \xi\right) d \xi$ is smooth in the variables $z$ and $v^{d}$. Hence, it suffices to show that

$$
\int e^{i z \cdot \xi} \chi(\xi) a_{m-k}\left(v^{d} \omega^{-d}, \xi\right) d \xi \in \mathcal{A}^{\mathcal{E}}\left(\mathcal{V}_{d}\right)
$$

where $\chi(\xi):=\chi(0, \xi)$. The proof of this statement follows from the proof of [29, Lem. 4.40]. It follows that $A_{m-k} \in \mathcal{A}^{\mathcal{E}}\left(X_{d}^{2}\right)$.

We now focus on the remainder term $R_{N}$. Suppose that $m-N \leq$ $-2 M-n-1$ where $M \in \mathbb{N}$. Then, we claim that $R_{N} \in \sigma^{M} S^{0}\left(\mathcal{W}_{d}\right)$, where $\sigma$ is a total boundary defining function for the hypersurfaces $d f, d i$, and $b i$, of $\mathcal{W}_{d}$. Also, we claim that $R_{N} \in C^{2 M}\left(\mathcal{V}_{d}\right)$.

Indeed, if $w=r / \varrho$, then $R_{N}=\int e^{i z \cdot \xi} r_{N}\left(w^{-d} \omega^{-d}, \varrho, y, \xi\right) d \xi$. We can now apply [29, Lem. 4.45] to prove that $R_{N} \in \sigma^{M} S^{0}\left(\mathcal{W}_{d}\right)$.

To see that $R_{N} \in C^{2 M}\left(\mathcal{V}_{d}\right)$, we first recall the following fact: let $a(\xi) \in$ $S^{p}\left(\mathbb{R}^{n}\right)$ with $p<-n-\ell$, where $\ell \in \mathbb{N}_{0}$, and let $u=\int e^{i z \cdot \xi} a(\xi) d \xi$. Then, $u \in C^{\ell}\left(\mathbb{R}^{n}\right) \cap L^{\infty}\left(\mathbb{R}^{n}\right)$. 
Now, $R_{N}=\int e^{i z \cdot \xi} r_{N}\left(v^{d} \omega^{-d}, \varrho, y, \xi\right) d \xi$, where $\varrho^{d} \lambda=v^{d} \omega^{-d}$. From the estimates (4.1), $S_{\Lambda}^{m-N, d}\left(\mathbb{R}^{n}\right) \subseteq C^{\infty}\left(\Lambda ; S^{-n-M-1}\left(\mathbb{R}^{n}\right)\right)$. Thus, the fact mentioned in the previous paragraph implies that $R_{N} \in C^{2 M}\left(\mathcal{V}_{d}\right)$.

The fact that $A_{m-k} \in \mathcal{A}^{\mathcal{E}}\left(X_{d}^{2}\right)$ for each $k$, and the above analysis of $R_{N}$ together with the fact that $N \in \mathbb{N}$ can be made arbitrarily large, complete the proof of Theorem 7.2.

\section{Applications.}

Throughout this section, $A \in x^{-m} \operatorname{Diff}_{b}^{m}(X)$ will be fully elliptic with respect to $\alpha \in \mathbb{R}$ on a closed sector $\Lambda$. In Theorems 8.1 and 8.2, we realize the Schwartz kernel of $(A-\lambda)^{-1}$ as polyhomogeneous distributions on blown-up manifolds. In Theorem 8.4 we prove a trace expansion for the resolvent. Finally, in Section 8.3 we investigate the heat trace expansion.

\subsection{The resolvent kernel.}

By Theorem 6.1, for $\lambda \in \Lambda$ sufficiently large, we have

$$
(A-\lambda)^{-1} \in x^{m} \widetilde{\Psi}_{c, \Lambda}^{-m, m, \mathcal{E}(\alpha), \mathbb{N}_{0}}(X) \subseteq x^{m} \Psi_{c, \Lambda}^{-m, m}(X)+\widehat{\Psi}_{c, \Lambda}^{-\infty, m, \mathcal{E}_{1}(\alpha)}(X),
$$

where the "बै space" is defined in (5.8), and where $\mathcal{E}_{1}(\alpha)=\left(\check{E}^{+}(\alpha)+\right.$ $\left.m, \check{E}^{-}(\alpha), E(\alpha)+m, \mathbb{N}_{m}\right)$ with $\mathbb{N}_{m}=\{m, m+1, \ldots\}$. Thus, for any $N \in \mathbb{N}$ and $\lambda$ sufficiently large,

$$
(A-\lambda)^{-N} \in x^{N m} \Psi_{c, \Lambda}^{-N m, m}(X)+\widehat{\Psi}_{c, \Lambda}^{-\infty, m, \mathcal{E}_{N}(\alpha)}(X),
$$

where the index family $\mathcal{E}_{N}(\alpha)=\left(E_{N, l b}(\alpha), E_{N, r b}(\alpha), E_{N, f f}(\alpha), \mathbb{N}_{m N}\right)$ is a combination of, and defined inductively from, the index sets of $\mathcal{E}_{1}(\alpha)$ using Proposition 5.6. In Theorems 8.1 and 8.2 below, we fix a smooth positive $b$-density on $X$ so that we can omit density factors in Schwartz kernels. Our first result involves the manifold $X_{d}$ in Figure 6 with $d=m$.

Theorem 8.1. Let $B \in \operatorname{Diff}_{b}^{m^{\prime}}(X), m^{\prime} \in \mathbb{N}_{0}$, and let $A \in x^{-m} \operatorname{Diff}_{b}^{m}(X)$ be fully elliptic with respect to $\alpha \in \mathbb{R}$ on $\Lambda$. If $m^{\prime}-m N<-n$, then for $\lambda \in \Lambda$ sufficiently large, identifying $B(A-\lambda)^{-N}$ with its kernel, we have

$$
\left.B(A-\lambda)^{-N}\right|_{\Delta_{b}} \in \mathcal{A}_{\text {phg }}^{\mathcal{G}}\left(X_{m}\right),
$$

where $\mathcal{G}=\left(G_{b x}, G_{f i}, G_{b i}\right)$ is an index family on $X_{m}$ such that

$$
G_{b x} \geq m ; G_{f i}:=\mathbb{N}_{m N} ; G_{b i}:=-m^{\prime}+m N-n+\mathbb{N}_{0} .
$$


The exact form of $G_{b x}$ is not important; this is why we don't explicitly state it (it is however, given in the proof).

Proof. As $\operatorname{Diff}_{b}^{m^{\prime}}(X) \subseteq \Psi_{c, \Lambda}^{m^{\prime}, m}(X)$ (this is easily verified), the composition results of the full calculus (see Section 5.3) imply that

$$
B(A-\lambda)^{-N}=C+D,
$$

where $C \in x^{N m} \Psi_{c, \Lambda}^{m^{\prime}-N m, m}(X)$ and $D \in \widehat{\Psi}_{c, \Lambda}^{-\infty, m, \mathcal{E}_{N}(\alpha)}(X)$. By Theorem $7.1,\left.K_{C}\right|_{\Delta_{b}}$ is a polyhomogeneous function on $X_{m}$ with expansions given by an index family satisfying (8.1). (In this case, $\left.K_{C}\right|_{\Delta_{b}}$ has an expansion at $b x$ with index set $\mathbb{N}_{m N}$.) Since operators in $\widehat{\Psi}_{c, \Lambda}^{-\infty, m, \mathcal{E}_{N}(\alpha)}(X)$ are defined directly in terms of the polyhomogeneity of their kernels, see Definition 3, the definition (5.8), and the second statement in Lemma 5.1, it immediately follows that $\left.K_{D}\right|_{\Delta_{b}}$ is a polyhomogeneous function on $X_{m}$ with expansions given by an index family satisfying (8.1). (In this case, $\left.K_{D}\right|_{\Delta_{b}}$ vanishes to infinite order at $b i$ and has an expansion at $b x$ with index set $G=$ $\mathbb{N}_{m N} \cup E_{N, f f}(\alpha) \cup\left(E_{N, l b}(\alpha)+E_{N, r b}(\alpha)\right)$. One can check that $G \geq m$.)

We now consider the asymptotics of the whole Schwartz kernel. To understand exactly what the following result means, we refer the reader to Section 7 and Section 5.1 for the definitions of the manifolds $X_{m}^{2}$ and $\mathcal{T}_{m}$ respectively. Each of these manifolds is designed to capture the singularities of the resolvent at $\lambda=\infty$, at $\Delta_{b}$, and at $\partial X$.

Theorem 8.2. Let $B \in \operatorname{Diff}_{b}^{m^{\prime}}(X), m^{\prime} \in \mathbb{N}_{0}$, let $A \in x^{-m} \operatorname{Diff}_{b}^{m}(X)$ be fully elliptic with respect to $\alpha \in \mathbb{R}$ on $\Lambda$, and let $N \in \mathbb{N}$. Then for $\lambda \in \Lambda$ sufficiently large, identifying $B(A-\lambda)^{-N}$ with its kernel, we have

$$
B(A-\lambda)^{-N} \in \mathcal{A}_{p h g}^{\mathcal{E}}\left(X_{m}^{2}\right)+\mathcal{A}_{p h g}^{\mathcal{F}}\left(\mathcal{T}_{m}\right)+\mathcal{A}_{p h g}^{\mathcal{G}}\left(\bar{\Lambda} \times X^{2}\right),
$$

where $\mathcal{F}=\left(\mathcal{E}_{N}(\alpha), \varnothing\right)$ with $\varnothing$ associated to bi, $\mathcal{G}=\left(\varnothing, E_{N, l b}(\alpha), E_{N, r b}(\alpha)\right)$ with $\varnothing$ associated to $\partial_{\infty} \bar{\Lambda}$, and where

$$
\begin{aligned}
E_{l b}=E_{r b}=E_{b i}:=\varnothing ; & E_{f f}=E_{f i}:=\mathbb{N}_{m N} ; \\
E_{d f}:=\left(-m^{\prime}+m N-n+\mathbb{N}_{0}\right) \cup \mathbb{N}_{0} ; & E_{d i}:=\left(-m^{\prime}+m N-n+\mathbb{N}_{0}\right) .
\end{aligned}
$$

Proof. We can decompose $B(A-\lambda)^{-N}=C+D$ as in (8.2) of Theorem 8.1. Then Theorem 7.2 implies that $K_{C} \in \mathcal{A}_{p h g}^{\mathcal{E}}\left(X_{m}^{2}\right)$. Since operators 
in $\widehat{\Psi}_{c, \Lambda}^{-\infty, m, \mathcal{E}_{N}(\alpha)}(X)$ are defined directly in terms of the polyhomogeneity of their kernels, see Definition 3, the definition (5.8), and the definition (5.3), it follows automatically that $K_{D} \in \mathcal{A}_{p h g}^{\mathcal{F}}\left(\mathcal{T}_{m}\right)+\mathcal{A}_{p h g}^{\mathcal{G}}\left(\bar{\Lambda} \times X^{2}\right)$.

\subsection{Trace expansion of the resolvent.}

Recall that $X_{d}$ is $\bar{\Lambda}_{d} \times X$ blown-up at $\left\{\lambda^{1 / d}=\infty\right\} \times Y$, where $\bar{\Lambda}_{d}$ is $\Lambda_{d}$ $\left(=\Lambda^{1 / d}\right)$ radially compactified, see (5.5) and Figure 6. Also recall that $r=|\mu|$ and $\omega=\mu /|\mu|$, where $\mu=1 / \lambda^{1 / d}$ with $\lambda \in \Lambda$, are coordinates on $\bar{\Lambda}_{d}$, with $r$ defining $\left\{\lambda^{1 / d}=\infty\right\}$. Let $u \in \mathcal{A}_{\text {phg }}^{\mathcal{E}}\left(X_{d}\right)$. Then for fixed $r>0$, we can consider $u(r, \omega) \in \mathcal{A}_{p h g}^{E_{b x}}(X)$. In particular, given $0<\nu \in C^{\infty}\left(X, \Omega_{b}\right)$, if $E_{b x}>0$, then $u(r, \omega) \nu$ is integrable over $X$. The following result, which is really just an application of Melrose's pushforward theorem (see Lemma 1.1 in the Appendix), states how $\int_{X} u(r, \omega) \nu$ behaves as $r \downarrow 0$.

Proposition 8.3. Let $u \in \mathcal{A}_{\text {phg }}^{\mathcal{E}}\left(X_{d}\right)$ with $E_{b x}>0$. Then, as $r \downarrow 0$, $\int_{X} u(r, \omega) \nu$ has an asymptotic expansion

$$
\int_{X} u(r, \omega) \nu \sim \sum_{(z, k) \in E_{f i} \bar{\Xi} E_{b i}} r^{z}(\log r)^{k} u_{(z, k)}(\omega),
$$

where $u_{(z, k)}(\omega)$ are smooth functions of $\omega$.

Proof. We can write $u$ as a sum of three functions $u=u_{1}+u_{2}+u_{3}$, where $u_{1}$ is supported near $b x$ and away from $b i, u_{2}$ is supported near $f i b i$ and away from $b x$, and where $u_{3}$ is supported away from both $b x$ and $f$. (See Figure 6 for a pictorial representation of $X_{d}$.) Then,

$$
\int_{X} u(r, \omega) \nu=\int_{X} u_{1}(r, \omega) \nu+\int_{X} u_{2}(r, \omega) \nu+\int_{X} u_{3}(r, \omega) \nu .
$$

Since for any $(r, \omega), u_{3}(r, \omega)$ is uniformly supported in the interior of $X$, it follows that $\int_{X} u_{3}(r, \omega) \nu$ has an expansion as in (8.3) but with only the index set $E_{b i}$.

Now we work on $\int_{X} u_{1}(r, \omega) \nu$. Decompose $X \cong[0,1)_{x} \times Y$ near $Y=\partial X$. Then near $b x$ and away from $b i, X_{d} \cong[0, \infty)_{r} \times[0, \infty)_{v} \times Y$, where $v=x / r$. Let $u_{1} \nu=v_{1}(r, \omega, v, y)\left|\frac{d x}{x} d y\right|$, where $v_{1}(r, \omega, v, y)$ has an expansion at $r=0$ and $v=0$ with index sets $E_{f i}$ and $E_{b x}$ respectively. Thus, $\int_{X} u_{1}(r, \omega) \nu=\int v_{1}(r, \omega, x / r, y) \frac{d x}{x} d y$. Changing coordinates $x \mapsto r x$, we 
find that $\int_{X} u_{1}(r, \omega) \nu=\int v_{1}(r, \omega, x, y) \frac{d x}{x} d y$. It follows that $\int_{X} u_{1}(r, \omega) \nu$ has an expansion as in (8.3) but with only the index set $E_{f i}$.

Finally, we work on $\int_{X} u_{2}(r, \omega) \nu$. Near $f i \cap b i$ and away from $b x$, we can decompose $X_{d} \cong[0,1)_{x} \times[0, \infty)_{w} \times Y$, where $w=r / x$. Let $u_{2} \nu=v_{2}(x, w, \omega, y)\left|\frac{d x}{x} d y\right|$, where $v_{2}(x, w, \omega, y)$ has an expansion at $x=0$ and $w=0$ with index sets $E_{f i}$ and $E_{b i}$ respectively. Then, $\int_{X} u_{2}(r, \omega) \nu=$ $\int v_{2}(x, r / x, \omega, y) \frac{d x}{x} d y$. Hence, by Lemma 1.1 in the Appendix, it follows that $\int_{X} u_{2}(r, \omega) \nu$ has an expansion as in (8.3).

Theorem 8.4. Let $A \in x^{-m} \operatorname{Diff}_{b}^{m}(X)$ be fully elliptic with respect to $\alpha \in$ $\mathbb{R}$ on $\Lambda$ and let $B \in x^{-\beta} \operatorname{Diff}_{b}^{m^{\prime}}(X), \beta \in \mathbb{R}$ and $m^{\prime} \in \mathbb{N}_{0}$. Suppose that $m^{\prime}-m N<-n$ and that $\beta<m$. Then $B(A-\lambda)^{-N}$ is trace class on $x^{\alpha-m} L_{b}^{2}(X)$ and as $\lambda \rightarrow \infty$ in $\Lambda$, we have

$$
\operatorname{Tr} B(A-\lambda)^{-N} \sim \sum_{k=0}^{\infty} a_{k} \lambda^{\frac{m^{\prime}+n-k}{m}-N}+\sum_{k=0}^{\infty}\left\{b_{k} \log \lambda+c_{k}\right\} \lambda^{\frac{\beta-k}{m}-N},
$$

where $b_{k}=0$ unless $k \in \beta-m^{\prime}-n+\mathbb{N}_{0}$. In particular, as $\lambda \rightarrow \infty$ in $\Lambda$,

$$
\operatorname{Tr}(A-\lambda)^{-N} \sim \sum_{k=0}^{\infty} \lambda^{\frac{n-k}{m}-N} \alpha_{k}+\sum_{k=0}^{\infty} \lambda^{-k / m-N} \log \lambda \beta_{k} .
$$

Proof. First of all, decomposing $B(A-\lambda)^{-N}$ as in (8.2) of Theorem 8.1 (now with an extra factor of $x^{-\beta}$ ), and using standard facts about the $b$-calculus (see for instance, [35, Ch. 4.18]), it follows that $B(A-\lambda)^{-N}$ is trace class on $x^{\alpha-m} L_{b}^{2}(X)$ with $\operatorname{Tr} B(A-\lambda)^{-N}=\left.\int_{X}\left\{B(A-\lambda)^{-N}\right\}\right|_{\Delta_{b}}$. Now, by Theorem $8.2,\left.B(A-\lambda)^{-N}\right|_{\Delta_{b}}$ is of the form $u \nu$, where $0<\nu \in C^{\infty}\left(X, \Omega_{b}\right)$, and where $u \in \mathcal{A}_{\text {phg }}^{\mathcal{G}}\left(X_{m}\right)$, where $\mathcal{G}$ is an index family satisfying

$$
G_{b x} \geq-\beta+m ; G_{f i}:=-\beta+\mathbb{N}_{m N} ; G_{b i}:=-m^{\prime}+m N-n+\mathbb{N}_{0} .
$$

Since $G_{b x}>0$, and since

$$
\begin{aligned}
G_{f i} \bar{\cup} G_{b i}=\left(-m^{\prime}-n+m N+\mathbb{N}_{0}\right) \cup\left(-\beta+m N+\mathbb{N}_{0}\right) \cup \\
\left\{(k-\beta+m N, 1) \mid k \in \mathbb{N}_{0}, k \in \beta-m^{\prime}-n+\mathbb{N}_{0}\right\},
\end{aligned}
$$

Proposition 8.3 implies the trace expansion (8.4), but with $\lambda^{1 / m}$ replaced with $r^{-1}$ and with the coefficients functions of $\omega$. Since $B(A-\lambda)^{-N}$ is holomorphic in $\lambda$, [29, Prop. 5.49] implies that the expansion holds as given in (8.4). 


\subsection{Trace expansion of the heat kernel.}

In this section, we will assume that $\Lambda$ is of the form $\left\{\lambda \in \mathbb{C} \mid \varepsilon_{0} \leq \arg (\lambda) \leq\right.$ $\left.2 \pi-\varepsilon_{0}\right\}$ for some $0<\varepsilon_{0}<\pi / 2$. The heat operator of $A, e^{-t A}$, is defined by the Cauchy integral

$$
e^{-t A}:=\frac{i}{2 \pi} \int_{\Gamma} e^{-t \lambda}(A-\lambda)^{-1} d \lambda,
$$

where $\Gamma$ is an anti-clockwise contour in $\Lambda$ of the form

$$
\Gamma=a+\{\lambda \in \mathbb{C} \mid \arg (\lambda)=\delta \text { or } \arg (\lambda)=2 \pi-\delta\}, \quad a<0, \varepsilon_{0}<\delta<\pi / 2 .
$$

Since $e^{-t \lambda} \rightarrow 0$ exponentially as $\lambda \rightarrow \infty$ on $\Gamma$, the definition of the full calculus (5.4) implies that the integral (8.5) converges uniformly in $x^{m} \widetilde{\Psi}_{b}^{-m, \mathcal{E}(\alpha)}(X)$ for $t>0$.

Integrating by parts $N-1$ times, we can rewrite (8.5) as

$$
e^{-t A}=\frac{i}{2 \pi} \frac{(-t)^{-N+1}}{(N-1) !} \int_{\Gamma} e^{-t \lambda}(A-\lambda)^{-N} d \lambda ;
$$

and hence, $e^{-t A}$ is actually of order $-m N$ rather than $N$. Thus, in fact, $e^{-t A}$ is of order $-\infty$. Hence, if $B$ is any $b$-differential operator, the kernel of $B e^{-t A}$ restricted to $\Delta_{b}$ is defined. The proof of the following corollary follows from the results of [3, Sec. 4.6] applied to $B$ times the Laplace transform (8.6), together with the asymptotic expansion (8.4).

Corollary 8.5. Let $A \in x^{-m} \operatorname{Diff}_{b}^{m}(X)$ be fully elliptic with respect to $\alpha \in \mathbb{R}$ on $\Lambda$ and let $B \in x^{-\beta} \operatorname{Diff}_{b}^{m^{\prime}}(X)$ where $\beta<m$ and $m^{\prime} \in \mathbb{N}_{0}$. Then, as $t \downarrow 0$,

$$
\operatorname{Tr} B e^{-t A} \sim \sum_{k=0}^{\infty} \alpha_{k} t^{\frac{k-m^{\prime}-n}{m}}+\sum_{k=0}^{\infty}\left\{\beta_{k} \log t+\gamma_{k}\right\} t^{\frac{k-\beta}{m}},
$$

where $\beta_{k}=0$ unless $k \in \beta-m^{\prime}-n+\mathbb{N}_{0}$. In particular, as $t \downarrow 0$,

$$
\operatorname{Tr} e^{-t A} \sim \sum_{k=0}^{\infty} t^{\frac{k-n}{m}} \zeta_{k}+\sum_{k=0}^{\infty} t^{k / m} \log t \zeta_{k}^{\prime}
$$

\section{Appendix: Pushforwards and the $b$-calculus.}

The following lemma is the basic version of Melrose's pushforward theorem, see [34]. We will use the notation of Section 2.1. 
Lemma 1.1. Let $f:[0,1)^{2} \longrightarrow[0,1)$ be the map $f(x, y)=x y$. Then given any compactly supported b-density $u=u(x, y)\left|\frac{d x}{x} \frac{d y}{y}\right|$, where $u(x, y)$ has an expansion at $x=0$ with some index set $E_{l b}$ and an expansion at $y=0$ with some index set $E_{r b}$, then

$$
f_{*} u(x)=\int_{0}^{1} u(y, x / y) \frac{d y}{y}=\int_{0}^{1} u(x / y, y) \frac{d y}{y} \in \mathcal{A}_{p h g}^{E_{l l} \bar{\cup} E_{r b}}([0,1)),
$$

where $E_{l b} \bar{\cup} E_{r b}:=E_{l b} \cup E_{r b} \cup\left\{(z, k+\ell+1) \mid(z, k) \in E_{l b},(z, \ell) \in E_{r b}\right\}$.

Proof. Let $\varphi \in C_{c}^{\infty}((0,1))$. Then,

$$
\begin{aligned}
<f_{*} u, \varphi>=<u, f^{*} \varphi> & =\int_{0}^{1} \int_{0}^{1} u(x, y) \varphi(x y) \frac{d x}{x} \frac{d y}{y} \\
& =\int_{0}^{1} \int_{0}^{1} u(x / y, y) \varphi(x) \frac{d x}{x} \frac{d y}{y}(x \mapsto x / y) \\
& =\int_{0}^{1}\left\{\int_{0}^{1} u(x / y, y) \frac{d y}{y}\right\} \varphi(x) \frac{d x}{x} .
\end{aligned}
$$

Thus, $f_{*} u(x)=\int_{0}^{1} u(x / y, y) \frac{d y}{y}$. Changing variables $y \mapsto x / y$ gives the other representation $f_{*} u(x)=\int_{0}^{1} u(y, x / y) \frac{d y}{y}$. That $f_{*} u$ has an expansion at $x=0$ with index set $E_{l b} \cup E_{r b}$ can be found in, for instance, [34].

We now show how to express the composition of $b$-pseudodifferential operators, and their action on functions, in terms of the pushforward of a $b$-density involving their Schwartz kernels.

We will use the notation of Section 3.1. First we start with the mapping properties. Let $A \in \Psi_{b}^{m}(X)$. Let $K$ denote the Schwartz kernel of $A$ as a distribution on $X^{2}$, and let $K_{A}:=\beta^{*} K$ be Schwartz kernel on $X_{b}^{2}$, as given in Definition 1. Here, $\beta: X_{b}^{2} \longrightarrow X^{2}$ is the blow-down map. Let $\varphi \in C^{\infty}(X)$. We will write $A \varphi$ as a pushforward of a $b$-density. Thus, let $\mu \in C^{\infty}\left(X, \Omega_{b}\right)$ be any $b$-density, and let $\pi_{L}, \pi_{R}: X^{2} \longrightarrow X$ be the projections onto the left and right factors of $X^{2}$ respectively. Then a definition chase shows that as distributions,

$$
\mu A \varphi \equiv\left(\pi_{L}\right)_{*}\left(\pi_{R}^{*} \varphi \pi_{L}^{*} \mu K\right)
$$


We will now write this formula in terms of $K_{A}$. To do so, we first define $\pi_{L, b}:=\pi_{L} \circ \beta$ and $\pi_{R, b}:=\pi_{R} \circ \beta$. Then (1.2) takes the form

$$
\begin{aligned}
\mu A \varphi=\left(\pi_{L}\right)_{*}\left(\pi_{R}^{*} \varphi \pi_{L}^{*} \mu K\right) & =\left(\pi_{L}\right)_{*}\left(\beta_{*}\left(\beta^{*}\left(\pi_{R}^{*} \varphi \pi_{L}^{*} \mu\right) K_{A}\right)\right) \\
& =\left(\pi_{L} \circ \beta\right)_{*}\left(\left(\pi_{R} \circ \beta\right)^{*} \varphi\left(\pi_{L} \circ \beta\right)^{*} \mu K_{A}\right) \\
& =\left(\pi_{L, b}\right)_{*}\left(\pi_{R, b}^{*} \varphi \pi_{L, b}^{*} \mu K_{A}\right) .
\end{aligned}
$$

Thus,

$$
\mu A \varphi=\left(\pi_{L, b}\right)_{*}\left(\pi_{R, b}^{*} \varphi \pi_{L, b}^{*} \mu K_{A}\right)
$$

Thus,

$$
A: C^{\infty}(X) \longrightarrow C^{\infty}(X) \Longleftrightarrow\left(\pi_{L, b}\right)_{*}\left(\pi_{R, b}^{*} \varphi \pi_{L, b}^{*} \mu K_{A}\right) \in C^{\infty}\left(X, \Omega_{b}\right) .
$$

A similar argument shows that if $\dot{C}^{\infty}(X)$ is the space of smooth functions vanishing to infinite order at $Y=\partial X$, then

$$
A: \dot{C}^{\infty}(X) \longrightarrow \dot{C}^{\infty}(X) \Longleftrightarrow\left(\pi_{L, b}\right)_{*}\left(\pi_{R, b}^{*} \varphi \pi_{L, b}^{*} \mu K_{A}\right) \in \dot{C}^{\infty}\left(X, \Omega_{b}\right)
$$

for all $\varphi \in \dot{C}^{\infty}(X)$. The proofs of (1.4) and (1.5) use Lemma 1.1, and are very similar to (but much simpler than) the proof of statement $(C)$ in Lemma 4.2. Note that the role of the auxiliary $b$-density $\mu$ is only to ensure that we are pushing forward $b$-densities in (1.4) and (1.5).

We now consider the composition of two $b$-pseudodifferential operators $A_{1}$ and $A_{2}$. To do so, we will write the composition using pullbacks and pushforwards. But first, we must define the $b$-triple product. Define $\pi_{F}, \pi_{S}, \pi_{C}: X^{3} \longrightarrow X^{2}$ by

$$
\pi_{F}(x, y, z)=(x, y), \pi_{S}(x, y, z)=(y, z), \pi_{C}(x, y, z)=(x, z),
$$

and define $\mathcal{T}:=Y \times Y \times Y, \mathcal{B}_{F}:=Y \times Y \times X=\left(\pi_{F}\right)^{-1}(Y \times Y), \mathcal{B}_{S}:=$ $X \times Y \times Y=\left(\pi_{S}\right)^{-1}(Y \times Y)$, and $\mathcal{B}_{C}:=Y \times X \times Y=\left(\pi_{C}\right)^{-1}(Y \times Y)$. We define the triple b-stretched product, $X_{b}^{3}$, as the iterated blow-up, $X_{b}^{3}:=$ $\left[X^{3} ; \mathcal{T} ;\left\{\mathcal{B}_{F}, \mathcal{B}_{S}, \mathcal{B}_{C}\right\}\right]$. Figure 8 illustrates how $X_{b}^{3}$ is defined.

Let $\beta^{(3)}: X_{b}^{3} \longrightarrow X^{3}$ be the blow-down map. We define $l b:=\left(\beta^{(3)}\right)^{*}(Y \times$ $X \times X), r b:=\left(\beta^{(3)}\right)^{*}(X \times X \times Y), m b:=\left(\beta^{(3)}\right)^{*}(X \times Y \times X), f s:=$ $\left(\beta^{(3)}\right)^{*}(Y \times X \times X)$, ss $:=\left(\beta^{(3)}\right)^{*}(X \times X \times Y), c s:=\left(\beta^{(3)}\right)^{*}(X \times Y \times X)$, and $f f:=\left(\beta^{(3)}\right)^{*}(Y \times Y \times Y)$. (These faces are called the left boundary, right boundary, middle boundary, first side, second side, composite side, and front face respectively.) 


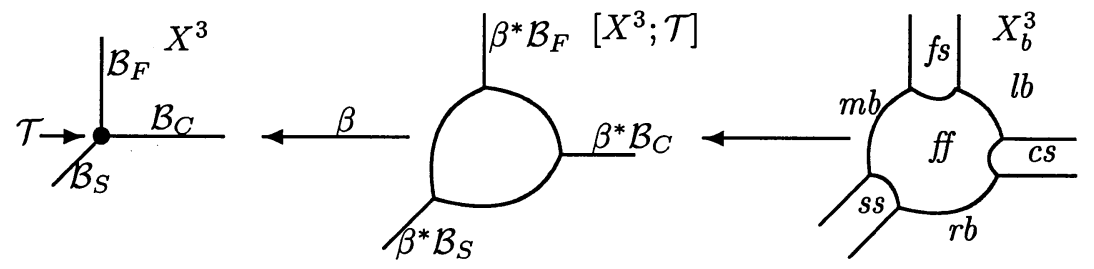

Figure 8: How $X_{b}^{3}$ is defined. The arrows represent blow-down maps.

Let $\beta: X_{b}^{2} \longrightarrow X^{2}$ be the blow-down map of $X_{b}^{2}$ onto $X^{2}$. Then, see [33], there exist (unique) functions $\pi_{O, b}: X_{b}^{3} \longrightarrow X_{b}^{2}$, where $O=F, S$, or $C$, such that

$$
\pi_{O} \circ \beta^{(3)}=\beta \circ \pi_{O, b} .
$$

Pictures of these functions are given in Figure 2, and these functions expressed in local coordinates are given in the proof of Lemma 4.3.

We now can show how to write the composition of $b$-pseudodifferential operators in terms of pullbacks and pushforwards. To do so, we first let $A_{1}, A_{2} \in \Psi_{b}^{-\infty}(X)$ have Schwartz kernels $K_{1}, K_{2}$ on $X^{2}$ vanishing to infinite order at $\partial X^{2}$. These are true smoothing operators. Then denoting the kernel of $A_{1} A_{2}$ on $X^{2}$ by $K_{12}$, observe that

$$
\left(\mu K_{12}\right)(x, z)=\mu(x) \int_{y \in X} K_{1}(x, y) K_{2}(y, z)=\left(\pi_{C}\right)_{*}\left(\pi_{C}^{*} \mu \pi_{F}^{*} K_{1} \pi_{S}^{*} K_{2}\right),
$$

where we continue to denote by $\mu$, the lift of $\mu$ to $X^{2}$ under $\pi_{L}$. Let $K_{A_{1}}=$ $\beta^{*} K_{1}$ and $K_{A_{2}}=\beta^{*} K_{2}$ be the kernels of $A_{1}$ and $A_{2}$ on $X_{b}^{2}$, and continuing to abuse notation, we denote $\beta^{*} \mu$ by $\mu$. Then by (1.6),

$$
\begin{aligned}
\mu K_{12} & =\left(\pi_{C}\right)_{*}\left(\pi_{C}^{*} \mu \pi_{F}^{*} K_{1} \pi_{S}^{*} K_{2}\right) \\
& =\left(\pi_{C}\right)_{*} \beta_{*}^{(3)}\left(\beta^{(3)}\right)^{*}\left(\pi_{C}^{*} \mu \pi_{F}^{*} K_{1} \pi_{S}^{*} K_{2}\right) \\
& =\beta_{*}\left(\pi_{C, b}\right)_{*}\left(\pi_{C, b}^{*} \beta^{*} \mu \pi_{F, b}^{*} \beta^{*} K_{1} \pi_{S, b}^{*} \beta^{*} K_{2}\right) \\
& =\beta_{*}\left(\pi_{C, b}\right)_{*}\left(\pi_{C, b}^{*} \mu \pi_{F, b}^{*} K_{A_{1}} \pi_{S, b}^{*} K_{A_{2}}\right) .
\end{aligned}
$$

Thus, the kernel of $\mu A_{1} A_{2}$ on $X_{b}^{2}$ is

$$
\mu K_{A_{1} A_{2}}=\left(\pi_{C, b}\right)_{*}\left(\pi_{C, b}^{*} \mu \pi_{F, b}^{*} K_{A_{2}} \pi_{S, b}^{*} K_{B_{2}}\right) .
$$

To derive this equation, we assumed that $A_{1}$ and $A_{2}$ were smoothing operators. But, using the properties of the maps $\pi_{C, b}, \pi_{F, b}, \pi_{S, b}$, and the pullback, product, and pushforward theorems of [14], one can show that 
this formula holds for $A_{1} \in \Psi_{b}^{m}(X)$ and $A_{2} \in \Psi_{b}^{m^{\prime}}(X)$, and moreover, that $A_{1} A_{2} \in \Psi_{b}^{m+m^{\prime}}(X)$. The proof uses Lemma 1.1 and is similar to, but much simpler than, the proof of Theorem 4.4.

\section{References.}

[1] S. Agmon, On the eigenfunctions and on the eigenvalues of general elliptic boundary value problems, Comm. Pure Appl. Math. 15 (1962), 119-147.

[2] S. Agmon and Y. Kannai, On the asymptotic behavior of spectral functions and resolvent kernels of elliptic operators, Israel J. Math. 5 (1967), $1-30$.

[3] N. Bleistein and R. A. Handelsman, Asymptotic expansions of integrals, Dover, New York, 1986.

[4] J. Brüning and R. Seeley, The expansion of the resolvent near a singular stratum of conical type, J. Funct. Anal. 95 (1991), no. 2, 255-290.

[5] - Regular singular asymptotics, Adv. in Math. 58 (1985), no. $2,133-148$.

[6] , The resolvent expansion for second order regular singular operators, J. Funct. Anal. 73 (1987), no. 2, 369-429.

[7] C. Callias, The heat equation with singular coefficients. I. Operators of the form $-d^{2} / d x^{2}+\kappa / x^{2}$ in dimension 1 , Comm. Math. Phys. 88 (1983), no. $3,357-385$.

[8] J. Cheeger, On the spectral geometry of spaces with cone-like singularities, Proc. Nat. Acad. Sci. U.S.A. 76 (1979), no. 5, 2103-2106.

[9] Spectral geometry of singular Riemannian spaces, J. Differential Geom. 18 (1983), no. 4, 575-657 (1984).

[10] J. Cheeger and M. Taylor, On the diffraction of waves by conical singularities. I, Comm. Pure Appl. Math. 35 (1982), no. 3, 275-331.

[11] A. Chou, The Dirac operator on spaces with conical singularities and positive scalar curvatures, Trans. Amer. Math. Soc. 289 (1985), no. 1, $1-40$.

[12] N. Dunford, Spectral theory, Bull. Amer. Math. Soc. 49 (1943), 637-651. 
[13] - Spectral theory. I. Convergence to projections, Trans. Amer. Math. Soc. 54 (1943), 185-217.

[14] C.L. Epstein, R.B. Melrose, and G.A. Mendoza, Resolvent of the laplacian on strictly pseudoconvex domains, Acta. Math. 167 (1991), 1-106.

[15] J.B. Gil, Full asymptotic expansion of the heat trace for non-self-adjoint elliptic cone operators, to appear in Math. Nachr.

[16] J.B. Gil and P. Loya, On the noncommutative residue and the heat trace expansion on conic manifolds, to appear in Manuscripta Math.

[17] P. Gilkey, Invariance theory, the heat equation, and the Atiyah-Singer index theorem, second ed., CRC Press, Boca Raton, FL, 1995.

[18] G. Grubb, Functional calculus of pseudodifferential boundary problems, second ed., Progress in Math., Birkhäuser, Boston, 1996.

[19] G. Grubb and R.T. Seeley, Weakly parametric pseudodifferential operators and Atiyah-Patoti-Singer operators, Invent. Math. 121 (1995), 481-529.

[20] L. Hörmander, On the Riesz means of spectral functions and eigenfunction expansions for elliptic differential operators, Some Recent Advances in the Basic Sciences, Vol. 2 (Proc. Annual Sci. Conf., Belfer Grad. School Sci., Yeshiva Univ., New York, 1965-1966), Belfer Graduate School of Science, Yeshiva Univ., New York, 1969, pp. 155-202.

[21] V. Ja. Ivriü, The second term of the spectral asymptotics for a LaplaceBeltrami operator on manifolds with boundary, Funktsional. Anal. i Prilozhen. 14 (1980), no. 2, 25-34.

[22] M. Kalka and A. Menikoff, The wave equation on a cone, Comm. Partial Differential Equations 7 (1982), no. 3, 223-278.

[23] A. I. Karol', Asymptotics of the parabolic Green function for an elliptic operator on a manifold with conical points, Mat. Zametki 63 (1998), no. $1,28-36$.

[24] Phạm The Lại and V. Petkov, Comportement asymptotique de la fonction spectrale de l'opérateur de Laplace-Beltrami sur une variété ayant des singularités coniques, Conference on Partial Differential Equations (Saint-Jean-de-Monts, 1983), Soc. Math. France, Paris, 1983, pp. Conf. No. $10,8$. 
[25] R. Lauter and J. Seiler, Pseudodifferential analysis on manifolds with boundary - a comparison of b-calculus and cone algebra, Approaches to singular analysis (Berlin, 1999), Birkhäuser, Basel, 2001, pp. 131-166.

[26] M. Lesch, Operators of Fuchs type, conical singularities, and asymptotic methods, B. G. Teubner Verlagsgesellschaft mbH, Stuttgart, 1997.

[27] P. Loya, Complex powers of differential operators on manifolds with conical singularities, to appear in J. Anal. Math.

[28] - Asymptotic properties of the heat kernel on conic manifolds, to appear in Israel J. of Math.

[29] $\longrightarrow$ The structure of the resolvent of elliptic pseudodifferential operators, J. Funct. Anal. 184 (2001), no. 1, 77-135.

[30] — Tempered operators and the heat kernel and complex powers of elliptic pseudodifferential operators, Comm. Partial Differential Equations 26 (2001), no. 7 \& 8, 1253-1321.

[31] R. Mazzeo, Elliptic theory of differential edge operators. I, Comm. Partial Differential Equations 16 (1991), no. 10, 1615-1664.

[32] R. McOwen, Pseudodifferential operators depending on a complex parameter, Global analysis-analysis on manifolds, Teubner, Leipzig, 1983, pp. 238-246.

[33] R.B. Melrose, Differential Analysis on Manifolds with Corners, in preparation.

[34] Calculus of conormal distributions on manifolds with corners, International Math. Research Notices 3 (1992), 51-61.

[35] - The Atiyah-Patodi-Singer Index Theorem, A.K. Peters, Wellesley, 1993.

[36] R.B. Melrose and V. Nistor, Homology of pseudodifferential operators I. Manifolds with boundary, preprint.

[37] R.B. Melrose and J. Wunsch, Singularities and the wave equation on conic spaces, National Research Symposium on Geometric Analysis and Applications (ANU, June 26-30, 2000), vol. 39, Australian National University, 2001. 
[38] E. Mooers, Heat kernel asymptotics on manifolds with conic singularities, J. Anal. Math. 78 (1999), 1-36.

[39] S. Rempel and B.-W. Schulze, Complex powers for pseudodifferential boundary problems. I, Math. Nachr. 111 (1983), 41-109.

[40] F. Riesz and Béla Sz.-Nagy, Functional analysis, Dover Publications Inc., New York, 1990, Translated from the second French edition by Leo F. Boron, Reprint of the 1955 original.

[41] E. Schrohe, Complex powers on noncompact manifolds and manifolds with singularities, Math. Ann. 281 (1988), no. 3, 393-409.

[42] — Noncommutative residues and manifolds with conical singularities, J. Funct. Anal. 150 (1997), no. 1, 146-174.

[43] B.-W. Schulze, Boundary value problems and singular pseudodifferential operators, J. Wiley, Chichester, 1998.

[44] R.T. Seeley, Complex powers of an elliptic operator, A.M.S. Symp. Pure Math. 10 (1967), 288-307.

[45] M. Shubin, Pseudodifferential operators and spectral theory, SpringerVerlag, Berlin, 1987, Translated from the Russian by Stig I. Andersson.

Department of Mathematics

BINGHAMTON UNIVERSITY

BINGHAMTON, NY 13902

USA

E-mail address: paul@math.binghamton.edu

ReCeived SePtember 5, 2000

REVISED NOVEMBER 28, 2001.

Supported by a Ford Foundation Fellowship administered by the National Research Council. 